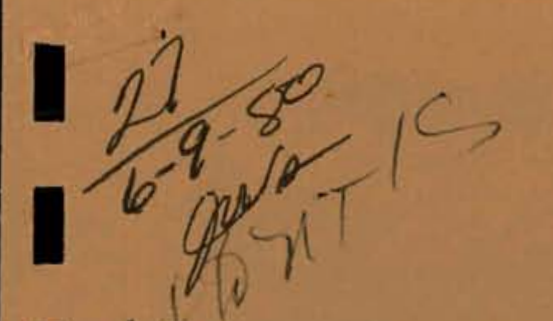

\title{
$\mathrm{DOE} / \mathrm{BC} / 10003-6$
}

\section{Western Gas Sands Project Status Report}

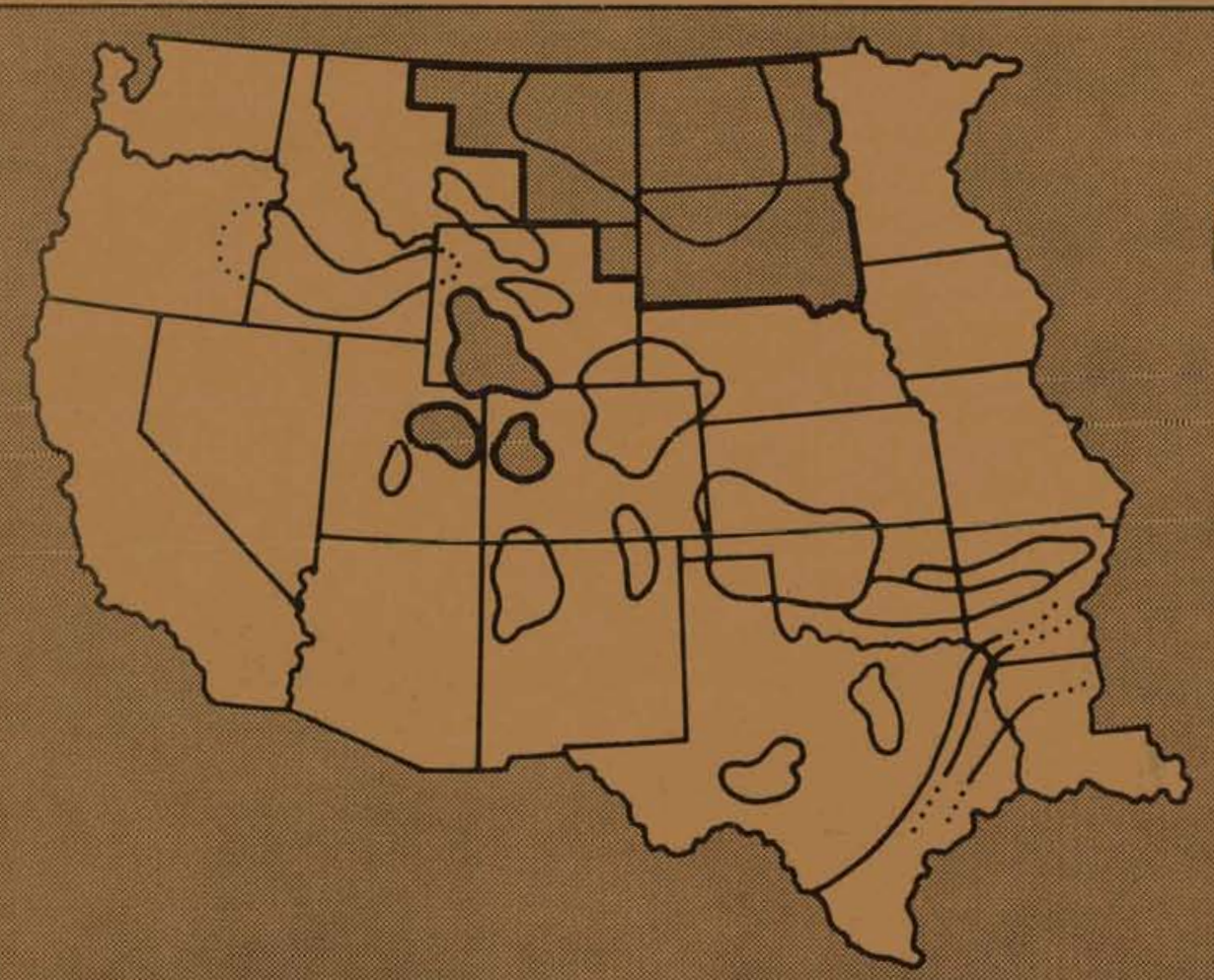

1 February - 29 February, 1980

Prepared for

U.S. Department of Energy

Bartlesville Energy Technology Center

Las Vegas Field Office

Compiled by CER Corporation

Las Vegas, Nevada

Contract DE-AC08-79BC10003 


\section{DISCLAIMER}

This report was prepared as an account of work sponsored by an agency of the United States Government. Neither the United States Government nor any agency Thereof, nor any of their employees, makes any warranty, express or implied, or assumes any legal liability or responsibility for the accuracy, completeness, or usefulness of any information, apparatus, product, or process disclosed, or represents that its use would not infringe privately owned rights. Reference herein to any specific commercial product, process, or service by trade name, trademark, manufacturer, or otherwise does not necessarily constitute or imply its endorsement, recommendation, or favoring by the United States Government or any agency thereof. The views and opinions of authors expressed herein do not necessarily state or reflect those of the United States Government or any agency thereof. 


\section{DISCLAIMER}

Portions of this document may be illegible in electronic image products. Images are produced from the best available original document. 
This report was prepared as an account of work sponsored by the United States Government. Neither the United States nor the United States Department of Energy, nor any of their employees, makes any warranty, express or implied, or assumes any legal liability or responsibility for the accuracy, completencss, or usefulness of any information, apparatus, product, or process disclosed, or represents that its use would not infringe privately owned rights. Reference herein to any specific commercial product, process, or service by trade name, mark, manufacturer, or otherwise, does not necessarily constitute or imply its endorsement, recommendation, or favoring by the United States Government or any agency thereof. The views and opinions of authors expressed herein do not necessarily state or reflect those of the United States Government or any agency thereof.

Available from the National Technical In formation Service, U. S. Department of Commerce, Springfield, Virginia 22161.

NATIONAL TECHNICAL INFORMATION SERVICE

PAPER COPY PRICES EFFECTIVE JANUARY 1,1979

$\begin{array}{lc}\begin{array}{c}\text { Page } \\ \text { Range }\end{array} & \begin{array}{c}\text { Domestic } \\ \text { Price }\end{array} \\ 001-025 & \$ 4.00 \\ 026-050 & \$ 4.50 \\ 051-075 & \$ 5.25 \\ 076-100 & \$ 6.00\end{array}$




\section{CONTENTS}

SECTION 1

SUMMARY

1

SECTION 2

PROJECT MANAGEMENT

- Technical Monitoring · 3

Technology Transfer 3

SECTION 3

RESOURCE ASSESSMENT . 7

U.S. Geological Survey Activities $\quad 7$

Core Program $\quad 8$

Survey of Basin Activities $\quad 8$

C K GeoEnergy Corporation $\quad 11$

Multi-Well Experiment $\quad 16$

SECTION 4

RESEARCH \& DEVELOPMENT BY

ENERGY TECHNOLOGY CENTERS

AND NATIONAL LABORATORIES

Bartlesville Energy Technology Center $\quad 17$

Gas Research Institute $\quad 23$

Lawrence Livermore Laboratory $\quad 26$

Los Alamos Scientific Laboratory $\quad 28$

Sandia Laboratories $\quad 31$

\section{SECTION 5}

FIELD TESTS AND DEMONSTRATIONS 39

Background $\quad 39$

Colorado Interstate Gas Company $\quad 42$

DOE Well Test Facility $\quad 44$

Gas Producing Enterprises, Inc. 50

Mitchell Energy Corporation $\quad 55$

Sandia Laboratories - Mineback $\quad 57$

This book was prepared as an accounc of wark sponsored by an agency of the United Stotes Government. warranty, express or implied, of ant nor any agency thereof, nor any of their employees, makes any completeness, or use? In ness of any intormation, apaaratus, product, or process discescouray. represents that its use would not intringe privately owned rights. Reterence herein to any specific commercial product, process, or service by trade name, trademark, manufacturer, or acherwise. dose 


\section{FIGURES}

Figure 3-1 Milestone Chart - USGS

Figure 3-2 Greater Green River Basin Showing

Wells of Interest and USGS Designated

Core Areas

Figure 3-3 Northern Great Plains Province Showing Wells of Interest and USGS Designated

Core Areas

Figure 3-4 Piceance Basin Showing Wells of Interest and USGS Designated Core Areas

Figure 3-5 Uinta Basin Showing Wells of Interest and USGS Designated Core Areas

Figure 3-6 Milestone Chart - Multi-Well

Experiment

Figure 4-1 Effect of Net Confining Pressure on Gas Slippage

Figure 4-2 Effect of Net Confining Pressure on Gas Slippage for Flow Through a Partially Saturated Core

Figure 4-3 Density Log Calibration Curve for CIGE Natural Buttes Unit No. 21 Well

Figure 4-4 Milestone Chart - BETC

Figure 4-5 Problem Geometry for Time Dependent Crack Propagation Near An Interface

Figure 4-6 Displacement Perpendicular to the Crack when $\mathrm{c} / \mathrm{L}=0.6$

Figure 4-7 Displacement Perpendicular to the Crack when $c / L=1.0$

Figure 4-8 Milestone Chart - LLL

Figure 4-9 Milestone Chart - LASL 


\section{FIGURES Continued}

Figure 4-10 Resistivity as a Function of Position Along the Probe for an Exponential Resisitivity Distribution

Figure 4-11 Milestone Chart - Sandia

Figure 5-1 Pressure and Flow Data from Miller No. 1 Drawdown Test

Figure 5-2 Miller No. 1 Pressure Buildup Data, January 30 - February 29, 1980

Figure 5-3 Flow Rate Performance of Natural Buttes No. 9 Well

Figure 5-4 Flow Rate Performance of Natural Buttes No. 14 Well

Figure 5-5 Flow Rate Performance of Natural Buttes No. 18 Well

Figure 5-6 Flow Rate Performance of Natural Buttes No. 19 Well

Figure 5-7 Flow Rate Performance of Natural Buttes No. 20 Well

Figure 5-8 Flow Rate Performance of Natural Buttes No. 22 Well

Figure 5-9 Experimental Coring Program of Hole No. 6 Fractures

Figure 5-10 Milestone Chart - Sandia Mineback 


\section{TABLES}

Table 3-1 Summary of Wells - Greater Green River Basin

Table 3-2 Summary of Wells - Northern Great Plains Province

Table 3-3 Summary of Wells - Piceance Basin

Table 3-4 Summary of Wells - Uinta Basin

Table 4-1 Summary of CEC Data

Table 4-2 Measurement of Net Confining Pressure on Gas Slippage

Table 4-3 Measurement of Net Confining Pressure on Gas Slippage Through a Partially Saturated Core

Table 4-4 Summary of Whole Core vs. Plug Analysis for CIGE No. 21

Table 4-5 Final Core Analysis Results for Eleven Samples from CIGE No. 21

Table 5-1 MHF Contract Location and Frac Data

Table 5-2 Miller No. 1 Drawdown Data, January 18 - 30, 1980

Table 5-3 Miller No. 1 Bottomhole Pressure Buildup Test, January 30 - February 29, 1980 


\section{SECTION 1}

\section{SUMMARY}

This edition of the WGSP Status Report summarizes the progress during February, 1980, of the governmentsponsored projects directed toward increasing gas production from low-permeability gas sands of the western United States. Background information on the project is provided in the WGSP Status Report, NVO/0655/100, September, 1977.

The National Laboratories and Energy Technology Centers continued research and experiments toward enhanced gas recovery. The field test and demonstration program continued with various projects, including test data collection by the DOE Well Test Facility at CIG's Miller No. 1 site. 
THIS PAGE

\section{WAS INTENTIONALLY \\ LEFT BLANK}




\section{SECTION 2}

\section{PROJECT MANAGEMENT}

\section{TECHNICAL MONITORING}

J. E. Evered (CER Corporation) attended the 1980 Energy-sources Technology Conference and Exhibition, February 3 through 7 in New Orleans, Louisiana. He presented a paper titled, "DOE/GRI Field Experiments in Western Low Permeability Reservoirs," co-authored by Bill Bailey (TRW). The conference was principally sponsored by the American Society of Mechanical Engineers.

C. H. Atkinson (DOE) and R. L. Mann (CER Corporation) met with C. L. Schuster and D. A. Northrop (Sandia Laboratories) in Las Vegas on February 13 to discuss the multi-well experiment plans and schedules.

C. H. Atkinson (DOE) met with A. Crawley (BETC) in Las Vegas, February 19 and 20 regarding the WGSP plans and budget.

J. C. Schillo (CER Corporation) observed pressure coring operations February 26-27, sponsored by Sandia Laboratories and performed on the Conoco Oil MCA No. 358 well, Lea County, New Mexico.

C. H. Atkinson (DOE) and R. L. Mann (CER Corporation) met with Northwest Exploration Company personnel in Denver, Colorado, on February 26 to discuss a possible North, west site for the WGSP multi-well experiment.

\section{TECHNOLOGY TRANSFER}

\section{Documentation Reports}

The WGSP Fourth Quarterly Basin Activities Report, 1979, and the WGSP Financial
Supplement for March, 1979, are being compiled. The WGSP Financial Supplement for February, 1979, has been distributed. The WGSP Logging Report is being finalized and the WGSP section of the DOE Semi-Annual Report of the Unconventional Gas: Recovery Program (period ending September, 1979) has been submitted to Morgantown. The DOE Well Test Facility Operations Manual and the WGSP Coring Program Document are being updated. Investigations continued toward a new WGSP Status Report format. Work continued on the WGSP Greater Green River Basin Review and similar geologic reports for the Piceance and Uinta Basins.

\section{Project Data Bank}

The following maps have been added to the WGSP data bank:

"Cross Section A-A' of Upper Cretaceous and Lower Tertiary Rocks, Northern Piceance Creek Basin, Colorado," by R. C. Johnson, U.S. Geological Survey MF-1129-A.

"Cross Section B-B' of Upper Cretaceous and Lower Tertiary Rocks, Northern Piceance Creek Basin, Colorado," by R. C. Johnson, U.S. Geological Survey MF1129-B.

"Cross Section C-C' of Upper Cretaceous and Lower Tertiary Rocks, Northern Piceance Creek Basin, Colorado," by R. C. Johnson, U.S. Geological Survey MF1129-C.

"Cross Section A-A' of Upper Cretaceous and Lower Tertiary Rocks, Southern Piceance Creek Basin, Colorado," by R. C. Johnson, M. P. Granica and N. C. Dessenberger, U.S. Geological Survey MF-1130-A.

"Cross Section B-B' of Upper Cretaceous and Lower Tertiary Rocks, Southern Piceance Creek Basin, Colorado," by R. C. Johnson, M. P. Granica and N. C. Dessenberger, U.S. Geological Survey MF1130-B. 
“Cross Section C-C' of Upper Cretaceous and Lower. Tertiary Rocks, Southern Piceance Creek'Basin, Colorado," by R. C. Johnson, M. P. Granica and N. C. Dessenberger, U.S. Geological Survey MF. 1130-C.

"Preliminary Chart Showing Distribution of Rock Types, Lithologic Groups and Depositional Environments for Some Early Tertiary and Late Cretaceous Rocks from Outcrops at Willow Creek-Indian Canyon through the Subsurface of Duchesne and Altamont Oil Fields, Southwest to North Central Parts of the Uinta Basin, Utah, Section A-A'," by T. D. Fouch, 1977.

"Preliminary Chart Showing Distribution of Rock Types, Lithologic Groups and Depositional Environments for Some Early Tertiary and Late Cretaceous Rocks from Outcrops at Willow Creek-Indian Canyon through the Subsurface of Duchesne and Altamont Oil Fields, Southwest to North Central Parts of the Uinta Basin, Utah, Section A'-A" ," by T. D. Fouch, 1977.

"Preliminary Chart Showing Distribution of Rock Types, Lithologic Groups and Depositional Environments for Some Lower Tertiary, Upper and Lower Cretaceous and Upper and Middle Jurassic Rocks in the Subsurface between Altamont Oil Field and San Arroyo Gas Field, Northcentral to Southeastern Uinta Basin, Utah, Section B' ," by T. D. Fouch and W. B. Cashion, 1979.

"Preliminary Chart Showing Distribution of Rock Types, Lithologic Groups and Depositional Environments for Some Lower Tertiary, Upper and Lower Cretaceous and Upper and Middle Jurassic Rocks in the Subsurface between Altamont Oil Field and San Arroyo Gas Field, Northcentral to Southeastern Uinta Basin, Utah, Section B"," by T. D. Fouch and W. B. Cashion, 1979.
Updating and cataloging continued on the WGSP bibiography and map file.

\section{Articles and Publications}

The following articles and publications relate to the WGSP and are included in the bibliography. Abstracts are included, when available. If a publication does not have an abstract, then the introduction is included.

\section{Characteristics of Pores in Sorne Upper Cretaceous Nonmarine Sandstones, Uinta Basin, Utah by C. W. Keighin USGS}

Porc characteristics of fine- to very fine-grained, nonmarine Upper Cretaceous sandstones in the Uinta Basin, Utah, appear to be modified less by mechanical compaction than by chemical processes. Dissolution of carbonates and some other mineral grains formed some intergranular pores having relatively smooth walls, but many of these pores are partially to extensively filled with kaolinite. Formation of authigenic kaolinite and illite produced much microporosity, with attendant high surface area and tortuous fluid-flow paths. These features have a strong influence on real or calculated water saturation, resistivity, sonic and neutron logs, and permeability.

Fourth Fracture Treatment Well No. 498-4-1, Rio Blanco

County, Colorado (Final Report)

by A. A. Ashteiwy and R. E. Chancellor

Van Poollen and Associates and Rio Blanco Natural Gas Co.

A moderate volume fracturing treatment was performed on a 600 -grossfoot section of the Mesa Verde/Fort Union in Rio Blanco Natural Gas Co. 
Well Federal No. 498-4-1. The treatment was designed to stimulate a multiple sand sequence at a minimum cost. The gross interval contains four separate sands with log calculated net thickness, porosities, and water saturations of 30 to 60 feet, 8.5 percent to 11.5 percent and 48 percent to 55 percent, respectively. After performing extensive remedial cementing and perforating, each individual zone was isolated and selectively broken down with acid, nitrogen and ball sealers. Commingled post-breakdown gas flow tested 100 to 130 MCFD. The frac treatment consisted of 143,000 gallons of gelled 2 percent $\mathrm{KCl}$ water, 44,000 pounds of 100 -mesh sand, 214,500 pounds of 20-40 mesh sand and 22,500 pounds of 12-20 mesh glass beads mixed with the sand as tail-in proppant. The well was fraced down tubing at 40 BPM and 2,300 to 3,900 psig. The post-frac tests indicate a rate of 350 MCFD and approximately 1 BPH of water, which is attributed to the middle two intervals. Water production from all zones commingled was relatively high; in excess of $10 \mathrm{BPH}$. Water entry is believed to be through the perfs in the uppermost sand and the water source is possibly from some water-bearing sands up the hole. 


\section{THIS PAGE \\ WAS INTENTIONALLY \\ LEFT BLANK}




\section{SECTION 3}

\section{RESOURCE ASSESSMENT}

\section{U.S. GEOLOGICAL SURVEY ACTIVITIES}

USGS Open-file reports may be ordered from:

Open-file Services Section (OFSS)

Branch of Distribution

U.S. Geological Survey

- Box 25425, MS 306

Federal Center

Lakewood, Colorado 80225

\section{Uinta-Piceance Basins}

Structure (top of Cretaceous) and isopach interval maps of some Upper Cretaceous rocks in the Piceance Basin were submitted for review prior to publication.

The geologic appropriateness of a proposed DOE multi-well test and core site in the Piceance Basin near the Divide Creek Anticline was reviewed. The analysis indicated that some gas-bearing Upper Cretaceous rocks would be encountered. However, the proposed location is far enough away from subsurface control points to be a wildcat test and near enough to surface exposures of the target rocks to perhaps contain a number of water-bearing reservoirs. The analysis was based on calculation of borehole log properties, analysis of drillstem test results and knowledge of the stratigraphy and depositional character of the Upper Cretaceous rocks in the region.

Manuscripts are being prepared that describe and discuss rocks in the producing intervals at Pariette Bench and Southman Canyon fields in the Uinta Basin.
A stratigraphic section is being prepared that relates rock types, producing intervals, and formation and engineering data for Upper Cretaceous and lower Tertiary rocks extending from Price River Canyon to the eastern Uinta Basin.

U.S. Geological Survey Open-File Report 79-1702 was published: "Preliminary results of mineralogic and diagenetic studies of low-permeability sandstones of Late Cretaceous age, Piceance Creek Basin, Northwestern Colorado," by P. L. Hansley and R. C. Johnson, 1980.

\section{Greater Green River Basin}

Work continued on a stratigraphic analysis of the Greater Green River Basin.

Gas desorption was completed on core samples from Rainbow Resources No. 1-3 Pacific Creek Federal well, Sec. 3, T26N, R103W, Sweetwater County, Wyoming.

A manuscript was begun by B. E. Law concerning the correlation of Upper Cretaceous rocks between the Hoback Basin and Rock Springs Uplift, Wyoming.

C. W. Spencer prepared "Bibliography of publications prepared by the U.S. Geological Survey for the U.S. Department of Energy's Western Gas Sands Project from April 1977, to December 1979." Copies are available from CFR Corporation, Las Vegas, Nevada.

The following maps were published:

Tyler, T. F., and Peterson, J. R., 1980, "Wildcat well penetration map showing wells drilled into and through potentially gas-bearing, low-permeability Upper Cretaceous and Tertiary reservoirs, Washakie Basin, Wyoming," U.S. Geological Survey Open-File Report 80-189, 1 sheet. 
Tyler, T. F., 1980, "Preliminary chart showing electric-log correlation section E-E' of some Upper Cretaceous and Tertiary rocks, Cherokee Ridge, Wyoming," U.S. Geological Survey OpenFile Report 80-191, 4 sheets.

\section{Northern Great Plains Province}

D. D. Rice continued work on a regional cross section from southeastern Alberta to northcentral Montana.

A report entitled "Generation, Accumulation, and Resource Potential of Biogenic Gas," by D. D. Rice and G. E. Claypool was revised for the AAPG Bulletin.

D. L. Gautier is preparing an open-file report on the regional reservoir characteristics of the Eagle Sandstone and equivalent rocks.

\section{Schedule Status}

Figure $3-1$ is a milestone chart showing USGS progress within the WGSP.

\section{CORE PROGRAM}

Coring plans were suspended for Midlands Gas No. 2 Federal 2670 well, Sec. 26, T37N, R30E, Phillips County, Montana. Drilling was delayed and the well has temporarily been taken off the active list. However, it may be re-activated at a later date.

Several operators were contacted during the month in regards to the WGSP coring program. Among them were Cotton Petroleum and CIGE concerning wells in the Washakie Basin (Greater Green River Basin), and American Resources Management in the Piceance Basin.

\section{SURVEY OF BASIN ACTIVITIES}

\section{Greater Green River Basin}

During February, 15 wells were reported complete: 3 development wells were producers, 7 wildcat wells were discoveries and 5 wildcat tests were D\&A. (One wildcat location was temporarily abandoned and will be used as a non-commercial research well.) Initial potential flow for the 10 producing wells totaled 11,785 MCFD. The Almond Formation was the largest contributor $(4,827$ MCFD), with the Lewis, Ericson, Frontier and Mesaverde undifferentiated also contributing.

Newly staked locations totaled 29: 22 development and 7 wildcat.

Table $3-1$ is a summary of wells in the Greater Green River Basin, and Figure 3-2 shows their locations.

\section{Northern Greát Plains Province}

Nineteen wells were reported complete during the month: 9 development wells were producers, 2 wildcat wells were discuveries, and 3 development and 5 wildcat wells were D\&A.

From the 11 producing wells, the initial potential flow totaled 4,927 MCFD. The producing horizons included the Greenhorn, Eagle, Fish Scales, White Specks, Bow Island and Ferdig/Blackleaf/Bow Island commingled.

Seven new locations were staked during February: 4 development and 3 wildcat.

A summary of wells located in the province is in Table 3-2, and Figure 3-3 shows their locations. 


\begin{tabular}{|c|c|c|c|c|c|c|c|c|c|c|c|c|c|}
\hline \multicolumn{2}{|r|}{ WGSP - USGS } & \multicolumn{12}{|c|}{ FY-80 } \\
\hline A. & Greater Green River Basin & OCT & Nov & DEC & JAN & FEB & MAR & APR & MAY & JUN & JUL & AUG & SEP \\
\hline \multicolumn{14}{|c|}{ Continue studies of stratigraphy. } \\
\hline \multicolumn{14}{|c|}{ Prepare and open-file two electric-log } \\
\hline \multicolumn{14}{|c|}{ Prepare and open-file one electric-log } \\
\hline \multicolumn{14}{|c|}{ Complete cross section network in } \\
\hline \multicolumn{14}{|c|}{ END } \\
\hline$\nabla$ & $\begin{array}{l}\text { Scheduled Start and Completion } \\
\text { of Task }\end{array}$ & & & & & & & & & & & & \\
\hline & Projected Schedule & & & & & & & & & & & & \\
\hline$\equiv$ & Task Progress Not Reported & & & & & & & & & & & & \\
\hline 20001 & Delay in Work on Task & & & & & & & & & & & & \\
\hline
\end{tabular}

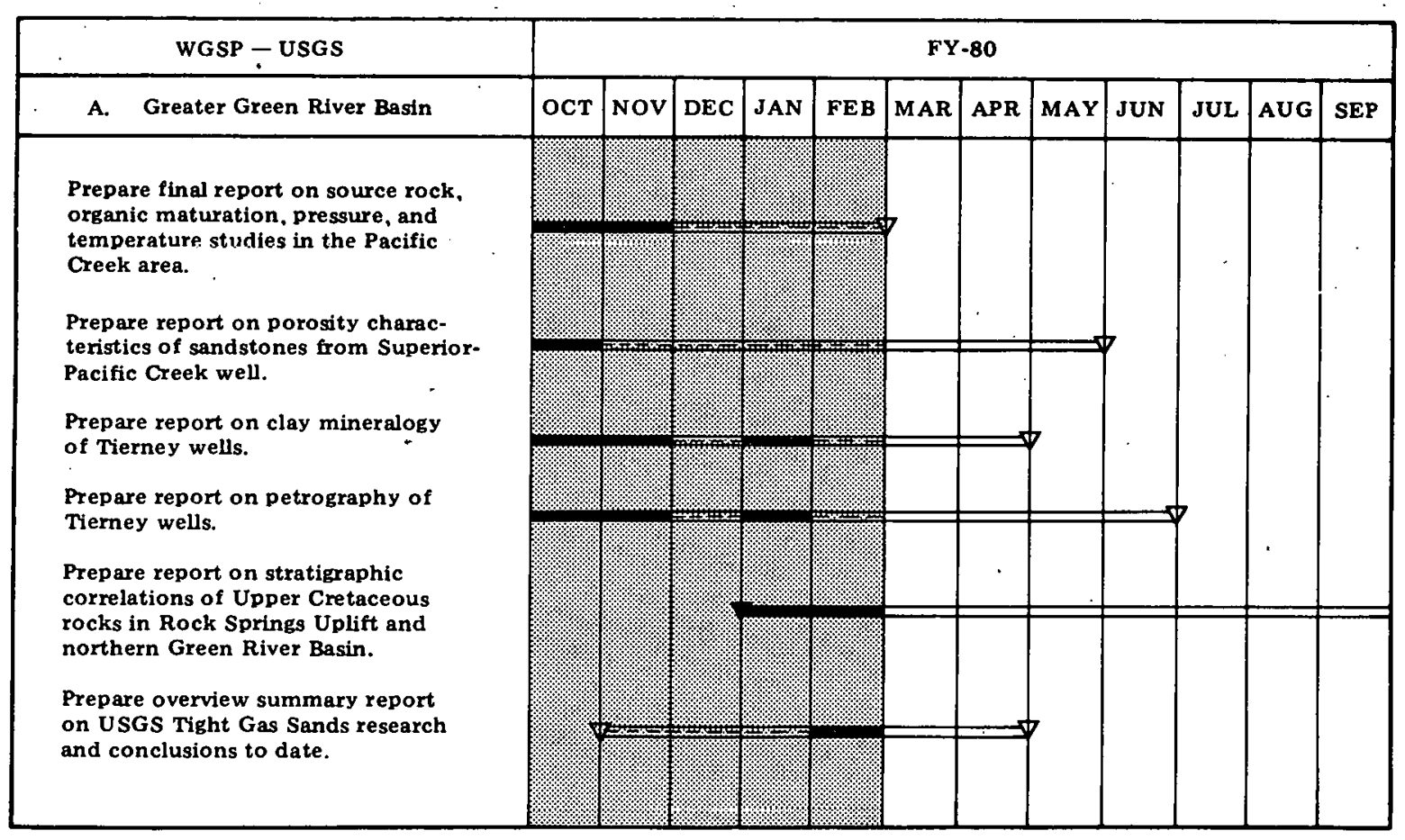

Figure 3-1 Milestone Chart-USGS 


\begin{tabular}{|c|c|c|c|c|c|c|c|c|c|c|c|c|}
\hline WGSP - USGS & \multicolumn{12}{|c|}{ FY -80} \\
\hline B. Northern Great Plains Province & OCT & Nov & DEC & JAN & FEB & MAR & APR & MAY & JUN & JUL & AUG & SEP \\
\hline \multicolumn{13}{|l|}{$\begin{array}{l}\text { Petrographic examination of producing } \\
\text { low-permeabillty reservoirs of SE } \\
\text { Alberta. }\end{array}$} \\
\hline \multicolumn{13}{|l|}{$\begin{array}{l}\text { Write paper on origin of biogenic gas } \\
\text { accumulations. }\end{array}$} \\
\hline \multicolumn{13}{|l|}{$\begin{array}{l}\text { Construct cross-sections from } \\
\text { western Canada to north-central } \\
\text { Montana. }\end{array}$} \\
\hline \multicolumn{13}{|l|}{$\begin{array}{l}\text { Write paper on depositional environ- } \\
\text { ments and geometries of Mosby SS } \\
\text { (Greenhorn Fm.) of north-central } \\
\text { Montana. }\end{array}$} \\
\hline \multicolumn{13}{|l|}{$\begin{array}{l}\text { Regional subsurface study of Niobrara } \\
\mathrm{Fm} \text {. in North and South Dakota. }\end{array}$} \\
\hline \multicolumn{13}{|l|}{ Pilot lineament study of Northerm } \\
\hline Black Hills. & & & & & & & & & & & & \\
\hline \multicolumn{13}{|l|}{ Subsurface study of Groat sandstone. } \\
\hline Regional lineament map. & & . & 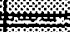 & 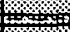 & 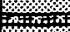 & & & & & & & \\
\hline & & & & & & & & & & & & \\
\hline
\end{tabular}

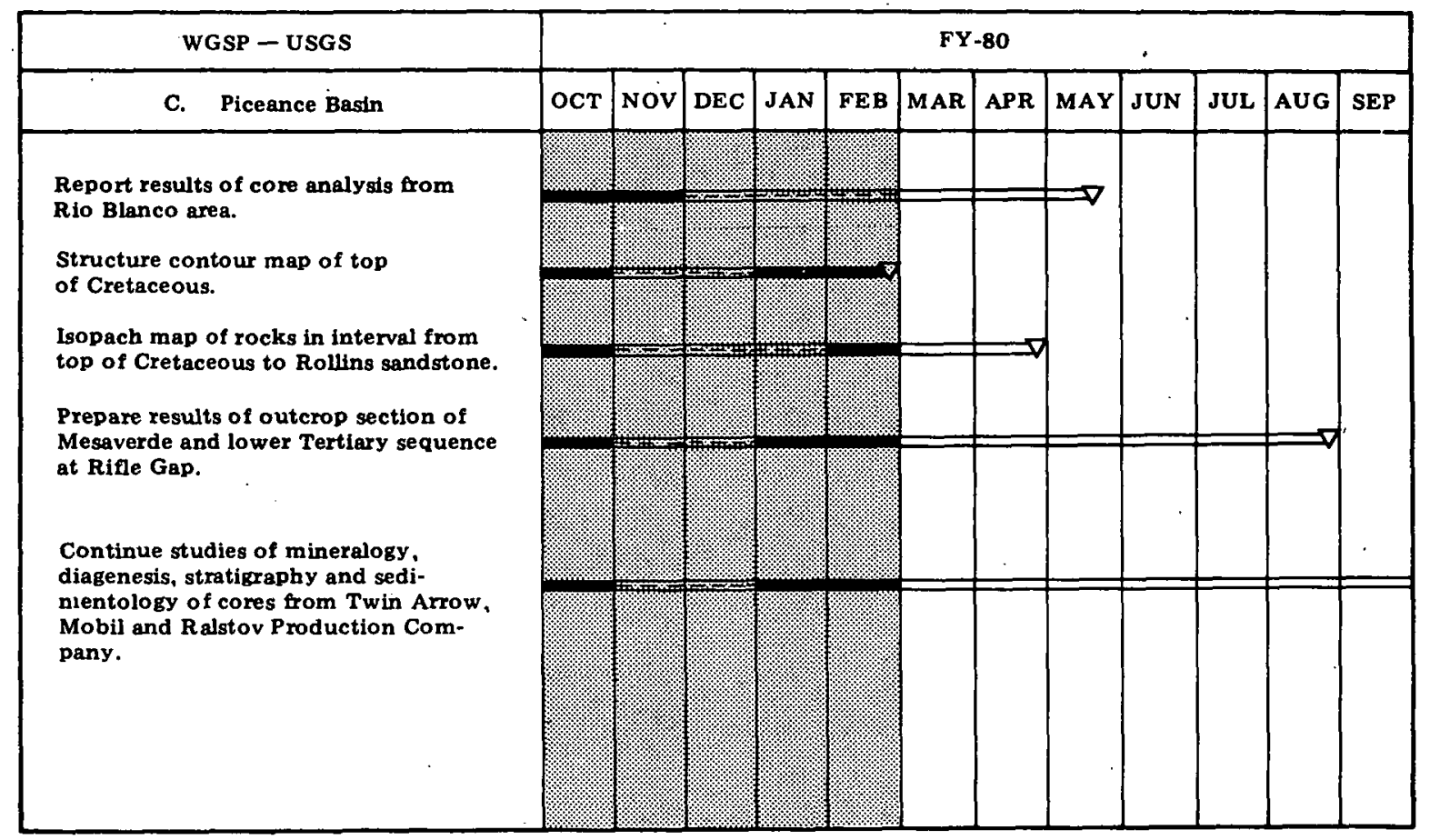

Figure 3-1 Continued 


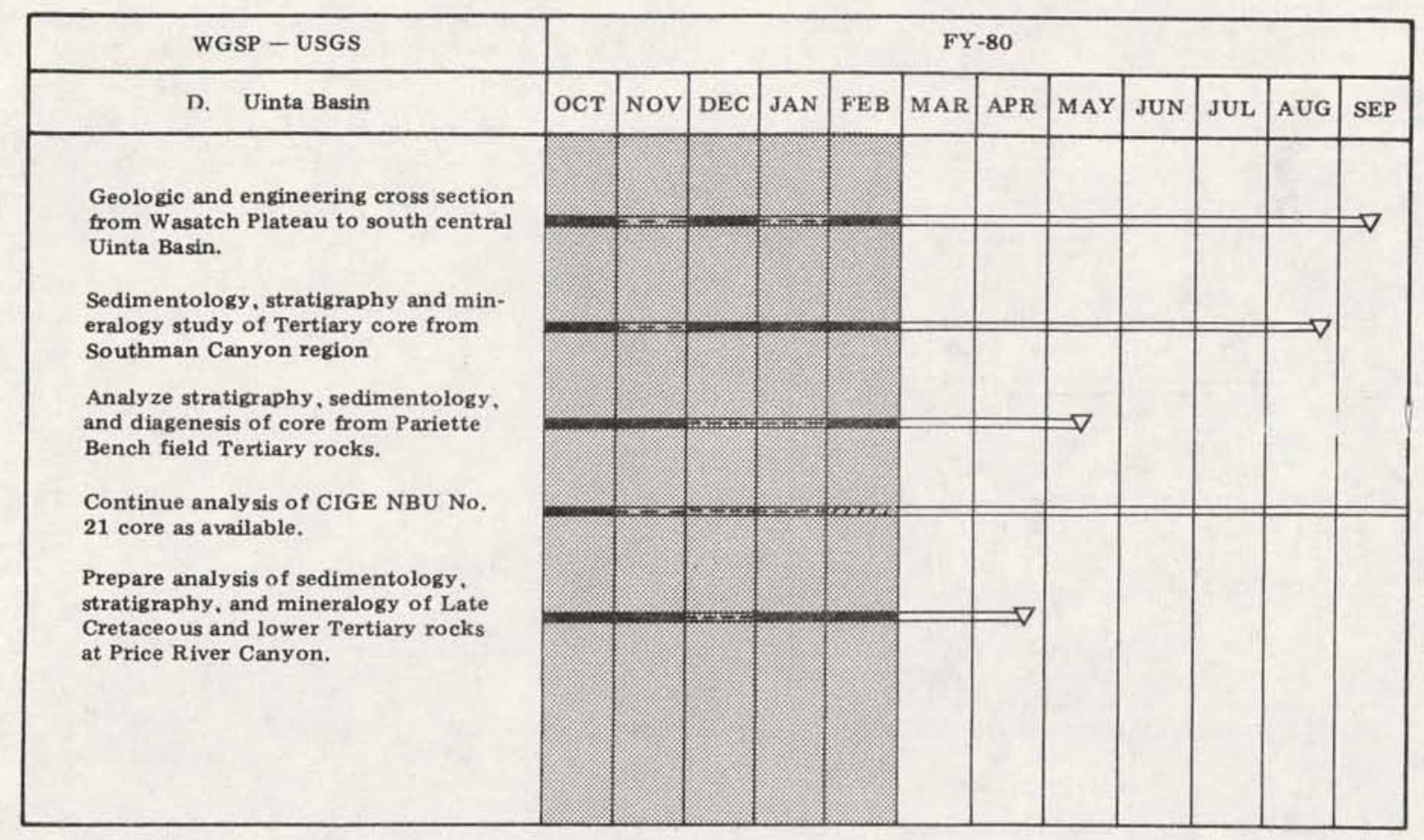

Figure 3-1 Continued

\section{Piceance Basin}

During the month, 10 wells were reported complete: 8 development wells were producers, 1 wildcat well was a discovery and 1 development well was D\&A.

Total initial potential flow was 4,083 MCFD. The Mancos B was the largest contributor with 3,234 MCFD (1 IPF was not available for a shut-in gas well). Other horizons included the Cozzette/Corcoran commingled and Morapos/Mancos B commingled.

Five development and four wildcat locations were staked by month's end.

Table 3-3 is a summary of wells located in the Piceance Basin, and Figure 3-4 shows their locations.

\section{Uinta Basin}

Seven wells were reported complete this month: 6 development wells were producers and 1 wildcat well was a discovery. Initial potential flow totaled 8,032 MCFD, with the Wasatch and Mesaverde undifferentiated as contributors. The Wasatch/Mesaverde commingled contributed the largest amount of new gas: 4,408 MCFD.

No new locations were staked during the month.

A summary of wells for the basin is in Table $3-4$, and the wells' locations are shown in Figure 3-5.

\section{K GEOENERGY}

Processing of field data continued for contract No. DE-AC08-79BC10005 (Development of Techniques for Optimizing Selection and Completion of Western Tight Gas Sands). Final reports are in progress. With CK GeoEnergy's assistance, Schlumberger is interested in following up the work that has been started. 


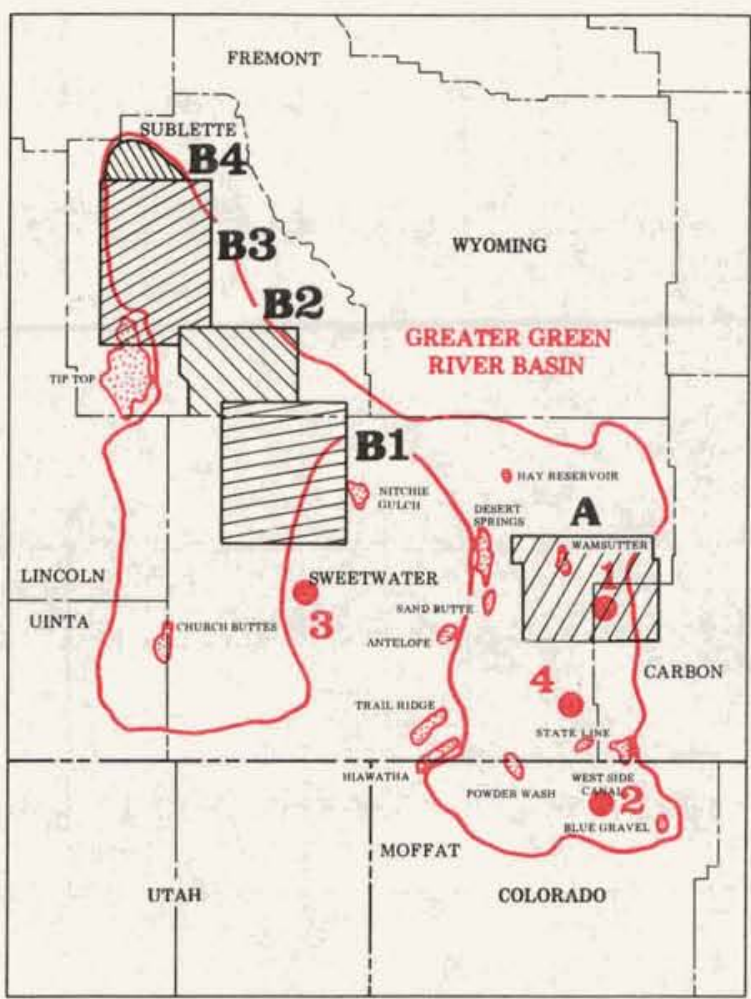

Figure 3-2 Greater Green River Basin Showing Wells of Interest and USGS Designated Core Areas (refer to Table 3-1)

Table 3-1 Summary of Wells - Greater Green River Basin

\begin{tabular}{|c|c|c|c|c|c|c|c|c|}
\hline OPERATOR & $\begin{array}{l}\text { WELL } \\
\text { NAME }\end{array}$ & $\begin{array}{l}\text { MAP } \\
\text { INDEX } \\
\text { NO. }^{1}\end{array}$ & $\begin{array}{l}\text { LOCATION } \\
\text { Sec/T/R }\end{array}$ & $\underset{\mathrm{ft}}{\text { HORIZON }}$ & $\begin{array}{c}\text { FIN AL } \\
\text { TD }\end{array}$ & $\begin{array}{l}\text { FRACTURE } \\
\text { TREATMENT }\end{array}$ & STATUS & $\begin{array}{l}\text { IPF in } \\
\text { MCFD }\end{array}$ \\
\hline $\begin{array}{l}\text { Amoco } \\
\text { Production }\end{array}$ & $\begin{array}{l}1 \text { Champlin } \\
226 \text { Amoco- } \\
\text { G }\end{array}$ & 1 & $\begin{array}{l}\text { csw } 33 / 18 \mathrm{~N} / 93 \mathrm{~W} \\
\text { Unnamed Field } \\
\text { Carbon Cnty, WY }\end{array}$ & $\begin{array}{l}\text { Almond } \\
8,918-9,094 \\
\text { Ericson } \\
9,124-9,204 \\
\text { (commingled) }\end{array}$ & 9,307 & $\begin{array}{ll}202,230 & \text { gal } \\
\text { gel, } 366,300 & \text { e } \\
\text { lb sand. } & 1 \\
& \text { ir }\end{array}$ & $\begin{array}{l}\text { Wildcat outpost } \\
\text { extension, comp. } \\
12-5-79 . \text { No cores } \\
\text { or tests. Located } \\
\text { in Core Area A. }\end{array}$ & 4,900 \\
\hline $\begin{array}{l}\text { Tenneco } \\
\text { Oil }\end{array}$ & 1-10 Pilgrim & 2 & $\begin{array}{l}\text { csw } 10 / 10 N / 93 w \\
\text { Unnamed Field } \\
\text { Moffat Cnty, Co }\end{array}$ & $\begin{array}{l}\text { Lewis } \\
7,438-7,456\end{array}$ & $\begin{array}{l}9,450 \\
\text { PB: } \\
8,895\end{array}$ & $\begin{array}{l}\text { Acidized w/ } \\
4,000 \text { gal; } \\
\text { fractured w/ } \\
82,000 \text { gal } \\
\text { acid, } 77,052 \\
\text { gal water, } \\
24,000 \text { gal } \\
\text { gel, } 260,500 \\
\text { lb sand. }\end{array}$ & $\begin{array}{l}\text { Wildeat field } \\
\text { discovery, comp. } \\
\text { 12-12-79. }\end{array}$ & 425 \\
\hline Davis Oil & $\begin{array}{l}1 \text { Sue- } \\
\text { Federal }\end{array}$ & 3 & $\begin{array}{l}\text { cne } 32 / 19 \mathrm{~N} / 106 \mathrm{~W} \\
\text { Unnamed Field } \\
\text { Sweetwater Cnty, } \\
\text { WY }\end{array}$ & $\begin{array}{l}\text { Frontier } \\
14,653-14,704\end{array}$ & $\begin{array}{l}15,445 \\
\text { PB: } \\
15,406\end{array}$ & $\begin{array}{l}\text { Acidized w/ } \\
8,000 \mathrm{gal} ; \\
\text { fractured w/ } \\
147,000 \mathrm{gal} \\
\text { water, } 169,000 \\
\text { lb sand. }\end{array}$ & $\begin{array}{l}\text { Wildcat field } \\
\text { discovery, comp. } \\
\text { 5-16-79. No tests. } \\
0\end{array}$ & 118 \\
\hline Getty Oil & $\begin{array}{l}23-2 \\
\text { McPherson } \\
\text { Springs }\end{array}$ & 4 & $\begin{array}{l}\text { nwne } 23 / 13 N / 94 W \\
\text { Unnamed Field } \\
\text { Sweetwater Cnty. } \\
\text { WY }\end{array}$ & $\begin{array}{l}\text { Lewis } \\
10,219-11,010\end{array}$ & $\begin{array}{l}12,131 \\
\text { PB: } \\
11,036\end{array}$ & $\begin{array}{l}\text { Acidized w/ } \\
7,500 \mathrm{gal} ; \\
\text { fractured w/ } \\
40,400 \mathrm{gal} \\
\text { water, } 14,700 \\
\text { lb sand. }\end{array}$ & $\begin{array}{l}\text { Wildcat field } \\
\text { discovery, comp. } \\
1-2-80 \text {. No cores. }\end{array}$ & 990 \\
\hline
\end{tabular}

1Refer to Figure 3-2

${ }^{2}$ Horizon - projected depth or producing interval 


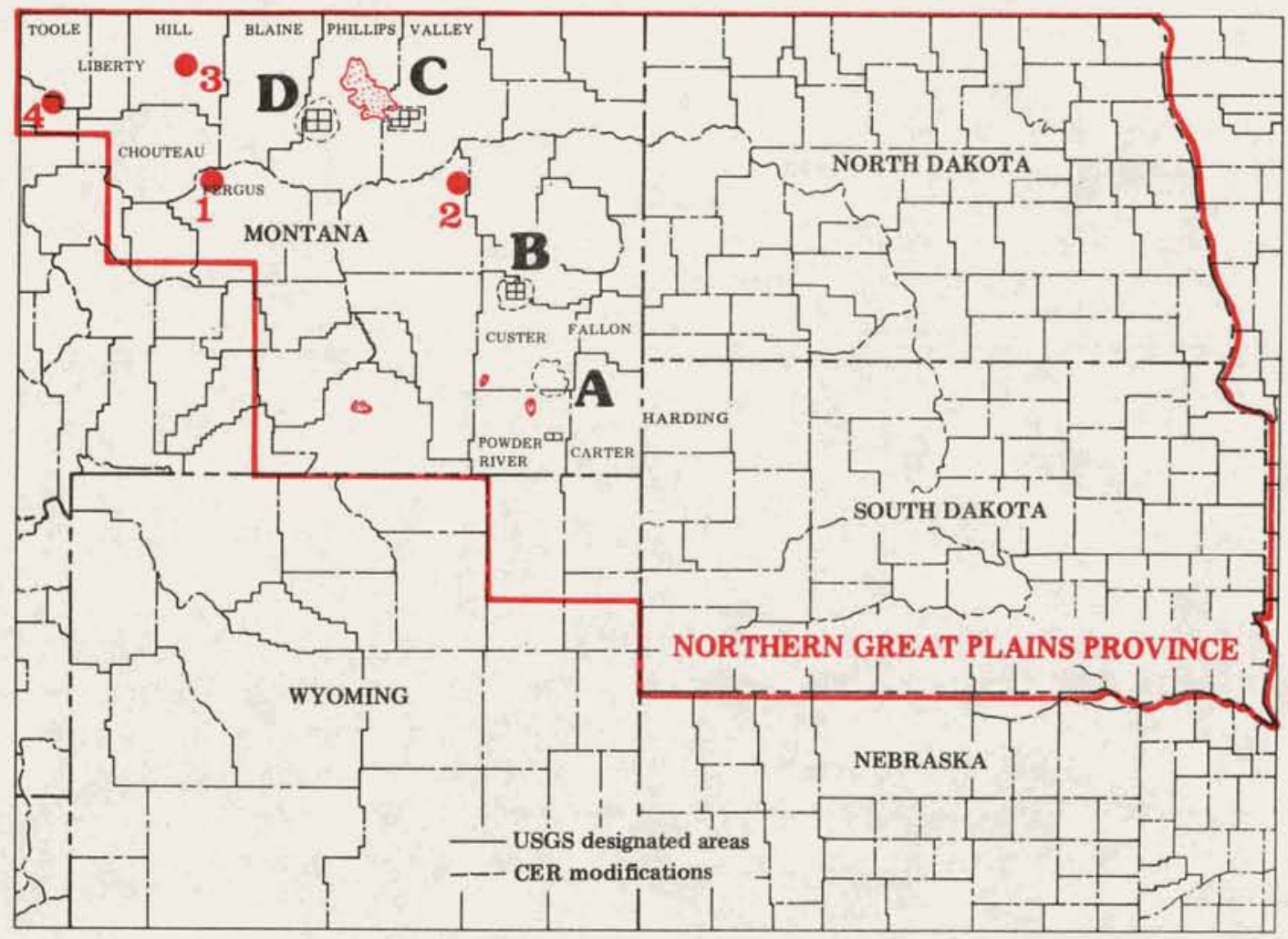

Figure 3-3 Northern Great Plains Province Showing Wells of Interest and USGS Designated Core Areas (refer to Table 3-2)

Table 3-2 Summary of Wells - Northern Great Plains Province

\begin{tabular}{|c|c|c|c|c|c|c|c|c|}
\hline OPERATOR & $\begin{array}{l}\text { WELL } \\
\text { NAME }\end{array}$ & $\begin{array}{c}\text { MAP } \\
\text { RDEX } \\
\text { NO. }\end{array}$ & $\begin{array}{l}\text { LOCATION } \\
\text { Sec/T/R }\end{array}$ & $\underset{\mathrm{ft}}{\text { HORIZON }}$ & $\begin{array}{c}\text { FINAL } \\
\text { TD }\end{array}$ & $\begin{array}{l}\text { FRACTURE } \\
\text { TREATMENT }\end{array}$ & STATUS & $\begin{array}{l}\text { IPF in } \\
\text { MCFD }\end{array}$ \\
\hline $\begin{array}{l}\text { South Ranch } \\
\text { Oil }\end{array}$ & 1 Dickson & 1 & $\begin{array}{l}\text { sese } 9 / 21 \mathrm{~N} / 15 \mathrm{E} \\
\text { Wildcat Field } \\
\text { Fergus Cnty, MT }\end{array}$ & $\begin{array}{l}\text { Muddy } \\
2,387\end{array}$ & 2,469 & & $\begin{array}{l}\text { Wildcat test, comp. } \\
1-27-80 \text { D\&A. } \\
\text { Drill stem tested; } \\
\text { no cores. }\end{array}$ & \\
\hline Gulf Oil & $\begin{array}{l}1-19 \\
\text { Federal }\end{array}$ & 2 & $\begin{array}{l}\text { nese } 19 / 21 \mathrm{~N} / 42 \mathrm{E} \\
\text { Wildeat Field } \\
\text { Garfield Cnty, MT }\end{array}$ & $\begin{array}{l}\text { Eagle } \\
2,450\end{array}$ & 2,050 & & $\begin{array}{l}\text { Wildcat test, comp. } \\
11-30-79 \text { D\& A. } \\
\text { No cores or tests. }\end{array}$ & \\
\hline $\begin{array}{l}\text { Charles } \\
\text { W. Austin }\end{array}$ & $\begin{array}{l}15-7 \\
\text { Woeppel }\end{array}$ & 3 & $\begin{array}{l}\text { swne } 15 / 34 \mathrm{~N} / 12 \mathrm{E} \\
\text { Unnamed Field } \\
\text { Hill Cnty, MT }\end{array}$ & $\begin{array}{l}\text { Eagle } \\
1,008-1,010\end{array}$ & 1,307 & & $\begin{array}{l}\text { Wildcat field } \\
\text { discovery, comp. } \\
8-22-79 \text {. No cores } \\
\text { or tests. }\end{array}$ & 227 \\
\hline $\begin{array}{l}\text { Balcron } \\
\text { Oil }\end{array}$ & $\begin{array}{l}\text { 1-32 } \\
\text { Sprague }\end{array}$ & 4 & $\begin{array}{l}\text { swne } 32 / 30 \mathrm{~N} / 2 \mathrm{~W} \\
\text { Wildcat Field } \\
\text { Pondera Cnty, MT }\end{array}$ & $\begin{array}{l}\text { Bow Island } \\
1,400\end{array}$ & & & $\begin{array}{l}\text { Wildcat test, } \\
\text { reported 2-20-80. }\end{array}$ & \\
\hline
\end{tabular}

1 Refer to Figure 3-3

${ }^{2}$ Horizon - projected depth

or producing interval 


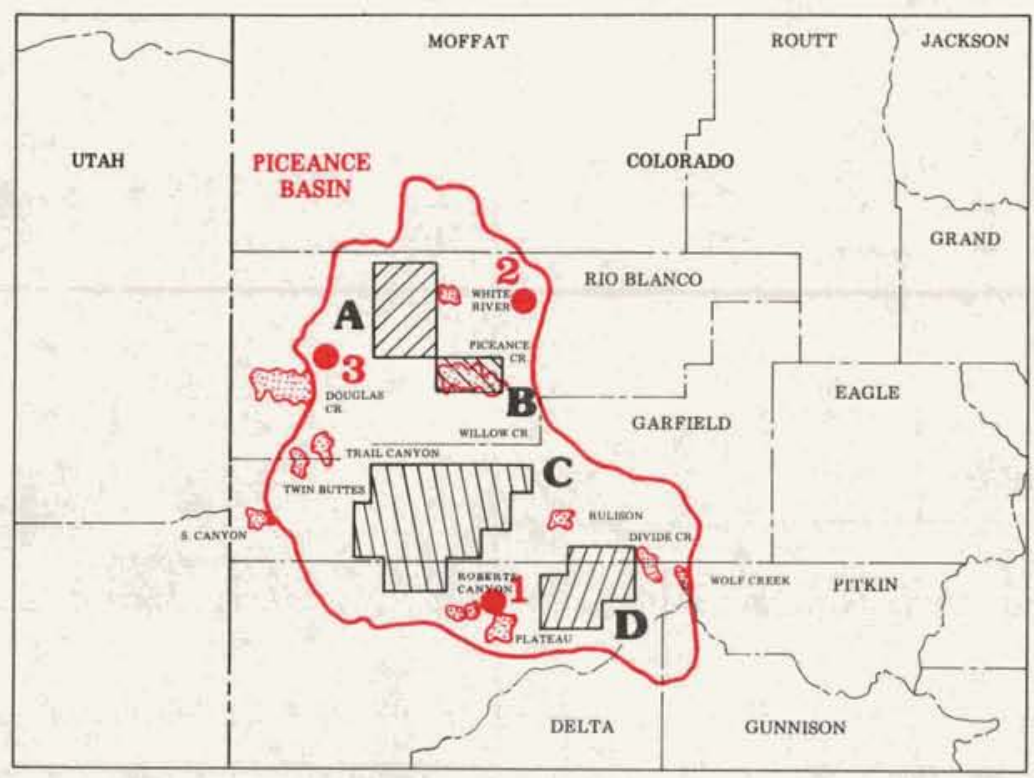

Figure 3-4 Piceance Basin Showing Wells of Interest and USGS Designated Core Areas (refer to Table 3-3)

Table 3-3 Summary of Wells - Piceance Basin

\begin{tabular}{|c|c|c|c|c|c|c|c|c|}
\hline OPERATOR & $\begin{array}{l}\text { WELL } \\
\text { NAME }\end{array}$ & $\begin{array}{l}\text { MAP } \\
\text { INDEX } \\
\text { NO. }\end{array}$ & $\begin{array}{l}\text { LOCATION } \\
\text { Sec/T/R }\end{array}$ & $\underset{f t}{\text { HORIZON }}{ }^{2}$ & $\begin{array}{l}\text { FINAL } \\
\text { TD }\end{array}$ & $\begin{array}{l}\text { FRACTURE } \\
\text { TREATMENT }\end{array}$ & STATUS & $\begin{array}{l}\text { IPF in } \\
\text { MCFD }\end{array}$ \\
\hline $\begin{array}{l}\text { Chandler } \\
\text { \& Associates }\end{array}$ & $\begin{array}{l}\text { 11-32 } \\
\text { North } \\
\text { Plateau } \\
\text { Creek }\end{array}$ & 1 & $\begin{array}{l}\text { nesw } 32 / 9 S / 96 W \\
\text { Shire Guleh Field } \\
\text { Mesa Cnty, CO }\end{array}$ & $\begin{array}{l}\text { Cozzette } \\
3,918-4,064 \\
\text { Corcoran } \\
4,065-4,125 \\
\text { (commingled) }\end{array}$ & $\begin{array}{l}4,323 \\
\text { PB: } \\
4,089\end{array}$ & $\begin{array}{l}\text { Acidized w/ } \\
4,200 \mathrm{gal} ; \\
\text { fractured w/ } \\
\mathbf{6 6 , 9 5 6} \mathrm{gal} \\
\text { fluid, } 261,000 \\
\text { lb sand. }\end{array}$ & $\begin{array}{l}\text { Develop. well, } \\
\text { comp. 12-20-79. } \\
\text { Cored; no tests. } \\
\end{array}$ & 440 \\
\hline $\begin{array}{l}\text { Northwest } \\
\text { Exploration }\end{array}$ & $\begin{array}{l}1 \text { HD } \\
\text { Lake }\end{array}$ & 2 & $\begin{array}{l}\text { nese } 22 / 1 \mathrm{~N} / 95 \mathrm{~W} \\
\text { Powell Park Field } \\
\text { Rio Blanco Cnty, } \\
\text { CO }\end{array}$ & $\begin{array}{l}\text { Mancos B } \\
12,320-12,450 \\
\text { (gross) }\end{array}$ & $\begin{array}{l}12,704 \\
\text { PB: } \\
12,508\end{array}$ & $\begin{array}{l}50,000 \mathrm{gal} \\
\text { emul, } \\
60,000 \mathrm{lb} \\
\text { sand. }\end{array}$ & $\begin{array}{l}\text { Wildcat deeper } \\
\text { pool discovery, } \\
\text { comp. } 7-9-79 . \\
\text { No cores or tests. } \\
\text { OWWO, comp. } \\
10-31-75 ; \text { prod. } \\
\text { zone: Mesaverde. }\end{array}$ & 322 \\
\hline $\begin{array}{l}\text { Chandler } \\
\text { \& Associates }\end{array}$ & $\begin{array}{l}12-29-1-1 \\
\text { Fork Unit }\end{array}$ & 3 & $\begin{array}{l}\text { nesw } 29 / 1 \text { S/101W } \\
\text { Dragon Trail Field } \\
\text { Rio Blanco Cnty, } \\
\text { CO }\end{array}$ & $\begin{array}{l}\text { Morapos } \\
1,083-1,101 \\
\text { Mancos B } \\
2,430-2,495 \\
\text { (commingled) }\end{array}$ & 2,835 & $\begin{array}{l}\text { Acidized } \\
\text { thrice w/ } \\
\text { total } 3,250 \\
\text { gal; fractured } \\
\text { twice w/total } \\
142,800 \text { gal } \\
\text { fluid, } 363,000 \\
\text { lb sand. }\end{array}$ & $\begin{array}{l}\text { Develop. well, } \\
\text { comp. 12-10-79. } \\
\text { No cores or tests. }\end{array}$ & 409 \\
\hline
\end{tabular}

${ }^{1}$ Refer to Figure 3-4

${ }^{2}$ Horizon - projected depth

or producing interval 


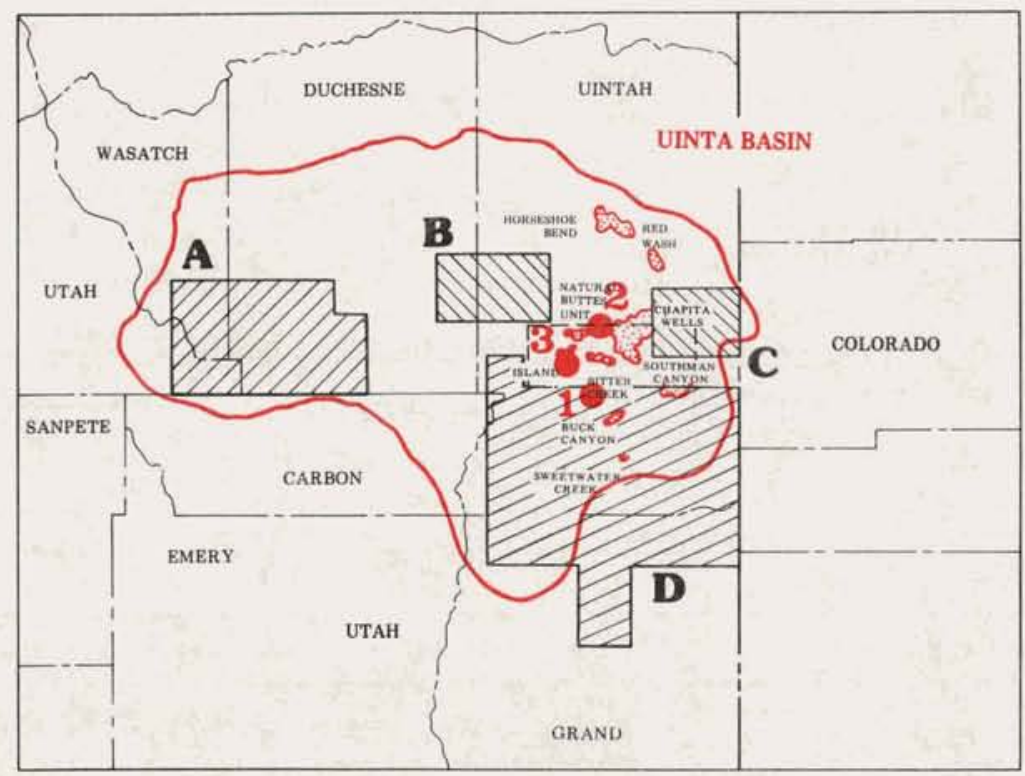

Figure 3-5 Uinta Basin Showing Wells of Interest and USGS Designated Core Areas (refer to Table 3-4)

Table 3-4 Summary of Wells - Uinta Basin

\begin{tabular}{|c|c|c|c|c|c|c|c|c|}
\hline OPERATOR & $\begin{array}{l}\text { WELL } \\
\text { NAME }\end{array}$ & $\begin{array}{l}\text { MAP } \\
\text { INDEX } \\
\text { NO. }\end{array}$ & $\begin{array}{l}\text { LOCATION } \\
\text { Sec/T/R }\end{array}$ & $\underset{\mathrm{ft}}{\text { HORIZON }}$ & $\begin{array}{c}\text { FINAL } \\
\text { TD }\end{array}$ & $\begin{array}{l}\text { FRACTURE } \\
\text { TREATMENT }\end{array}$ & STATUS & $\begin{array}{l}\text { IPF in } \\
\text { MCFD }\end{array}$ \\
\hline $\begin{array}{l}\text { Cotton } \\
\text { Petroleum }\end{array}$ & $\begin{array}{l}1 \text { Love } \\
\text { Unit }\end{array}$ & 1 & $\begin{array}{l}\text { csw } 11 / 11 \mathrm{~S} / 21 \mathrm{E} \\
\text { Unnamed Field } \\
\text { Uintah Cnty, UT }\end{array}$ & $\begin{array}{l}\text { Mesaverde } \\
6,659-7,734 \\
\text { (gross) }\end{array}$ & $\begin{array}{l}8,069 \\
\text { PB: } \\
7,592\end{array}$ & $\begin{array}{l}\text { Fractured } \\
\text { twice w/ } \\
\text { total } \\
267,700 \mathrm{gal} \\
\text { fluid, } \\
609,725 \mathrm{lb} \\
\text { sand. }\end{array}$ & $\begin{array}{l}\text { Wildcat field } \\
\text { discovery, comp. } \\
1-19-80 \text {. No } \\
\text { cores or tests. } \\
\text { Located in } \\
\text { Core Area D. }\end{array}$ & 1,122 \\
\hline Gulf Oil & $\begin{array}{l}1-36 \\
\text { Morgan- } \\
\text { State }\end{array}$ & 2 & $\begin{array}{l}\text { senw } 36 / 9 S / 21 \mathrm{E} \\
\text { Natural Buttes } \\
\text { Field } \\
\text { Uintah Cnty, UT }\end{array}$ & $\begin{array}{l}\text { Mesaverde } \\
6,962-9,272 \\
\text { (gross) }\end{array}$ & 9,531 & $\begin{array}{l}16,580 \mathrm{gal} \\
\text { fluid, } \\
240,000 \mathrm{lb} \\
\text { sand. }\end{array}$ & $\begin{array}{l}\text { Develop. well, } \\
\text { comp. } 2-2-80 \text {. } \\
\text { No cores or tests. }\end{array}$ & 870 \\
\hline $\begin{array}{l}\text { Mapco } \\
\text { Production }\end{array}$ & $\begin{array}{l}\text { 7-11-F } \\
\text { River Bend } \\
\text { Unit }\end{array}$ & d & $\begin{array}{l}\text { swne } 11 / 10 S / 20 \mathrm{E} \\
\text { River Bend Field } \\
\text { Uintah Cnty, UT }\end{array}$ & $\begin{array}{l}\text { Wasatch } \\
5,946-6,619 \\
\text { (gross) }\end{array}$ & $\begin{array}{l}8,691 \\
\text { PB: } \\
8,647\end{array}$ & $\begin{array}{l}68,500 \text { gal } \\
\text { gel, } 150,000 \\
\text { lb sand. }\end{array}$ & $\begin{array}{l}\text { Develop. well, } \\
\text { comp. 1-7-80. } \\
\text { No cores or tests. }\end{array}$ & 1,632 \\
\hline
\end{tabular}

\footnotetext{
${ }^{1}$ Refer to Figure 3-5

2 Horizon - projected depth

or producing interval
} 
A final report is in preparation for contract No. ET-78C-08-1601 (Reservoir Characteristics in Uinta Basin Gas Wells).

\section{MULTI-WELL EXPERIMENT}

The selection of a site for the experiment has not been finalized. However, after investigation of a number of potential sites, the selection was narrowed to three locations.

After the geology and offset well data were evaluated, a Northwest Exploration site near Rifle, Colorado, appeared to be the most suitable. The final selection will be made by the Multi-Well Advisory Committee in March.

Figure $3-6$ is a milestone chart showing the progress of the multi-well experiment.

\begin{tabular}{|c|c|c|c|c|c|c|c|c|c|c|c|c|c|}
\hline & WGSP & & & & & & FY & -80 & & & & & \\
\hline MU & LTI-WELL EXPERIMENT & OCT & Nov & DEC & JAN & FEB & MAR & APR & MAY & JUN & JUL & AUG & SEP \\
\hline Plar & ning Activities & & & & & & & & & & & & \\
\hline Site & Acquisition and Permits & & & & & & & & & & & & \\
\hline Site & Preparation & & & & & & & & & & & & \\
\hline Dril & ing, Coring, Logging & & & & & & & & & & & $\Rightarrow \nabla$ & \\
\hline Wel & Testing & & & & & & & & & & & & \\
\hline Cor & Analyses & & & & & & & & & & & & \\
\hline LEGEND & & & & & & & & & & & & & \\
\hline$\nabla$ & $\begin{array}{l}\text { Scheduled Start and Completion } \\
\text { of Task }\end{array}$ & & & & & & & & & & & & \\
\hline$\nabla$ & $\begin{array}{l}\text { Completed Milestone } \\
\text { Projected Schedule }\end{array}$ & & & & & & & & & & & & \\
\hline$=$ & Task Progressing & & & & & & & & & & & & \\
\hline$\overline{10=11}$ & Task Progress Not Reported & & & & & & & & & & & & \\
\hline Ееке & & & & & & & & & & & & & \\
\hline
\end{tabular}

Figure 3-6 Milestone Chart-Multi-Well Experiment 


\section{SECTION 4}

\section{RESEARCH \& DEVELOPMENT BY ENERGY TECHNOLOGY CENTERS \& NATIONAL LABORATORIES}

\section{BARTLESVILLE ENERGY TECHNOLOGY CENTER}

\section{Improved Pressure Coring System - Sandia Laboratories}

\section{Core Retriever Design and Test}

After a pressure core has been cut, it must be separated from the formation by creating a tensile/shear fracture at the lower end of the core. Due to the large cutting radius on the inner gauge surface of a natural diamond bit, the core usually breaks at a point several inches above the bottom of the hole. Past core barrels have been designed for this type of breakage. When Stratapax ${ }^{\circledR}$ bits are used, the core breaks much closer to the bottom of the hole because the bit cuts a smaller core to formation radius. This type of face design is necessary to reduce fluid invasion of the core. In a few instances, the resulting longer core has prevented proper closure of the ball valve mechanism. To solve this problem, several components of the pressure coring system are being redesigned to permit increased downward travel of the ball valve. This will eliminate clearance difficulty.

\section{Core Fluid Tests}

The control unit for the large capacity air free mixer has been received and is being installed.
Testing should be completed in March with field trials set for April. $\mathrm{CaBr} 2$ has been substituted for the $\mathrm{CaCl}_{2}$ brine base in recent laboratory tests. The filtration characteristics appear unchanged while the fluid does not freeze at dry ice temperatures. This has the potential for a further simplification in the preparation of cores for analysis. Additional laboratory and field tests are planned.

\section{Bit Design and Fabrication}

The new Sandia designed core bit bodies are scheduled to be completed in March. Steel studs with diffusion-bonded PDC cutters will also be available for bit assembly in March. Plans to diffusion bond PDC cutters to the pilot bit bodies are being formulated.

\section{Polycrystalline Diamond Core Bit Tests}

Requests for PDC core bits continue for pressure coring operations by the major oil companies.

\section{Logging Studies}

Interface Conductivity Effects on Electric Logging

Evaluation of the core cation exchange capacity (CEC) from five wells with in the WGSP area has been completed. The intervals ranged from 500 to $10,000 \mathrm{ft}$ in depth. The CEC of core samples from the J. J. C. Paine No. 1-0296 well, Northern Great Plains Province, was generally much higher than for the other wells (see Table 4-1). X-ray mineral analysis of samples from the J. J. C. Paine well indicated higher amounts of montmorillonite. Shales in the remaining wells contained very little montmorillonite and derived their CEC primarily from illite. The CEC of a particular sample was the average of two to seven determinations. The mean error of the CEC 
Table 4-1 Summary of CEC Data

\begin{tabular}{|c|c|c|c|c|c|}
\hline Well & Location & $\begin{array}{c}\text { Core Interval } \\
(\mathrm{ft})\end{array}$ & $\begin{array}{l}\text { CEC Range } \\
\text { (meq/g) }\end{array}$ & $\begin{array}{l}\text { No. of } \\
\text { Samples } \\
\text { (n) }\end{array}$ & $\begin{array}{l}\text { Mean Factor } \\
\text { of CEC Values }\end{array}$ \\
\hline $\begin{array}{l}\text { Mobil } \\
\text { PCU 31-13 }\end{array}$ & Piceance Basin & $8,490-10,000$ & $0.021-0.034$ & 4 & $\begin{array}{r} \pm 0.001 \\
( \pm 3.3 \%)\end{array}$ \\
\hline $\begin{array}{l}\text { CIGE } \\
21-15-10-22\end{array}$ & Uinta Basin & $4,460-7,430$ & $0.009-0.061$ & 8 & $\begin{array}{r} \pm 0.001 \\
( \pm 4.4 \%)\end{array}$ \\
\hline $\begin{array}{l}\text { J. J. C. Paine } \\
\text { 1-0296 Federal }\end{array}$ & $\begin{array}{l}\text { Northern Great } \\
\text { Plains Pruvince }\end{array}$ & $550-1,680$ & $0.049-0.302$ & 16 & $\begin{array}{r} \pm 0.002 \\
( \pm 1.1 \%)\end{array}$ \\
\hline $\begin{array}{l}\text { Pacific Transmission } \\
\text { Supply } \\
24-19 \text { Federal }\end{array}$ & $\begin{array}{l}\text { Greater Green } \\
\text { River Basin }\end{array}$ & $5,120-5,220$ & $0.006 \cdot 0.142$ & 14 & $\begin{array}{r} \pm 0.001 \\
( \pm 6.6 \%)\end{array}$ \\
\hline $\begin{array}{l}\text { Twin Arrow } \\
\text { C\&K 4-14: }\end{array}$ & Piceance Basin & $990-2,000$ & $0.004-0.138$ & 22 & $\begin{array}{r} \pm 0.001 \\
( \pm 4.3 \%)\end{array}$ \\
\hline
\end{tabular}

values for each well is included in Table 4-1, along with the percentage mean error (in parenthesis).

Mapping and Contouring Water Resistivities in Tight Western Gas Sands - Texas A\&M University

Of the 22 Uinta Basin wells from which reliable water samples were available, 13 electric logs were found in the Denver library of Petroleum Information Corporation. Of these logs, one was from the Mesaverde Group (Castlegate Formation), nine from the Green River Formation and three from the Pennsylvanian-Weber Formation.

The analysis of the Castlegate water and the calculation of formation water resistivity $\left(R_{W}\right)$ are as follows:

Well Location: Sec. 27, T9S, R24E, Uintah County, Utah

Depth of Perforations: 7,194 to $7,299 \mathrm{ft}$

Resistivity of Formation Water: .52 ohm-meter @ 680 $\mathrm{F}$

\begin{tabular}{lccr}
\hline Ion & Concentration, ppm & Multiplier & Equivalent NaCl \\
\hline $\mathrm{Na}$ & 5,178 & 1.00 & 5,178 \\
$\mathrm{Ca}$ & 63 & 0.95 & 60 \\
$\mathrm{Mg}$ & 17 & 2.00 & 34 \\
$\mathrm{SO}_{4}$ & 480 & 0.50 & 240 \\
$\mathrm{Cl}$ & 7,000 & 1.00 & 7,000 \\
$\mathrm{HCO}$ & 1,366 & 0.27 & 369 \\
& & & 12,880 \\
\hline
\end{tabular}

Calculated $\mathrm{R}_{\mathrm{W}}: 0.51$ ohm-meter@680 $\mathrm{F}$

Calculated $\mathrm{R}_{\mathrm{W}}: 0.23$ ohm-meter@1500F

Calculated mud-filtrate resistivity $\left(R_{\mathrm{mf}}\right): 1.80 \mathrm{ohm}-$ meter $@ 150^{\circ} \mathrm{F}$ 
The maximum value of the self potential (SP) appears to be -30 millivolts.

Applying the relationship:

$$
\mathrm{SP}=-\left(60+0.133 \mathrm{~T}{ }^{\circ} \mathrm{F}\right) \log _{10} \frac{\mathrm{R}_{\mathrm{mf}}}{\mathrm{R}_{\mathrm{W}}}
$$

in which the term in parenthesis is the temperature relationship, the value of $R_{W}$ is calculated to be $0.75 \mathrm{ohm}$-meters. In order for the above relationship to yield an $R_{W}$ of 0.23 ohm-meters, the SP would have to be -71 millivolts.

It is well established that as the content of fine material in a porous matrix is increased, the absolute value of the self potential is decreased in magnitude. This behavior was utilized by Knutson and Boardman 1 in their differentiation of fluvial and lacustrine formations. Therefore, it appears likely that this particular formation is so filled with fines, even in the section of highest porosity, the self potential has been more than halved.

Although the Green River Formation is not a target western tight gas sand, the available water analyses and electric logs were compared. In all cases, the self potential was similarly depressed with respect to the recorded value of $R_{W}$.

It appears that a more reliable form of the relationship between self potential and resistivities would be:

$$
\mathrm{SP}=-\mathrm{K}(460+\mathrm{T}) \log 10 \frac{\mathrm{R}_{\mathrm{mf}}}{\mathbf{R}_{\mathrm{W}}}
$$

where $\mathbf{T}=$ degrees fahrenheit.

An equation of this form will be developed when more formation water samples become available. Contacts with operators in the Uinta Basin are being sought in an effort to obtain a library of reliable formation waters from producing wells in the Upper Cretaceous formations.

\footnotetext{
1 Knutson, C. F. and C. R. Boardman, "Continuity and Permeability Development in the Tight Gas Sands of the Eastern Uinta Basin."
}

\section{In Situ Permeability}

Measurement of Formation Characteristics for Western Tight Sands - Institute of Gas Technology

Measurements have commenced on cores from the Mapco RBU No. 11-17F well, Sec. 17, T10S, R20E, Uintah County, Utah. The results of measurements on core No. 8298.8 are shown in Figure 4-1. Gas permeabilities were measured as a function of pore pressure under different confining pressures. It was observed that the slope of the line relating gas permeability and reciprocal mean pore pressure decreased with increasing net confining pressure, as did the permeability value at infinite pore pressure. The slope intercept and the Klinkenberg constant $b$ are shown in Table 4-2. The slope decreased from 1.675 (md)(psi) at a net confining pressure of 120 psi to $0.319(\mathrm{md})(\mathrm{psi})$ at a net confining pressure of $5,000 \mathrm{psi}$. The slope intercept decreased from $0.346 \mathrm{md}$ to $0.0124 \mathrm{md}$. The values of $b$ did not exhibit any trend.

Irvestigation of the pore-pressure dependence of permeability for the Mobil PCU No. F3113G well core No. 9998A, indicated that the. slope of the Kinkenberg plot decreased with increasing net confining pressure for a partially water saturated core $\left(\mathrm{S}_{\mathrm{W}}=20.9\right.$ percent). The slope decreased from 0.608 (md) (psi) at a net confining pressure of 120 psi to 0.119 (md)(psi) at 3,000 psi net confining pressure. The intercept decreased more rapidly, from 0.0101 md at 120 psi net confining pressure to 0.0007 md at a net confining pressure of 3,000 psi. The results are shown in Figure 4-2 and Table 4-3.

\section{Advanced Logging Techniques and Interpretations}

Developments and Applications of a Wide Band Laboratory Dielectric Constant Measurement System - Colorado School of Mines 


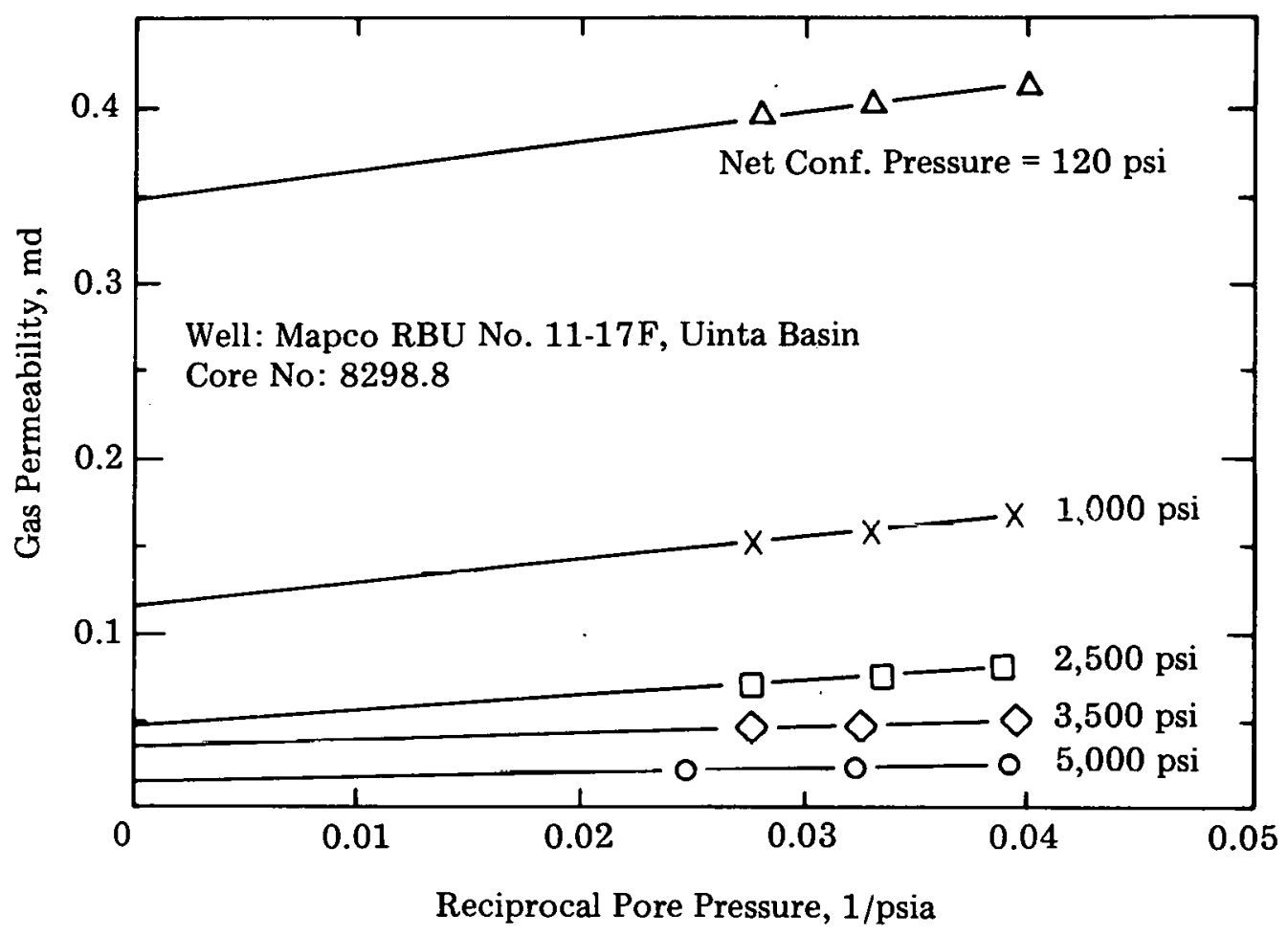

Figure 4-1 Effect of Net Confining Pressure on Gas Slippage

Table 4-2 Measurement of Net Confining Pressure on Gas Slippage

Well: Mapco RBU No. 11-17F, Uinta Basin

Core No: $\mathbf{8 2 9 8 . 8}$

\begin{tabular}{cccc}
\hline $\begin{array}{l}\text { Net Confining } \\
\text { Pressure, psi }\end{array}$ & $\begin{array}{c}\text { Slope } \\
(\mathbf{m d})(\mathbf{p s i})\end{array}$ & $\begin{array}{r}\text { Intercept } \\
\mathbf{K} \infty, \mathbf{m d}\end{array}$ & $\begin{array}{r}\mathbf{b}, \\
\mathbf{a t m}\end{array}$ \\
\hline 120 & 1.675 & 0.3460 & 0.33 \\
1,000 & 1.290 & 0.1160 & 0.75 \\
2,500 & 0.868 & 0.0467 & 1.26 \\
3,500 & 0.368 & 0.0358 & 0.70 \\
5,000 & 0.319 & 0.0124 & 1.74 \\
\hline
\end{tabular}




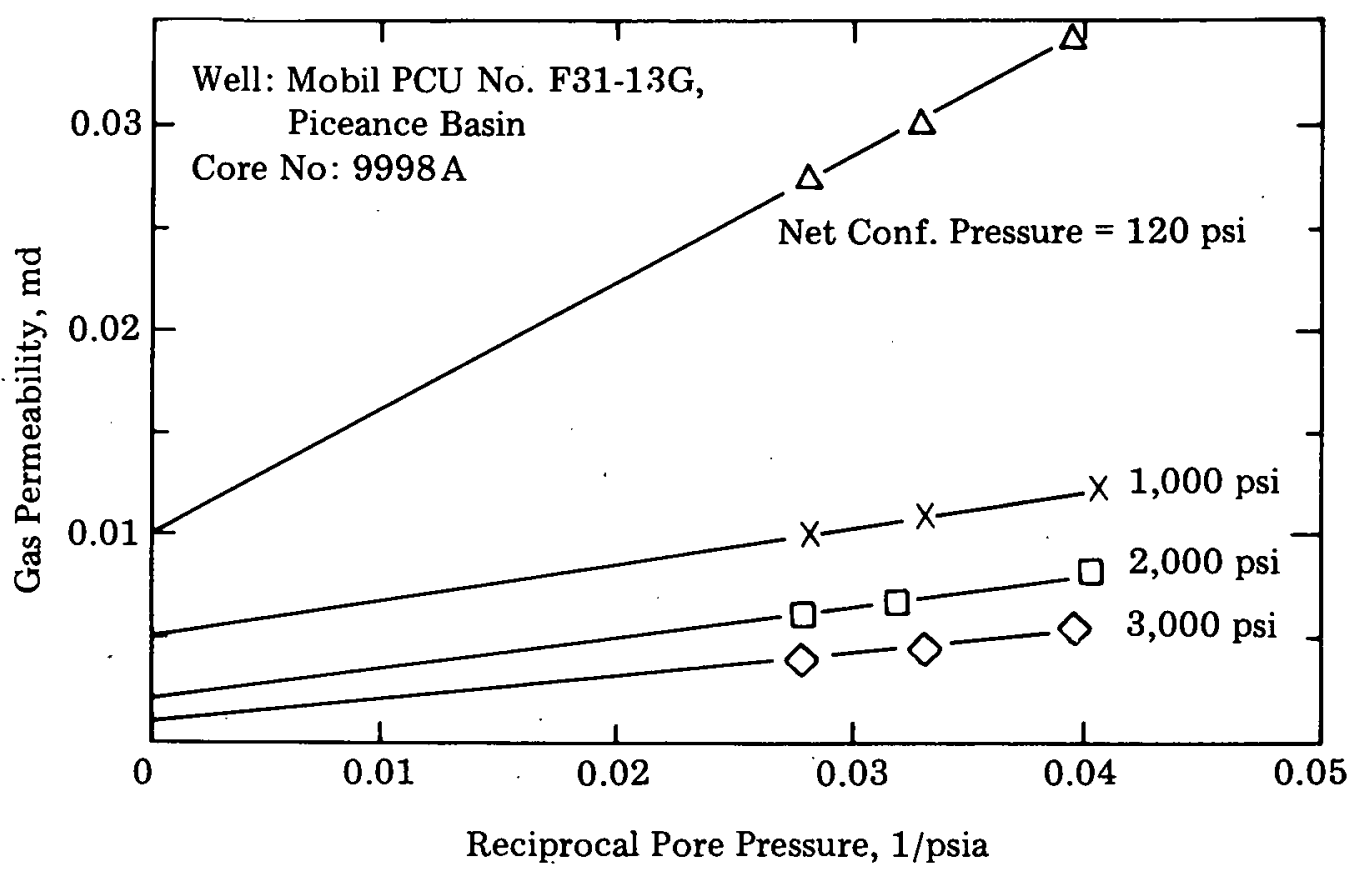

Figure 4-2 Effect of Net Confining Pressure on Gas Slippage for Flow Through a Partially Saturated Core

Table 4-3 Measurement of Net Confining Pressure on Gas Slippage Through a Partially Saturated Core

\begin{tabular}{cccr}
\hline \multicolumn{4}{c}{ Well: Mobil PCU No. F31-13G, Piceance Basin } \\
\multicolumn{4}{c}{ Core No: 9998A } \\
& Water Saturation: 20.9 percent & \\
\hline Net Confining & Slope & Intercept & b, \\
Pressure, psi & (md)(psi) & Ko, md & atm \\
\hline 120 & 0.608 & .01010 & 4.07 \\
1,000 & 0.174 & .00590 & 2.32 \\
2,000 & 0.160 & .00159 & 6.83 \\
3,000 & 0.119 & .00070 & 12.18 \\
& & & \\
\hline
\end{tabular}




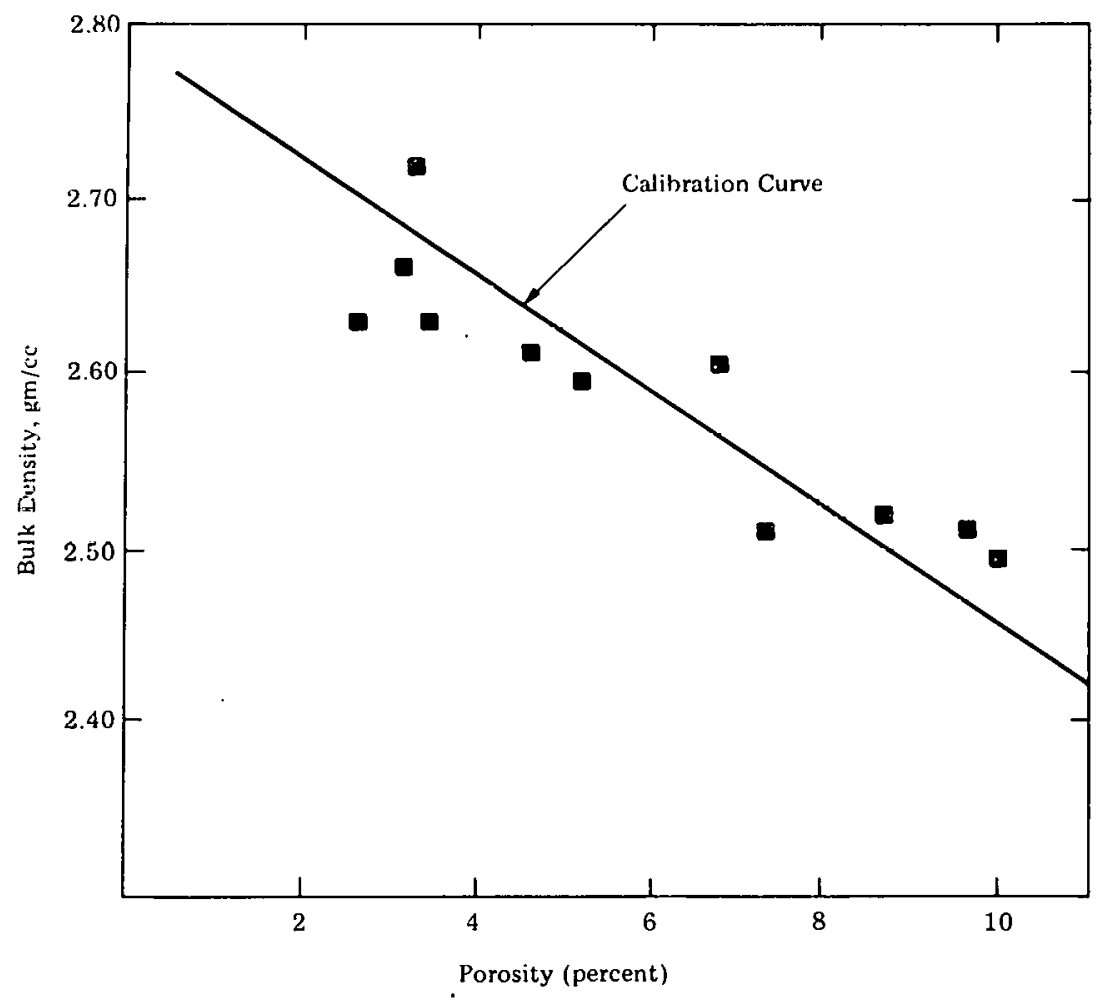

Figure 4-3 Density Log Calibration Curve for CIGE Natural Buttes Unit No. 21 Well

Eight sandstone cores have been acquired from the USGS Core Library to prepare samples which will cover a significant range of porosities and permeabilities for both laser and dielectric bridge measurements.

Study of Sonic, Neutron and Density Logging of Low Permeability Gas Sands - Texas A\&M University

Results of core, petrographic and sieve analyses indicate that a high density carbonate affects the porosity and matrix density of the examined core intervals from the CIGE

NBU No. 21 well. The low porosity zones (porosity less than 8 percent) have more carbonate cement and a higher matrix density than the high porosity zones (porosity greater than 8 percent), which have a matrix density comparable to sandstone $(2.65 \mathrm{gm} / \mathrm{cc})$. For this reason, the density log will underestimate the porosity of the highly cemented zoncs if a standard sandstone matrix density $(2.65$ $\mathrm{gm} / \mathrm{cc}$ ) is used for $\log$ calculations. Conversely, if a higher matrix density is used for log calculations (Schlumberger used 2.68 $\mathrm{gm} / \mathrm{cc}$ in a log run on the well), porosities will be overestimated in the zones with little carbonate cement. This exemplifies the inability of the density log to deal with variable matrix densities in a single formation. A calibration curve has been constructed to help solve this problem, as shown in Figure 4-3. Bulk density can be taken directly from the density log and converted to true porosity using this curve.

The feasibility of using supplemental or alternate logs to distinguish carbonates is under investigation. 
Because of the amount of time involved and the difficulty of saturating large cores, whole core analysis of the CIGE No. 21 has been discontinued. Table 4-4 is a comparison of whole core and plug analyses. The cores that have a high porosity give similar results from whole core or plug analysis. The low porosity cores give lower values for the whole core analysis than the plug analysis. This may be due to the difficulty in saturating the larger cores in the low porosity and low permeability zones.

Table 4-4 Summary of Whole Core vs. Plug Analysis for CIGE No. 21

\begin{tabular}{ccc}
\hline $\begin{array}{c}\text { Depth } \\
\text { (ft) }\end{array}$ & $\begin{array}{c}\text { Whole Core } \\
\text { Porosity } \\
\text { (percent) }\end{array}$ & $\begin{array}{c}\text { Plug } \\
\text { Porosity } \\
\text { (percent) }\end{array}$ \\
\hline $4,458.0$ & 1.70 & 4.26 \\
$4,458.5$ & 2.59 & 4.26 \\
$4,460.0$ & 1.72 & 4.26 \\
$4,463.0$ & 2.37 & 4.26 \\
$4,464.0$ & 1.50 & 4.26 \\
$4,457.5$ & 1.62 & 4.26 \\
$8,436.0$ & 8.10 & 8.66 \\
$8,436.5$ & 7.64 & 8.66 \\
$8,438.5$ & 3.87 & 8.66 \\
$4,442.0$ & 1.72 & $* *$ \\
$4,443.0$ & 1.70 & $* *$ \\
\hline
\end{tabular}

** No plugs measured from this interval

Use of Sonic Techniques to Measure In Situ Stresses - Lawrence Livermore Laboratory

Preliminary experiments were conducted in the laboratory to evaluate several different ways of using continuous wave and resonance techniques to measure acoustic velocity, which can be applied in the field. Shear wave velocity was measured in Nugget sandstone with two transducers mounted on a single face of the rock in a standard pulse transmission arrangement.

\section{Reservoir Simulation Studies}

Parametric Analysis of MHF Test Data and Engineering Studies of Western Gas Sands Intercomp

A report entitled "Multiple-Well Testing in Low Permeability Gas Sands" was completed. The report covers work done to investigate the feasibility of conducting a multiple-well test in the western gas sands.

The computational work necessary to prepare several sets of short flow time type curves has been completed. When drafting has been completed, these curves can be used to interpret pressure buildup data from fractured low permeability gas wells. Buildup data from this type of well usually followed a comparatively short flow period and the type curves that are now available are for the pressure response in a producing well. These type curves can be used for pressure buildup analysis only if the flow time preceding the buildup test is extremely long. The new type curves will not have this limitation.

\section{Schedule Status}

Figure 4-4 is a milestone chart depicting BETC progress within the WGSP.

\section{GAS RESEARCH INSTITUTE}

J. E. Evered (CER Project Manager) met with J. C. Sharer (GRI Project Manager) and S. D. Whitford (GRI Project Advisor) concerning development of a program plan, including generation of a matrix or information model to identify the state of the art in tight gas sands technology.

Evaluation of Seismic Data for Detailed Stratigraphic Studies of Lenticular Sands

Processing of data acquired from Site No. 1 (Bryson Canyon) is continuing. All data have 


\begin{tabular}{|c|c|c|c|c|c|c|c|c|c|c|c|c|c|}
\hline \multirow{2}{*}{\multicolumn{2}{|c|}{\begin{tabular}{|c|} 
WGSP - BETC \\
RESOURCE ASSESSMENT
\end{tabular}}} & \multicolumn{12}{|c|}{ FY -80} \\
\hline & & OCT & Nov & DEC & JAN & FEB & MAR & APR & MAY & JUN & JUL & AUG & SEP \\
\hline \multicolumn{14}{|c|}{ FORMATION EVALUATION } \\
\hline \multicolumn{14}{|c|}{$\begin{array}{l}\text { IMPROVED PRESSURE CORING } \\
\text { SYSTEM }\end{array}$} \\
\hline \multicolumn{14}{|c|}{ Core Retriever Design \& Test } \\
\hline \multicolumn{14}{|c|}{ Core Fluid Testing } \\
\hline \multirow{2}{*}{\multicolumn{14}{|c|}{ Bit Design \& Fabrication }} \\
\hline \multirow{2}{*}{\multicolumn{14}{|c|}{ Bit Tests }} \\
\hline & & & & & & & & & & & & & \\
\hline \multicolumn{14}{|c|}{ IN SITU PERMEABILITY } \\
\hline \multirow{2}{*}{\multicolumn{14}{|c|}{$\begin{array}{l}\text { Measurement of Formation } \\
\text { Characteristics }\end{array}$}} \\
\hline & & & & & & & & & & & & & \\
\hline \multicolumn{14}{|l|}{ LEGEND } \\
\hline$\nabla$ & $\begin{array}{l}\text { Scheduled Start and Completion } \\
\text { of Task }\end{array}$ & & & & & & & & & & & & \\
\hline$\nabla$ & Completed Milestone & & & & & & & & & & & & \\
\hline$\overline{0}$ & Task Progressing & & & & & & & & & & & & \\
\hline 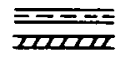 & $\begin{array}{l}\text { Task Progress Not Reported } \\
\text { Delay in Work on Task }\end{array}$ & & & & & & & & & & & & \\
\hline
\end{tabular}

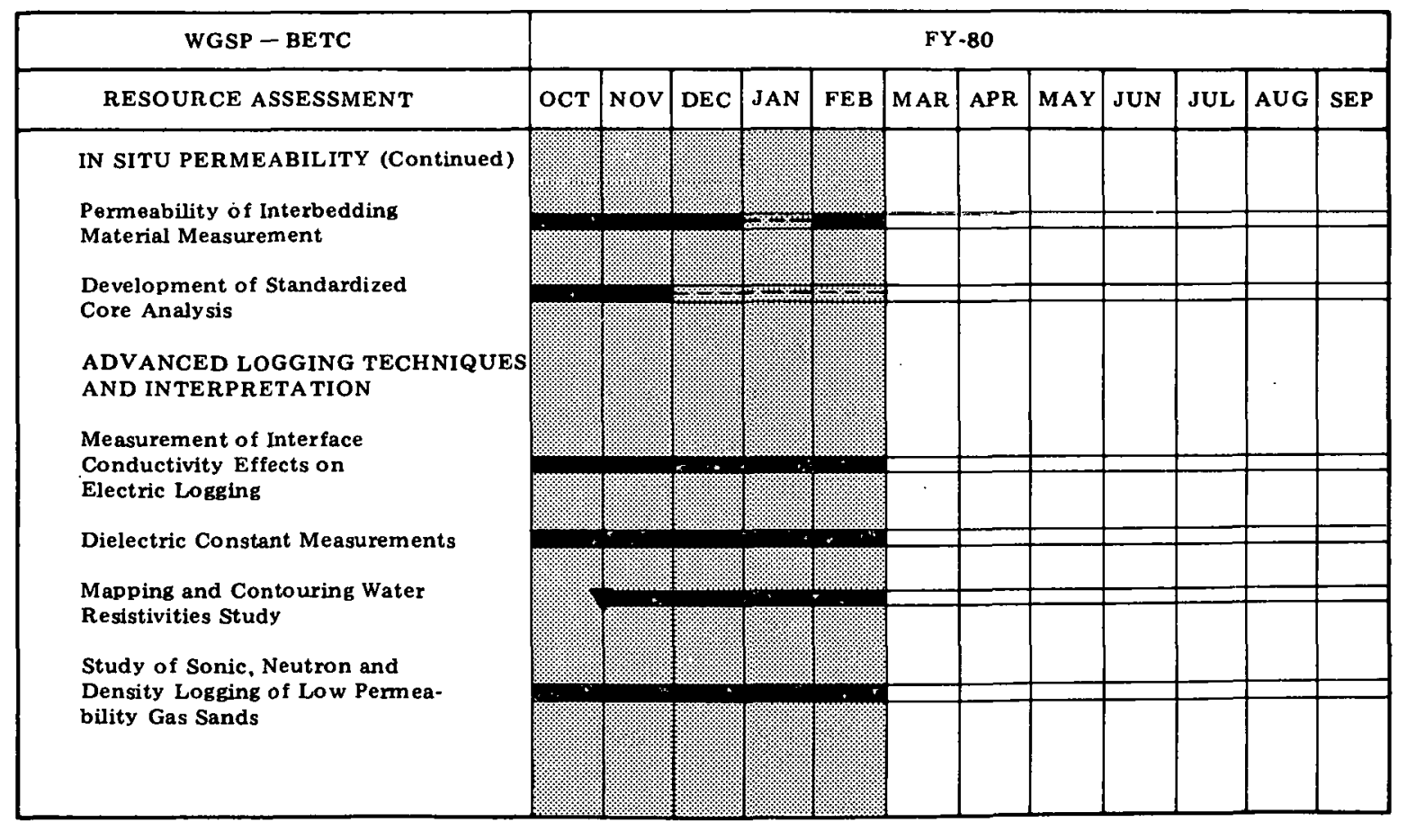

Figure 4-4 Milestone Chart-BETC 


\begin{tabular}{|c|c|c|c|c|c|c|c|c|c|c|c|c|}
\hline WGSP - BETC & \multicolumn{12}{|c|}{ FY -80} \\
\hline RESOURCE ASSESSMENT & OCT & Nov & DEC & JAN & FEB & MAR & APR & MAY & JUN & JUL & AUG & SEP \\
\hline \multicolumn{13}{|l|}{$\begin{array}{l}\text { STIMULATION MECHANICS \& } \\
\text { METHODS }\end{array}$} \\
\hline $\begin{array}{l}\text { Perform Analysis of MHF Test } \\
\text { Data and Engineering Studies } \\
\text { ROCK-FLUID INTERACTION }\end{array}$ & & & & & & & & & & & & \\
\hline \multicolumn{13}{|l|}{$\begin{array}{l}\text { PROPPANT AND PROPPANT } \\
\text { TRANSPORT }\end{array}$} \\
\hline \multicolumn{13}{|l|}{$\begin{array}{l}\text { Evaluation of Particle Transport } \\
\text { in Proppant Beds }\end{array}$} \\
\hline \multicolumn{11}{|l|}{$\begin{array}{l}\text { Evaluation of Proppant Embed- } \\
\text { ment in Tight Gas Sands }\end{array}$} & & \\
\hline \multicolumn{13}{|l|}{ Study of Proppant Settling } \\
\hline $\begin{array}{l}\text { Light Weight Proppant Feusi- } \\
\text { bility Study }\end{array}$ & & & & & & & & & & & & \\
\hline
\end{tabular}

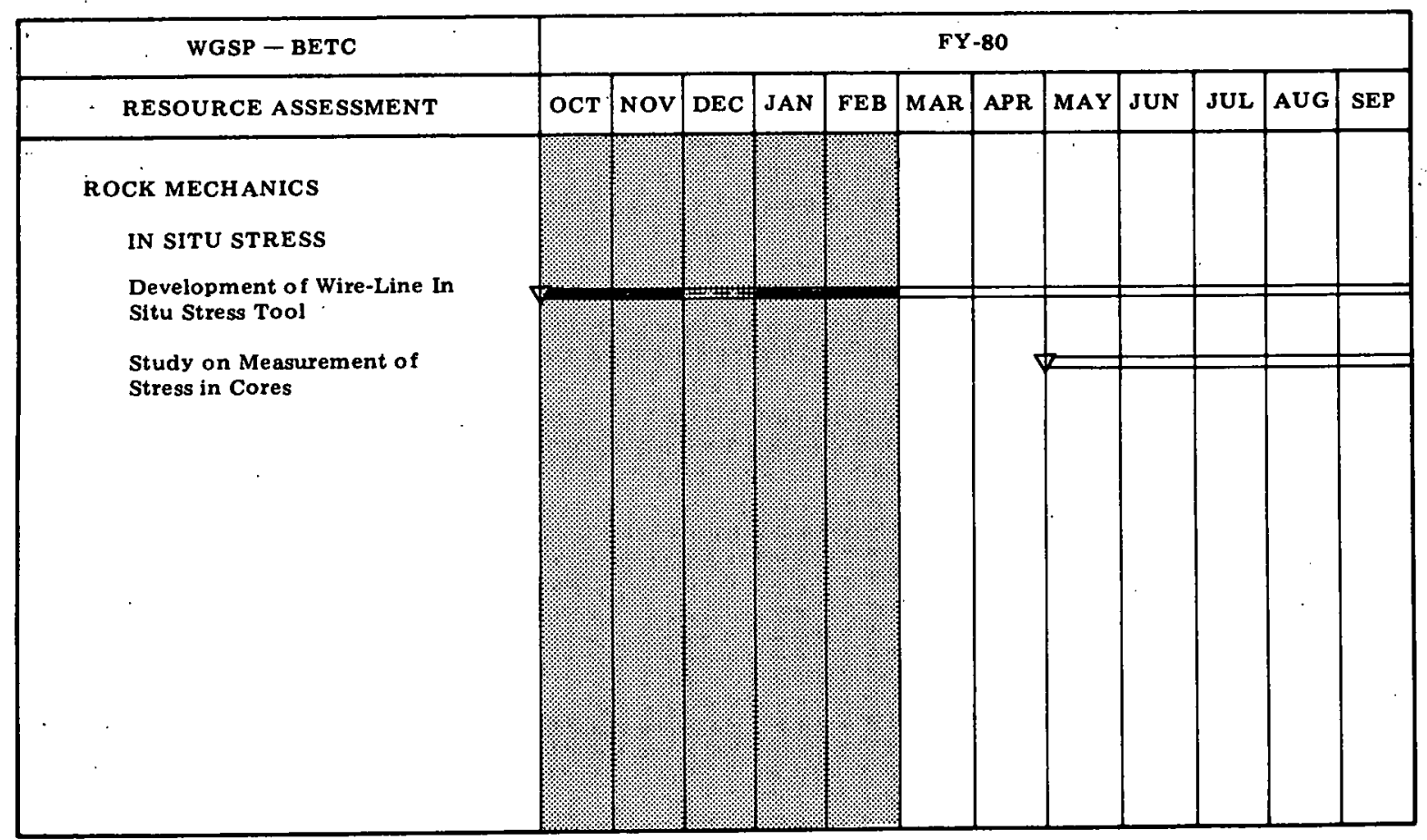

Figure 4-4 Continued 
been compiled into two dimensional (X-T) record sections, which have been analyzed for frequency content and continuity of events across the site. Full three-dimensional analysis of these data will be performed, followed by interpretation of the seismic data regarding detection and delineation of sand channels.

\section{LAWRENCE LIVERMORE LABORATORY}

\section{Theoretical Analysis}

Two sets of calculations were made with the time dependent finite element model to quantify the changes in material response due to a variation in density. In the problem geometry of Figure 4-5, a crack initiates at $x=0, y=0$, propagates bilaterally at half the dilatational wave speed for a distance "c", and stops. The result is the time history of the $y$ displacement of a point just behind the final position of the right crack tip. In the calculations, the elastic constants did not vary across the interface but in some cases the densities did. The Lame constants were set at $30 \mathrm{GPa}$ each and the density of material 1 (Figure 4-5) was always $2.7 \mathrm{gm} / \mathrm{cm}^{3}$. However, the density of the second material was varied from 0.3 to $24.3 \mathrm{gm} / \mathrm{cm}^{3}$. Obviously the largest value of density does not correspond to any known material, but a large variation was made to determine the magnitude of the effect of density variation on material overshoot.

The results are given for two final crack lengths. In Figure 4-6, the perpendicular nondimensional displacement is plotted as a function of nondimensional time (crack initiates at $t_{d}=0$ and crack stops at $t_{d}=1$ ) when $c / L=0.6$. Thus the crack stops approximately as scaled in Figure 4-5. The rather large change in density did not affect the material response to a large degree.

A similar plot is shown in Figure 4-7, but here the crack propagates to the interface and stops $(c / L=1.0)$. The variation in material response is larger for this case than for the previous one and the line corresponding to the least dense material crosses the other two lines at later times. There is a change in material response, but even in the last case, this change is not large when viewed as a fraction of the maximum displacement.

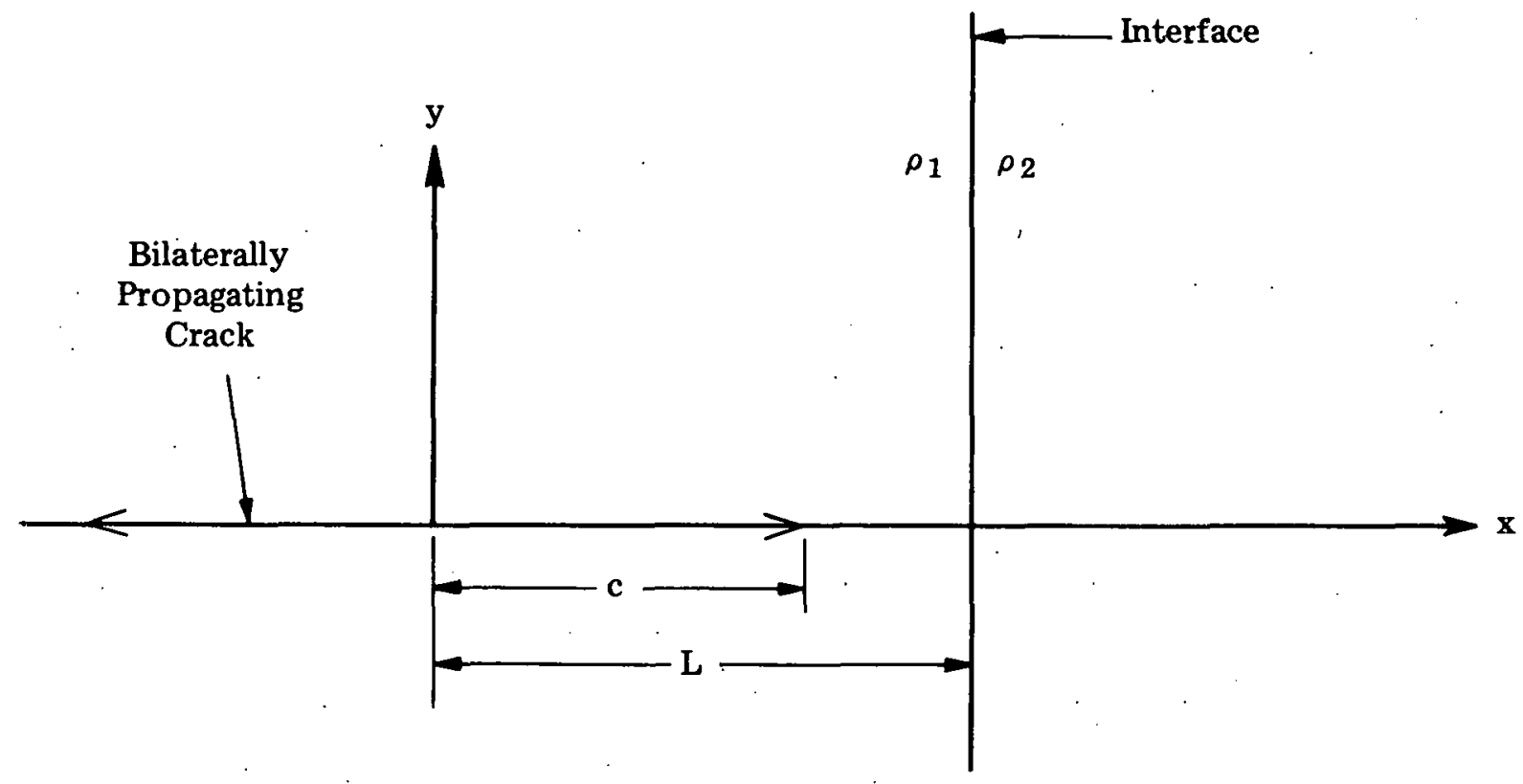

Figure 4-5 Problem Geometry for Time Dependent Crack Propagation Near An Interface 


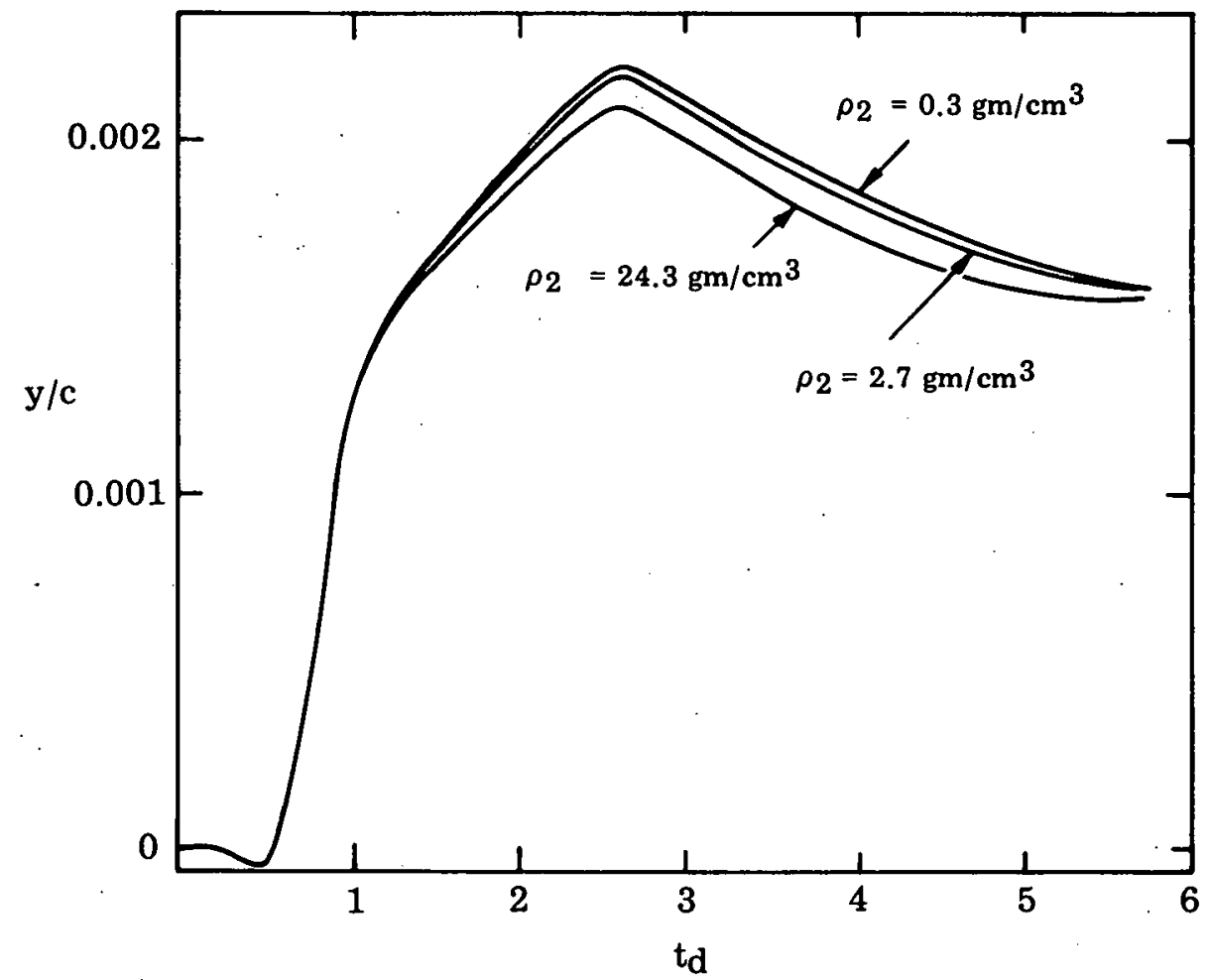

Figure 4-6 Displacement Perpendicular to the Crack when $c / \dot{L}=0.6$ (the crack does not reach the interface).

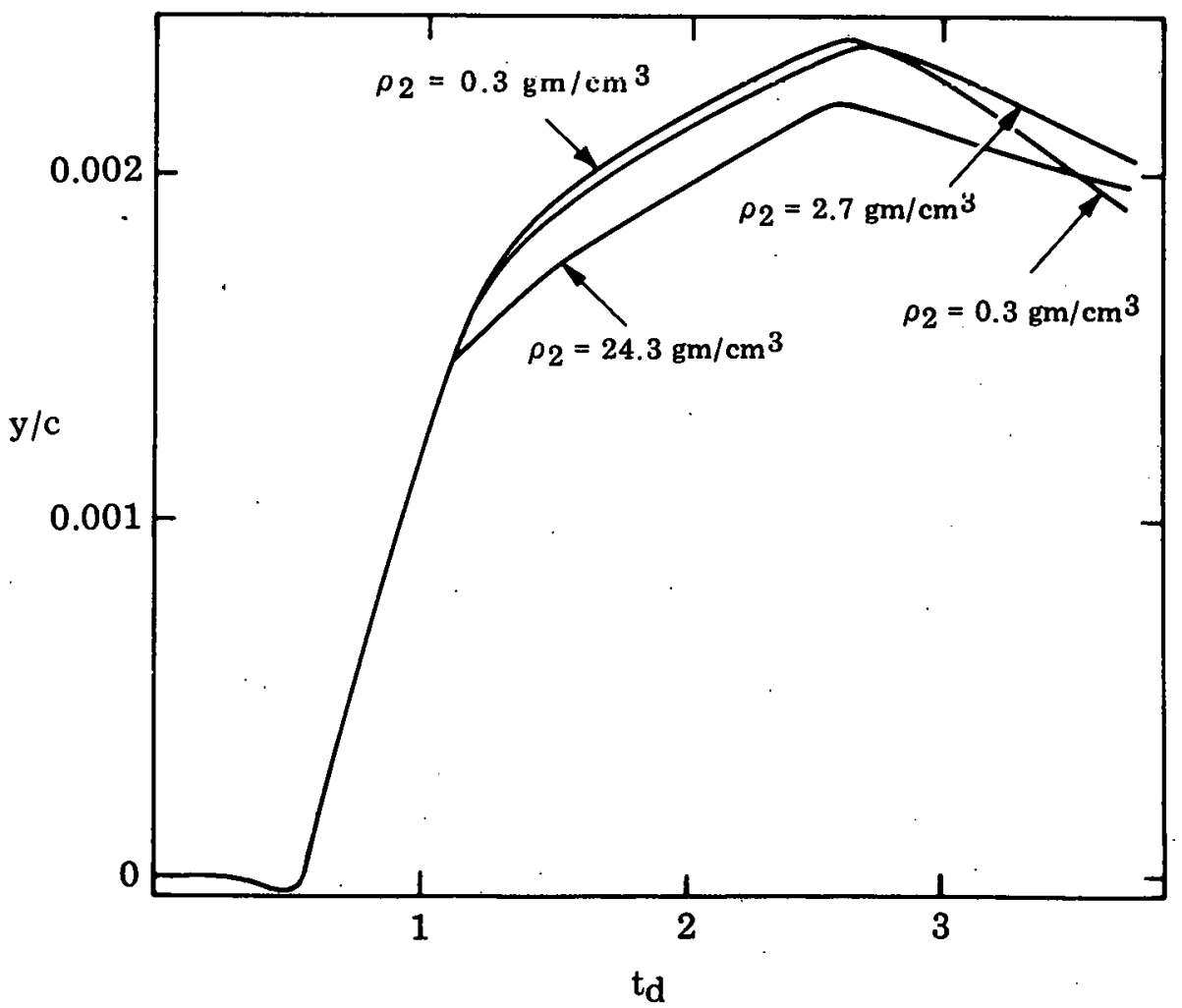

Figure 4-7 Displacement Perpendicular to the Crack when $c / L=1.0$ (the crack stops at the interface). 


\section{Laboratory Experiments}

Frictional Effects on Crack Growth Across Interfaces

During February, experiments were performed to study hydraulic crack penetration of lubricated unbonded interfaces in Nugget sandstone. These experiments were similar to the experiments on Indiana limestone reported in January's Status Report. The C-100 lubricant was used. The threshold normal stress for cracks to cross lubricated unbonded interfaces in the sandstone was about 1,700 psi, compared to about 800 psi for the unlubricated interfaces. At a normal load of $1,700 \mathrm{psi}$, friction experiments indicate that the lubricated interfaces can support a shear stress of about 300 psi before slippage occurs. At a normal load of 800 psi, friction experiments indicate that the smooth unlubricated interfaces can support a frictional shear stress of about 400 psi before slippage occurs.

The experiments on the limestone and sandstone show that as the interfaces are lubricated, which increases the threshold normal stress for crack penetration, the threshold frictional shear stress is decreased.

\section{Rock Mechanics Measurements}

Pressure-volume measurements continued on samples of Mesaverde sandstone from Rio Blanco County, Colorado; and are nearly complete. Testing has begun on the high pressure system for simultaneous ultrasonic velocity measurements in multiple directions under confining pressure. Preparation has begun on test specimens of Mesaverde shale and sandstone from Colorado and Wyoming for these ultrasonic measurements.

\section{Geology and Geophysics}

Work during February consisted of a review and evaluation of Piceance Basin data on the structure, natural fractures, and mini hydraulic fractures used in attempted measurement of in situ stress. This was in the Green River Formation with the following preliminary results.

- Minor folds seem due to differential vertical movements rather than compression. Minor extensional normal faults are oriented parallel to the fold axis, giving an apparent lesser horizontal stress perpendicular to, and a greater stress parallel to, the fold axis.

- The major sets of natural surface fractures strike parallel to the faults and presumed greater horizontal stress.

The manuscript, "A review of the mechanics and occurrence of natural fractures in rock as applied to the development of the tight western gas sands," was revised and delivered for processing as an LLL report.

\section{Schedule Status}

The progress of LLL within the WGSP is shown in Figure 4-8, a milestone chart.

\section{LOS ALAMOS SCIENTIFIC LABORATORY}

\section{Nuclear Magnetic Resonance Studies}

Work in this area focused on two different tasks. The $20 \mathrm{MHz}$ pulsed NMR spectrometer was activated. Upon activation, preliminary sensitivity measurements were made on glycerol and water saturated sands. Also, a computer literature search was initiated on the application of NMR to geosciences.

\section{Schedule Status}

Figure $4-9$ is a milestone chart depicting LASL's WGSP progress. 


\begin{tabular}{|c|c|c|c|c|c|c|c|c|c|c|c|c|c|}
\hline \multicolumn{2}{|r|}{ WGSP - LLL } & \multicolumn{12}{|c|}{ FY -80} \\
\hline & & OCT & NOV & DEC & JAN & FEB & MAR & APR & MAY & JUN & JUL & AUG & SEP \\
\hline \multicolumn{14}{|c|}{$\begin{array}{l}\text { MODEL DEVELOPMENT AND } \\
\text { APPLICATION }\end{array}$} \\
\hline \multicolumn{14}{|c|}{2 Dimension Application } \\
\hline \multicolumn{14}{|c|}{$\begin{array}{l}\text { LABOR ATORY EXPERIMENTAL } \\
\text { MODELING }\end{array}$} \\
\hline \multicolumn{14}{|c|}{ Interface and Friction Experiments } \\
\hline \multicolumn{14}{|c|}{ Pore Pressure Experiments } \\
\hline LEGEND & & & & & & & & & & & & & 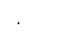 \\
\hline 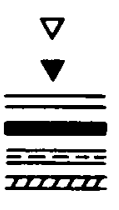 & $\begin{array}{l}\text { Scheduled Start and Completion } \\
\text { of Task } \\
\text { Completed Milestone } \\
\text { Projected Schedule } \\
\text { Task Progressing } \\
\text { Task Progress Not Reported } \\
\text { Delay in Work on Task }\end{array}$ & & & & & & & & & & & & \\
\hline
\end{tabular}

\begin{tabular}{|c|c|c|c|c|c|c|c|c|c|c|c|c|}
\hline \multirow[t]{2}{*}{ WGSP - LLL } & \multicolumn{12}{|c|}{$F Y-80$} \\
\hline & OCT & Nov & DEC & JAN & FEB & MAR & APR & MAY & JUN & JUL & AUUG & SEP \\
\hline \multicolumn{13}{|l|}{ MECHANICAL PROPERTIES } \\
\hline \multicolumn{13}{|l|}{ Tight Sand Measurements } \\
\hline \multicolumn{13}{|l|}{$\begin{array}{l}\text { GEOLOGIC AND GEOPHYSICAL } \\
\text { DATA }\end{array}$} \\
\hline \multicolumn{13}{|l|}{ Evaluation of Core and Log Data } \\
\hline \multicolumn{13}{|l|}{ Reservolr Characterization } \\
\hline \multirow{3}{*}{\multicolumn{13}{|c|}{$\begin{array}{l}\text { MEASUREMENT OF IN SITU } \\
\text { PERMEABILITY OF INTERBEDDING } \\
\text { MATERIAL OF TIGHT GAS SANDS }\end{array}$}} \\
\hline & & & & & & & & & & & & \\
\hline & & & & & & & & & & & & \\
\hline \multirow{2}{*}{\multicolumn{13}{|c|}{$\begin{array}{l}\text { Measurement System } \\
\text { Design and Construction }\end{array}$}} \\
\hline & & & & & & & & & & & & \\
\hline \multicolumn{13}{|l|}{ Preliminary Testing } \\
\hline \multicolumn{13}{|l|}{ Test Control Data Acquisition } \\
\hline Final Report & & & & & & & & & & & & \\
\hline
\end{tabular}

Figure 4-8 Milestone Chart - LLL 


\begin{tabular}{|c|c|c|c|c|c|c|c|c|c|c|c|c|c|}
\hline \multirow{2}{*}{\multicolumn{2}{|c|}{$\begin{array}{l}\text { WGSP - LASL } \\
\text { REMOTE CHARACTERIZATION OF } \\
\text { FLUID SATURATIONS }\end{array}$}} & \multicolumn{12}{|c|}{ FY-80 } \\
\hline & & OCT & Nov & DEC & JAN & $F^{\prime} E B$ & MAR & APR & MAY & JUN & JUL & AUG & SEP \\
\hline \multirow{3}{*}{\multicolumn{2}{|c|}{$\begin{array}{l}\text { PERMEABILITY AND POROSITY } \\
\text { DETERMINATIONS ON CORE } \\
\text { SAMPLES } \\
\text { GEOLOGICAL STUDIES: Corre- } \\
\text { lation Studies between LOg Suites, } \\
\text { Corc Scctions, and Lab Meusure- } \\
\text { ments. }\end{array}$}} & & & & & & & & & & & & \\
\hline & & & & & & & & . & & & & & \\
\hline & & & & & & & & & & & & & \\
\hline \multicolumn{14}{|l|}{ LEGEND } \\
\hline 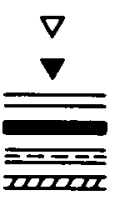 & $\begin{array}{l}\text { Scheduled Start and Completion } \\
\text { of Task } \\
\text { Completed Milestone } \\
\text { Projected Schedule } \\
\text { Task Progressing } \\
\text { Task Progress Not Reported } \\
\text { Delay in Work on Task }\end{array}$ & & & & & & & & & & & & \\
\hline
\end{tabular}

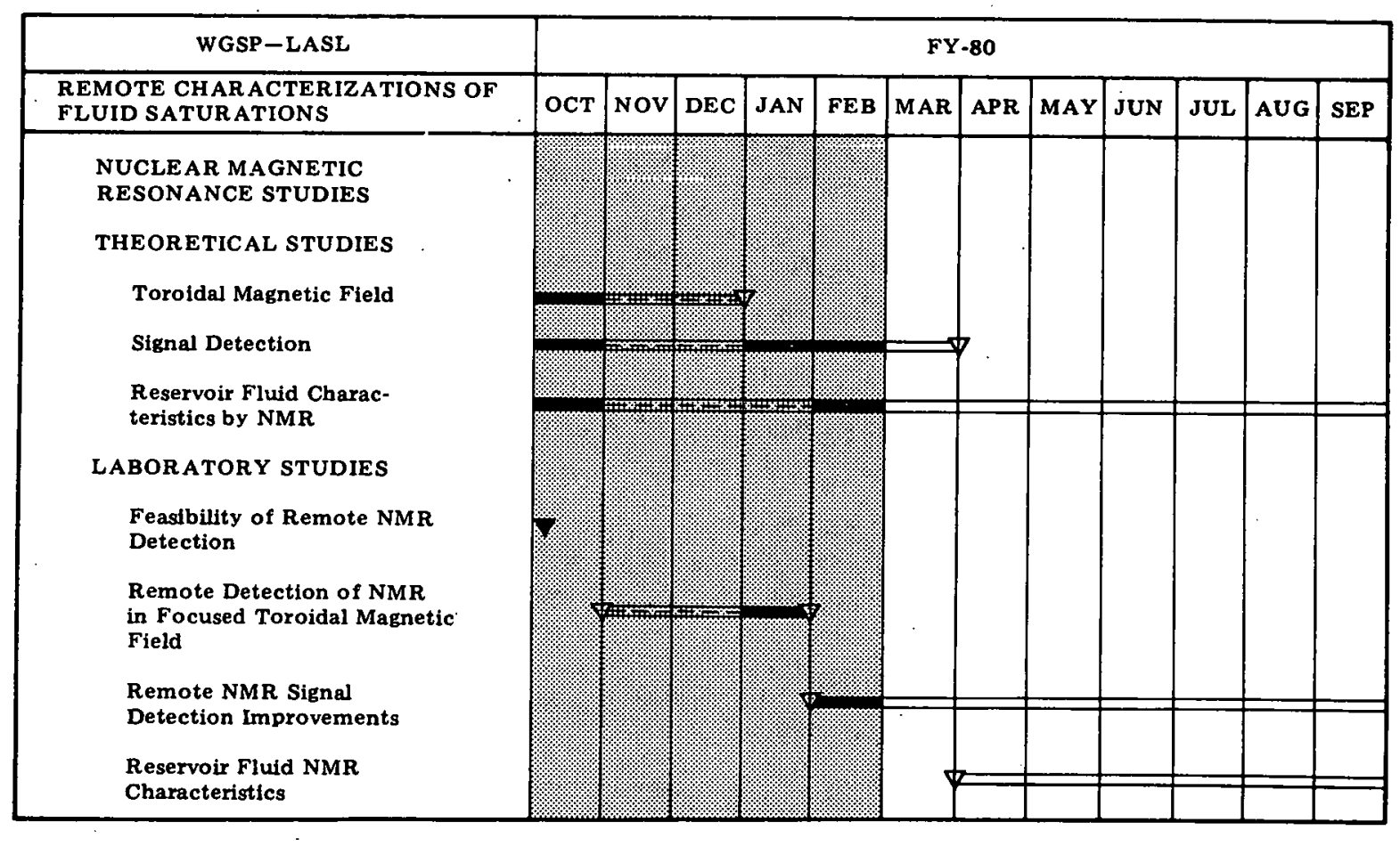

Figure 4-9 Milestone Chart-LASL 


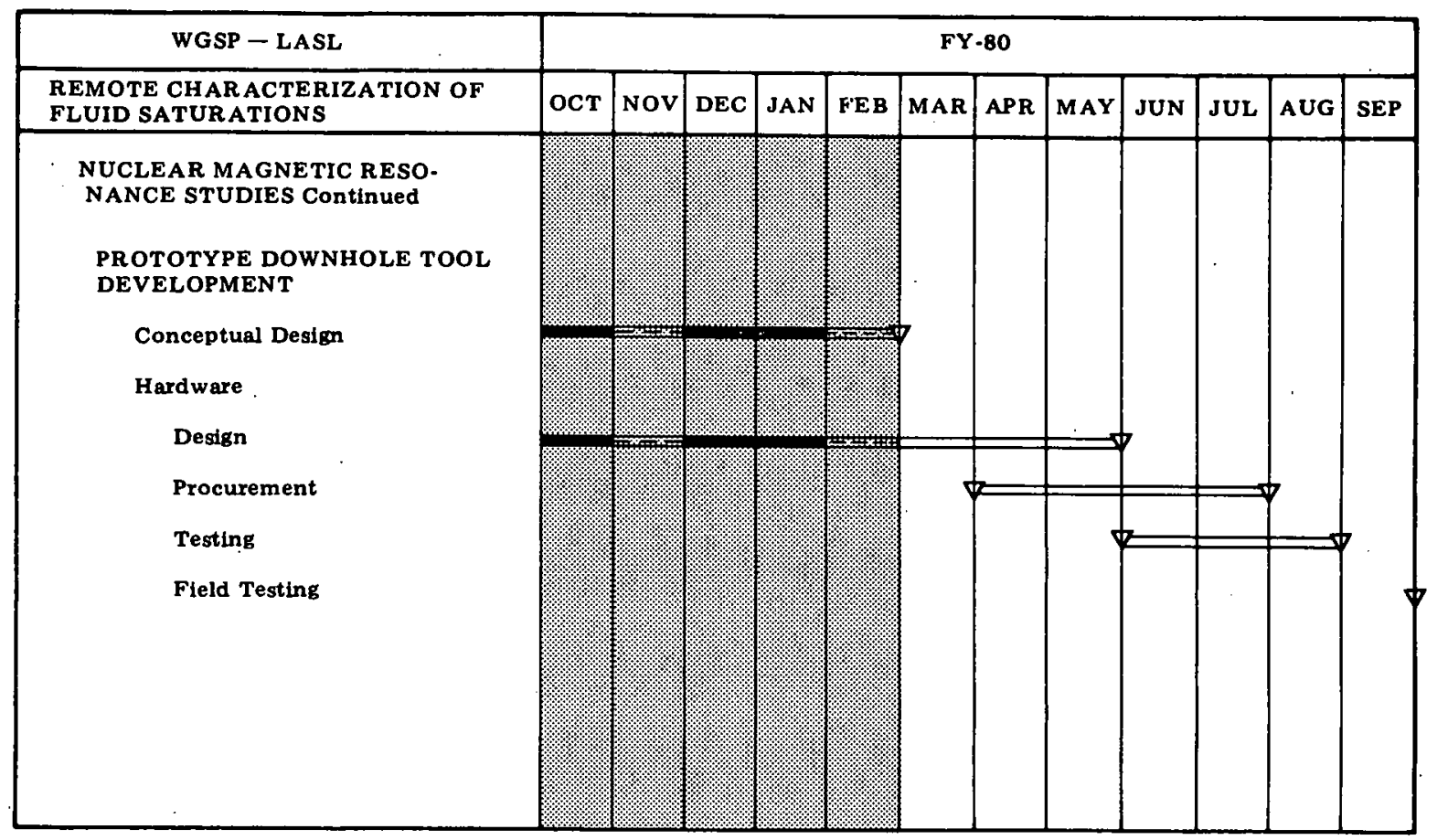

Figure 4-9 Continued

\section{SANDIA LABORATORIES}

\section{Hydraulic Fracture Characterization}

Sandia participated in a multifaceted experiment with Shell Oil Company at their Big Mineral Creek field near Sherman, Texas on February 26 and 27. Three wells were involved in this combined seismic and surface electrical potential fracture mapping experiment. The main fracture was produced with $34,000 \mathrm{gal}$ of fluid at $5,800 \mathrm{ft}$ in the " $\mathrm{S}$ " sands, with an estimated radius of $360 \mathrm{ft}$.

The surface electrical potential system (SEPS) was fielded at radii of 1,200 and $1,600 \mathrm{ft}$ (48 test probes) around the fractured well No. 343. Current was injected into the formation at $5,700 \mathrm{ft}$ by the current injection probe. Two current sinks were used: the first was another well located approximately $8,900 \mathrm{ft}$ to the south-southeast; and the second sink was that of the fractured well's casing. Although both methods produced signals of comparable strength, the fractured well's casing produced waveshapes with almost no spectral distortion. The potential measurement boxes (PMBs) which monitor the probe potentials, were increased to a gain of $72 \mathrm{db}$ from a previous gain of approximately $42 \mathrm{db}$. Apparently this was possible because of the increased risetime of the injected current pulse and the use of shielded current wire throughout the PMB portion of the system.

Although good signals were obtained, a fracture length of $360 \mathrm{ft}$ minimized the possibility of seeing this from the surface. However, the success seems to be more from the improvement of the surface current injection system. 


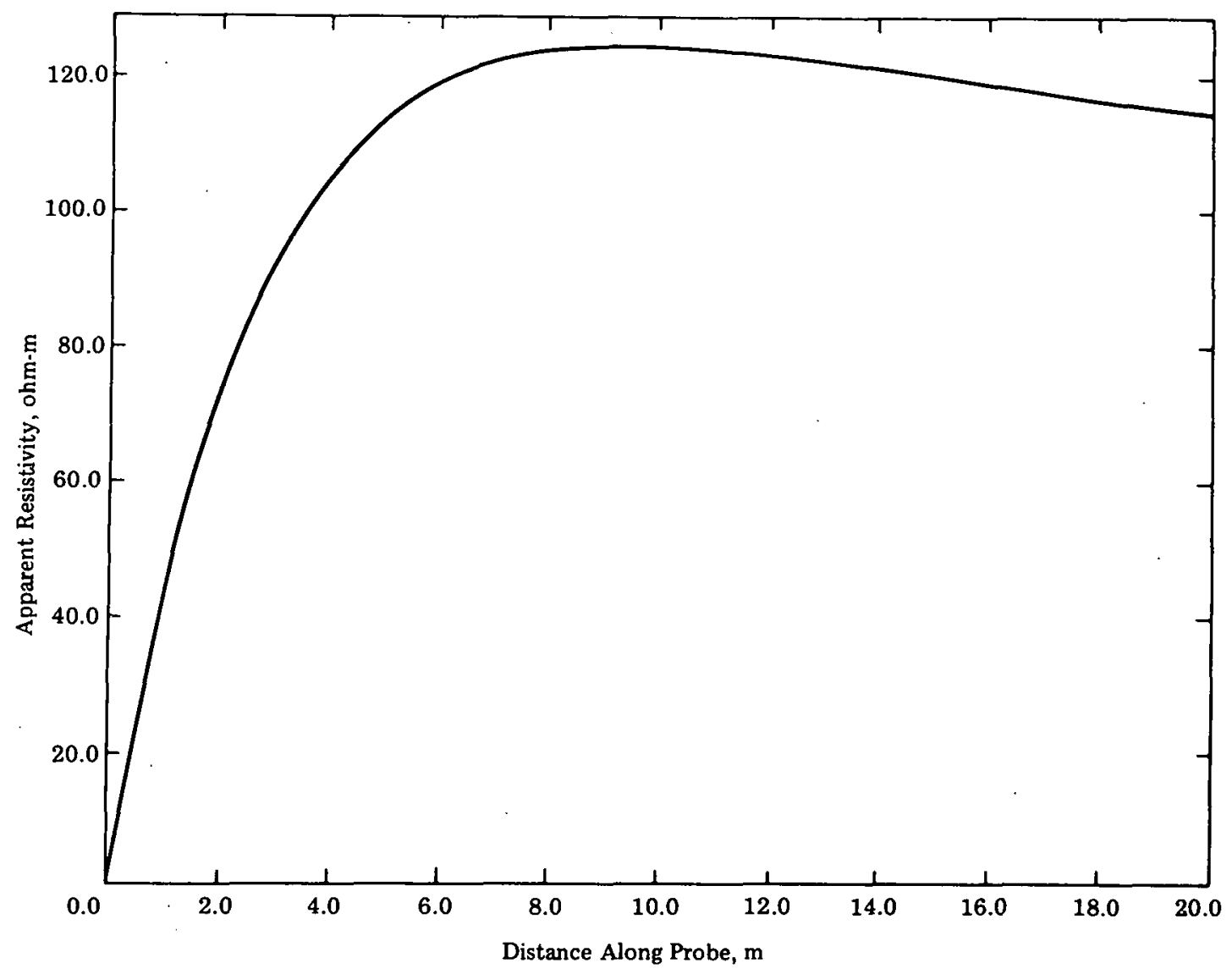

Figure 4-10 Resistivity as a Function of Position Along the Probe for an Exponential Resisitivity Distribution

On February 26, a breakdown test was performed in Unit No. 343. Borehole seismic units were placed in that well just below the fracture interval and in well No. 309 at approximately the same depth. Well No. 309 is about $560 \mathrm{ft}$ east of No. 343. Fracture signals were recorded for a breakdown of approximately $75 \mathrm{BBL}$ in each stage. The borehole seismic unit in No. 343 was moved to well No. 210 for the main fracture of 34,000 gal on February 27. Seismic fracture signals were recorded in both wells, No. 309 and No. 210, during the main fracture.

\section{Borehole Resistivity System}

The numerical solution to the forward onedimensional resistivity problem was programmed and tested on various resistivity profiles. The apparent resistivity as a function of position along the probe for an exponential resistivity distribution is given in Figure 4-10. The probe was assumed to be $3 \mathrm{in}$. in diameter with a 3 -in. long current electrode at its center. The resistivity in this example varied from 1 ohm-m at the probe to 100 ohm-m far from the probe with a relaxation distance of $1 / 4 \mathrm{~m}$. The overshoot in the apparent resistivity to a value near 125 ohm-m is $9 \mathrm{~m}$ from the current electrode.

This forward solution will be used:

- to test R. Langer's inverse method, - to compare with a layered approximation, and

- possibly as the forward solution in a ridge regression inversion. 
Table 4-5 Final Core Analysis Results for Eleven Samples from CIGE No. 21

\begin{tabular}{|c|c|c|c|c|c|c|c|c|c|}
\hline \multirow[b]{2}{*}{$\begin{array}{c}\text { Sample Depth } \\
\text { (ft) }\end{array}$} & \multirow[b]{2}{*}{$\begin{array}{c}\text { Helium } \\
\text { Porosity } \\
\text { (pexcent) }\end{array}$} & \multirow[b]{2}{*}{$\begin{array}{l}\text { "A" Brine } \\
\text { Porosity } \\
\text { (percent) }\end{array}$} & \multirow[b]{2}{*}{ FFI } & \multirow[b]{2}{*}{$\begin{array}{c}\text { Air } \\
\text { Permeability } \\
\text { (md) }\end{array}$} & \multirow[b]{2}{*}{$\begin{array}{l}\text { "A" Brine } \\
\text { Permeabillity } \\
\text { (md) }\end{array}$} & \multirow{2}{*}{$\begin{array}{c}\text { Apparent } \\
\text { Sand Grain } \\
\text { Density } \\
\left(\mathbf{g} / \mathrm{cm}^{3}\right)\end{array}$} & \multicolumn{3}{|c|}{$\mathbf{T}_{1}$ Relaxation (ms) } \\
\hline & & & & & & & $\mathbf{A} / \mathbf{T}_{1} \mathbf{A}$ & $\mathbf{B} / \mathbf{T}_{\mathbf{1 B}}$ & $\mathbf{C} / \mathbf{T}_{1} \mathbf{C}$ \\
\hline $4,403.3$ & 8.8 & 8.7 & 9.7 & 0.05 & 0.02100 & 2.72 & $\frac{.440}{16.5}$ & $\frac{.560}{0.33}$ & \\
\hline $4,403.8$ & 9.2 & 9.2 & 9.3 & 0.90 & 0.35000 & 2.66 & $\frac{.518}{279.7}$ & $\frac{.309}{24.2}$ & $\frac{.173}{2.3}$ \\
\hline $4,478.3$ & 7.0 & 7.0 & 8.7 & 0.20 & 0.13000 & 2.69 & $\frac{.372}{281.2}$ & $\frac{.338}{19.8}$ & $\frac{.291}{1.3}$ \\
\hline $4,478.3$ & 4.7 & 4.5 & 5.6 & $<0.05$ & 0.00470 & 2.68 & $\frac{.297}{92.8}$ & $\frac{.302}{7.9}$ & $\frac{.401}{1.0}$ \\
\hline 6.492 .0 & 9.6 & 9.6 & 10.5 & 0.30 & 0.20000 & 2.65 & $\frac{.313}{195.1}$ & $\frac{.514}{31.0}$ & $\frac{.172}{3.7}$ \\
\hline $6,493.0$ & 9.8 & 9.6 & 10.6 & 0.20 & 0.06100 & 2.65 & $\frac{.253}{228.4}$ & $\frac{.516}{41.0}$ & $\frac{.231}{5.3}$ \\
\hline $7,469.0$ & 3.7 & 3.7 & 4.4 & $<0.05$ & 0.00045 & 2.70 & $\frac{.177}{55.7}$ & $\frac{.485}{8.7}$ & $\frac{.338}{1.7}$ \\
\hline $7,469.0$ & 4.7 & 4.7 & 5.6 & $<0.05$ & 0.00090 & 2.70 & $\frac{.329}{37.5}$ & $\frac{.566}{6.3}$ & $\frac{.106}{1.1}$ \\
\hline 8.492 .0 & 9.4 & 9.1 & 9.8 & 0.10 & 0.02700 & 2.67 & $\frac{.344}{278.3}$ & $\frac{.438}{49.5}$ & $\frac{.218}{8.5}$ \\
\hline $8,496.5$ & 2.7 & 2.6 & 4.0 & 0.20 & $0.77000 *$ & 2.47 & $\frac{.262}{98.5}$ & $\frac{.280}{8.7}$ & $\frac{.458}{1.1}$ \\
\hline $8,525.0$ & 6.1 & 6.0 & 6.8 & $<0.05$ & 0.01600 & 2.67 & $\frac{.329}{175.0}$ & $\frac{.464}{27.2}$ & $\frac{.208}{5.3}$ \\
\hline
\end{tabular}

* Laminated Sample

High Resolution Seismic Formation Mapping

Processing of the Site No. 1 (Bryson Canyon) data continued. All data have been compiled and segregated into two-dimensional (X-T) record sections.

\section{Nuclear Magnetic Logging}

The proton nuclear magnetic resonance was studied for 11 core samples of low permeability gas sands from the CIGE No. 21 well, Uinta Basin. The cores were prepared by machining the samples to size, followed with toluene cleaning, vacuum drying, and brine saturation. A high salinity, deoxygenated brine was used to minimize swelling of the clays. To insure saturation, the samples were pressurized in the brine solution for several hours and stored in the brine prior to obtaining the measurements. For calibration of the NMR, a standard sample was also prepared, which consisted of a 10 percent mixture of $\mathrm{H}_{2} \mathrm{O}$ in $\mathrm{D}_{2} \mathrm{O}$, magnetically doped to have about the same relaxation time $(\mathrm{T})$, as the rock samples.

For each specimen, the free induction decay produced by a $90^{\circ}$ pulse at $10 \mathrm{MHz}$ resonance was sampled at $50,100,200$, and 500 microseconds. The magnetization was found to decay exponentially over this interval. This signal, when extrapolated to $\mathrm{T}=0$ and compared with the standard 10 percent sample, yielded a free fluid index (FFI) for the specimen. The FFI obtained for each sample is listed in Table 4-5. They are in fairly good agreement with the producible porosities, $\phi_{\mathrm{p}}$, deduced by standard core analysis on the same samples. 
The spin-lattice relaxation was measured for each specimen by sampling the magnetization 50 microseconds after a $180^{\circ}-t$ $90^{\circ}$ pulse sequence, where the pulse separation, $t$, was varied from 0.6 to 600 milliseconds. This relaxation was found to be nonexponential; thus, no single $\mathrm{T}_{1}$ could be ascribed. Rather, the data were computer fit to a three-exponential decay

$$
\begin{aligned}
& M(t)=M(o) A \exp \left(-t / T_{1 A}\right)+ \\
& B \exp \left(-t / T_{1 B}\right)+C \exp \left(-t / T_{1} C\right)
\end{aligned}
$$

A non-linear least-squares procedure was used to obtain the six adjustable amplitude and decay parameters. This procedure was found to produce a good fit to the observed relaxation for each sample, except for sample No. 1, where a two-component decay was found sufficient. The decay parameters for the samples are listed in Table 4-5. These parameters describe the fraction of the protons residing in the larger pores (the larger $T_{1} s$ ) relative to those in the smaller pores (the smaller $\mathrm{T}_{1} \mathrm{~s}$ ). They will be used in various models to generate permeabilities. These will be compared with the brine permeabilities $\mathrm{K}_{\mathrm{b}}$, listed in Table 4-5.

\section{Electromagnetic Logging}

\section{Dielectric Modeling}

Previous studies developed a model for the dielectric and conduction currents generated when a saturated rock was subjected to electromagnetic fields. This model was based on Archie's Rule for conduction and Sillars' model of an inhomogeneous dielectric. During February, a study of partially saturated rocks was initiated.

Partial saturation occurs when gas or oil replaces some of the pore water. The new model uses Archie's Rule of the form

$$
\sigma_{\text {rock }}=\left(\mathrm{QS}_{\mathrm{W}}\right) \sigma_{\mathrm{H}_{2}} \mathrm{O}
$$

to describe the conduction currents. The dielectrics were again based on Sillars' work, although a decision was required as to where the water was located in the overall pore structure when saturation was incomplete. In past work, the pore structure was modeled as a distribution of spheriods with different aspect ratios. Thus pores could be long and thin at one extreme, or spheres at the other.

If a partially saturated rock is water wet, capillary forces cause the water to seek out the pores that maximize its surface-to-volume ratio. That means, starting from dry rock, the long and thin pores would fill first. When this information is used in Sillars' model, the dielectric constant is strongly frequencydependent, even for small values of $S_{w}$. This prediction is quite compatible with the results of Soviet investigations. As a practical matter, it means that the dielectric data are not strong functions of $S_{w}$. Consequently, useful information as to the pore geometry, and hence the permeability, may be obtained without accurate knowledge of $\mathrm{S}_{\mathrm{w}}$. On the other hand, the conduction currents are more strongly dependent on $S_{W}$ and yield a measurement of its value.

Rough aspects of this work will be defined over the next few months.

\section{Laboratory Investigations}

The most complete study on the dielectric properties of rocks or rock-like materials is the work of Poley, Nooteboom, and de Waal (The Log Analyst, May-June 1978, p. 8). Their work was on "nearly clay-free sandstones," but the quantity of clay actually present was ill-defined. Since clays can have a significant and unknown effect on the dielectric as well as resistive behavior of inhomogeneous materials, work will be initiated on rock-like materials which are known to be clay free.

Two approaches were studied during February. The first considered the use of fritted 
glass disks which are porous materials commonly used for filtration. Inquiries were made to Corning Glass Works of the availability of disks of sufficiently large thickness, which would be advantageous since several investigators at Sandia, PRRC, Amoco, and Chevron could all study exactly the same material. The largest disk commonly manufactured by Corning is $60 \mathrm{~mm}$ in diameter but only $4 \mathrm{~mm}$ thick. This size is reasonable for the dielectric measurements but probably cannot be effectively used by the other investigators.

The second approach was to use a collection of packed spheres of very small diameter. This concept is interesting since the pore geometry is known exactly if the spheres are carefully packed into their densest configuration. Inquiries are being made of the availability of the appropriate spheres.

The possibility of constructing suitable ceramics of different porosities is also under investigation.

\section{Probe Modeling}

Computer programming efforts have completed three major tasks. The evaluation of Hankel functions with complex arguments has been improved to an accuracy of better than the sixth significant digit. After the accuracy was assured, numerical integrations were improved to perform 40 to 200 times faster. The fast convergence was from analytical transformations in the complex plane. The third major task was the completion of plotting subroutines for displaying contour curves.
Responses of coils in a borehole formation are generally expressed in terms of the refractive index which quantifies the wave-front propagation velocity and attenuation rate. The refractive index of a formation becomes the square root of complex dielectric constant when the magnetic permeability of a formation is assumed the same as that of an empty space. A set of universal curves has been completed to evaluate the refractive index of a formation when coil responses are known. For the purpose of interrogating all possible formations, three receiving coils are selected at 10,20 and 30 times the coil diameter from the transmitting coil. As soon as the normalized responses of these coils are measured, the formation parameters can be readily evaluated from three refractiveindex charts. The utility of these charts is being documented.

Effects of borehole fluids are being evaluated to quantify the extent of perturbation to coil responses. Preliminary results indicate that borehole effects are negligibly small if operating frequencies are low and formations are not too lossy. In general, borehole effects are quantifiable as long as the parametric variations can be assigned realistically. A plan to vary coil diameter with respect to borehole size is scheduled for March.

\section{Schedule Status}

A milestone chart depicting Sandia's WGSP progress is in Figure 4-11. 


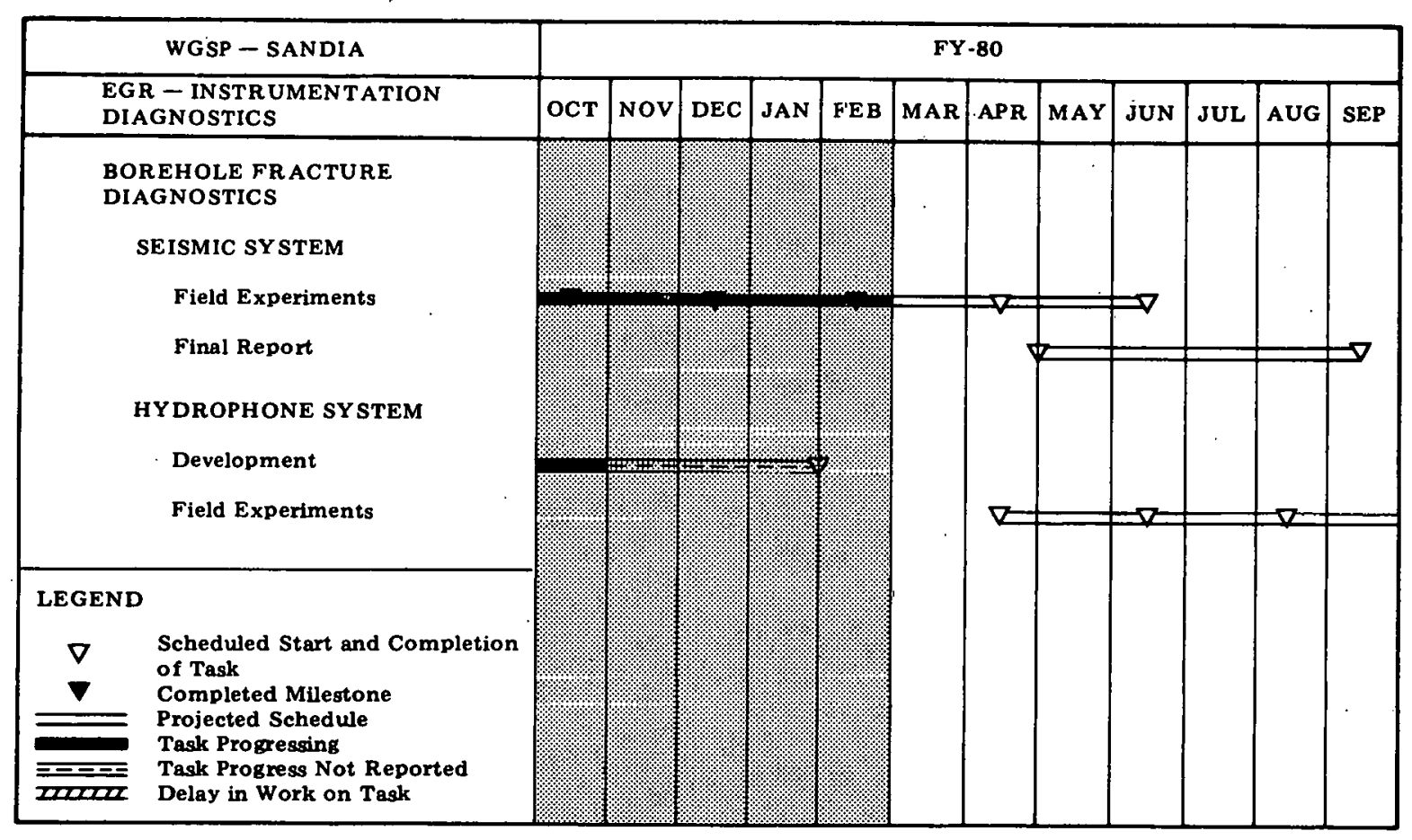

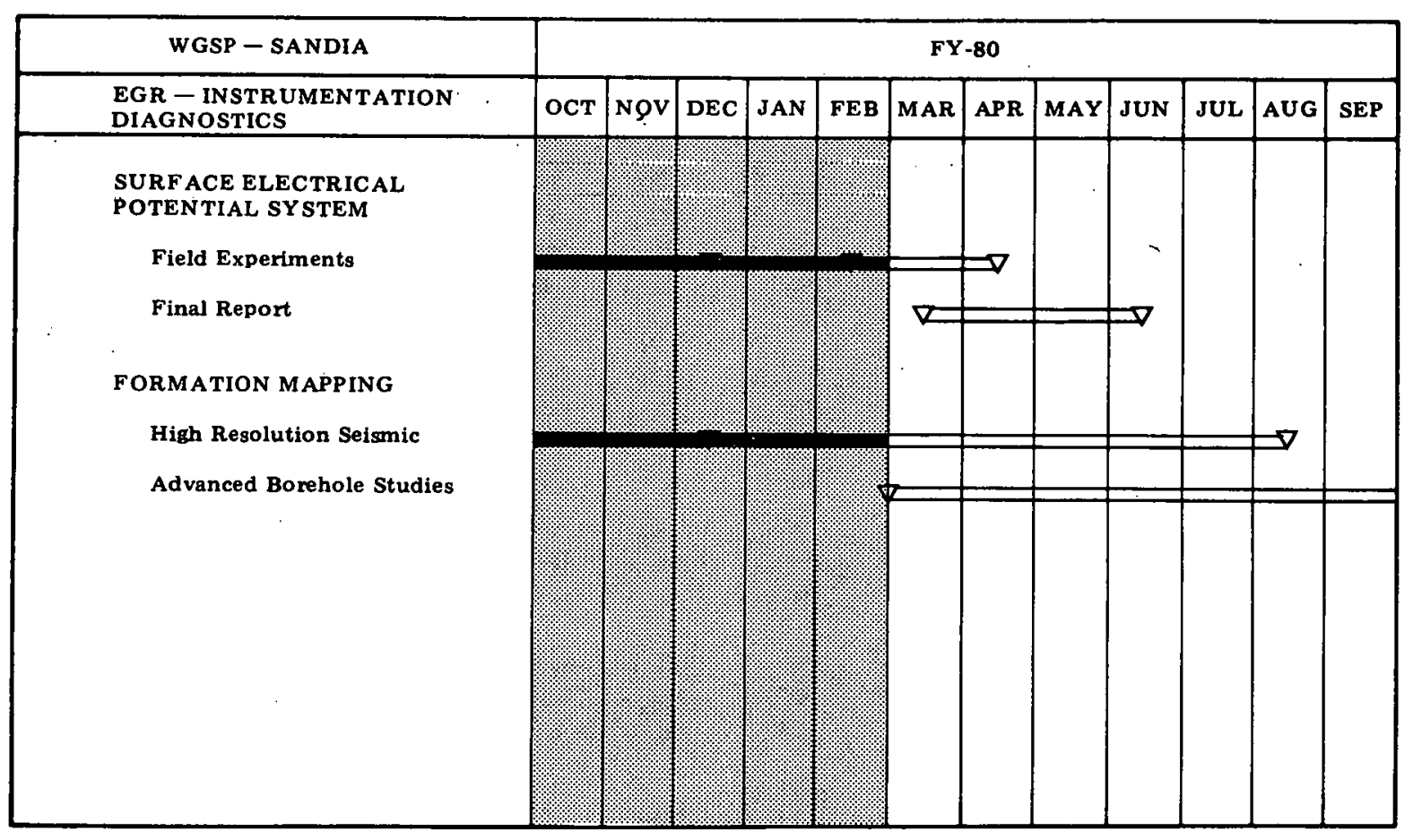

Figure 4-11 Milestone Chart - Sandia 


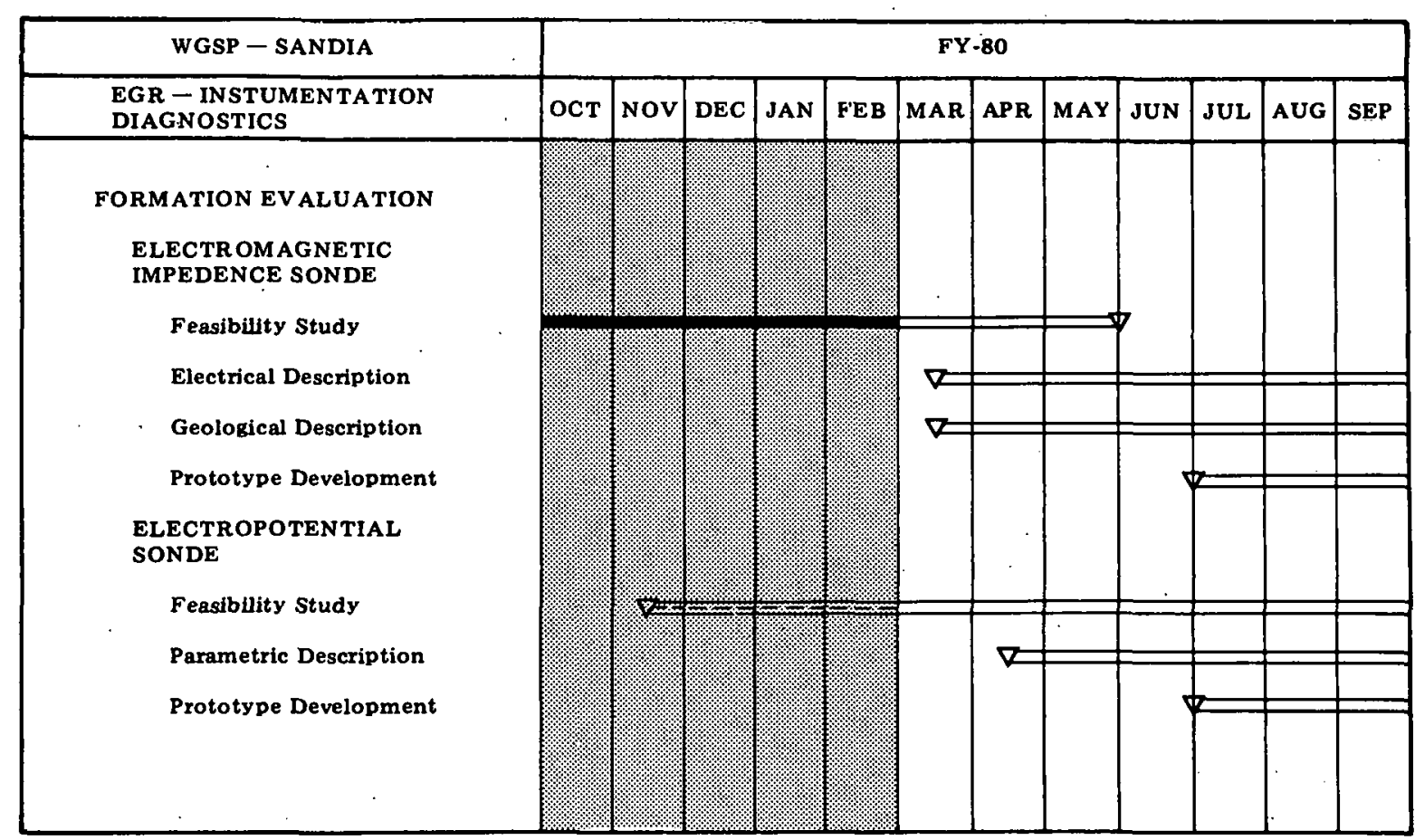

Figure 4-11 Continued 
THIS PAGE

\section{WAS INTENTIONALLY \\ LEFT BLANK}




\section{SECTION 5}

\section{FIELD TESTS \& DEMONSTRATIONS}

\section{BACKGROUND}

Field tests are essential to verify the findings of laboratory tests and modeling studies. The field test and demonstration program involves cooperation between industry and government and also interacts geologic studies with laboratory research and development. The following projects are active in the WGSP:
- a dry gas injection experiment in the Wattenberg Field, Colorado, by Colorado Interstate Gas Company,

- MHF demonstrations by Gas Producing Enterprises in the Uinta Basin, Utah,

- MHF treatment of the Cotton Valley Limestone Formation in Limestone County, Texas, by Mitchell Energy Corporation, and

- a mineback testing program by Sandia Laboratories.

Table 5-1 summarizes WGSP MHF treatments. Progress of these projects is presented in the following sections. 
Table 5-1 MHF Contract Location and Frac Data

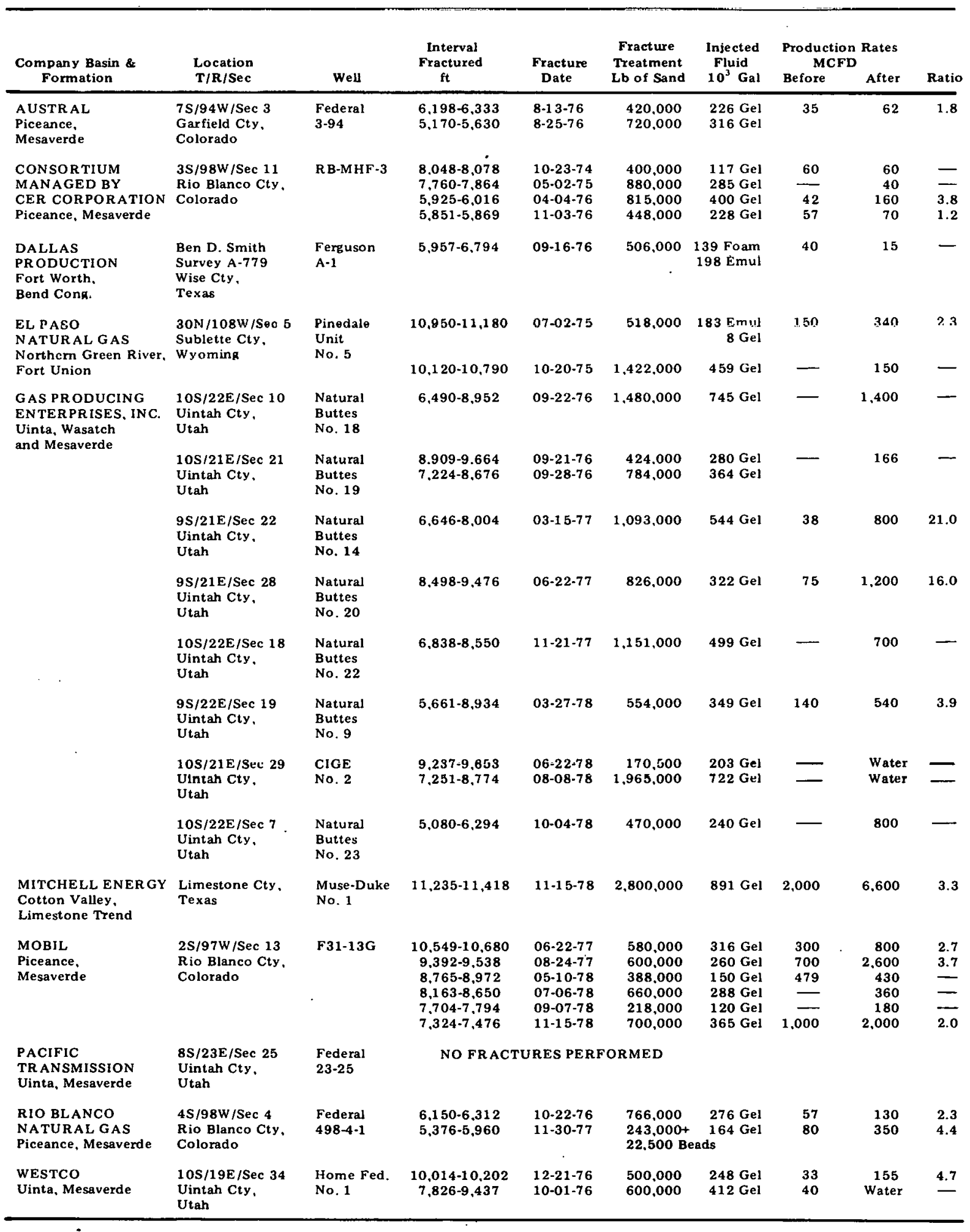


Contract Date:

Anticipated Completion Date:

Total Project Cost (estimated):

\section{September 1, 1977}

March 1, 1981

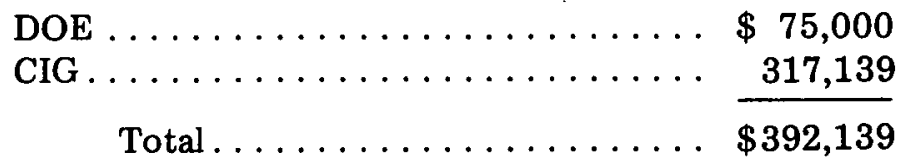

David Wilson

C. H. Atkinson

Principal Investigator:

\section{OBJECTIVE}

Cyclic injection of dry natural gas is the method to be used to increase production of tight gas sands.

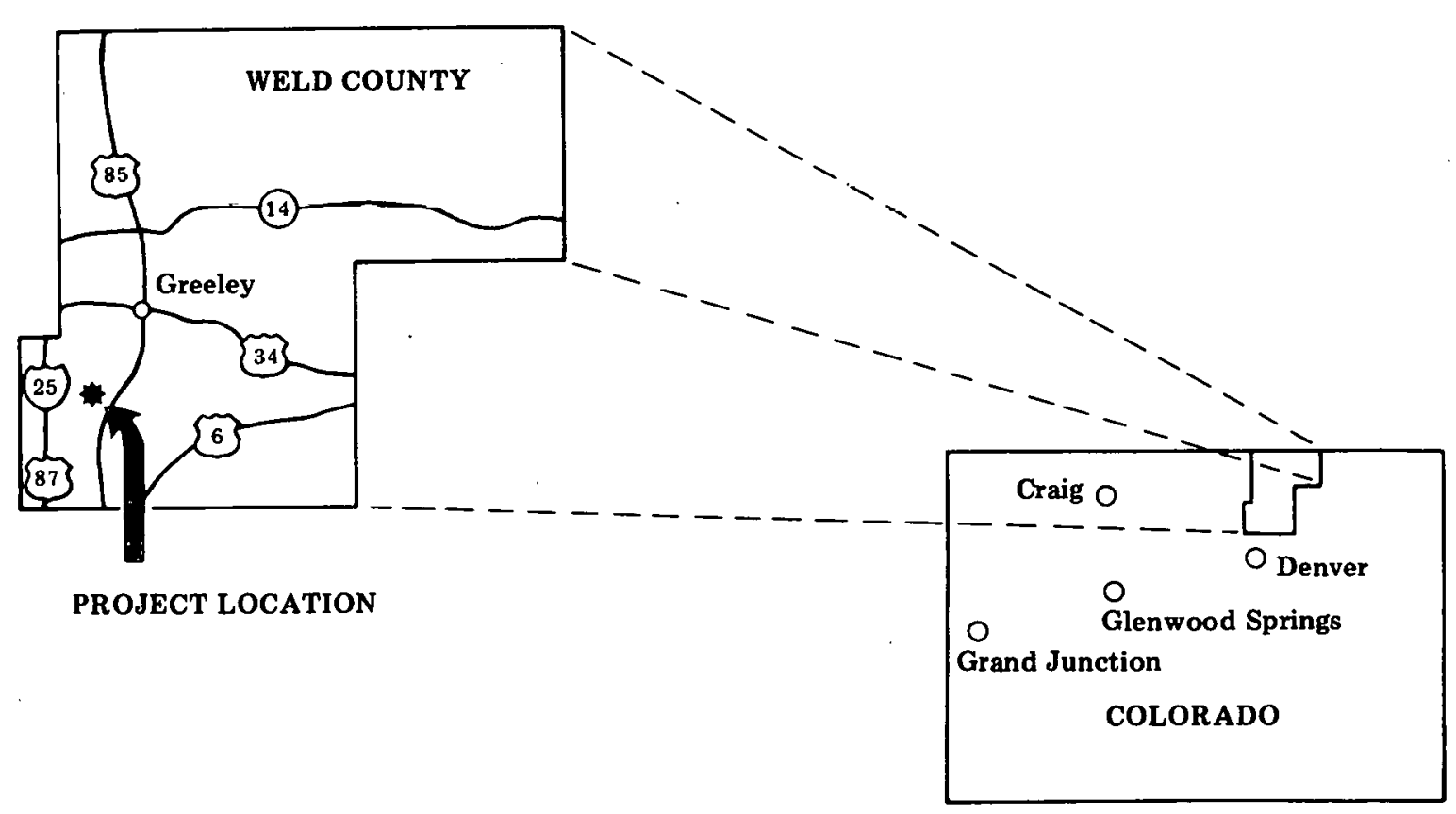




\section{COLORADO INTERSTATE GAS COMPANY}

Bottom-hole pressure measurement began February 1 at the Miller No. 1 well, and at the Sprague No. 1 well on February 19. On February 22, the BHP at the Miller No. 1 well was 1,260 psi, compared to the 1,115 psi achieved during the initial BHP measurement made prior to cyclic injection and withdrawl. 
CER Corporation

Las Vegas, Nevada

Principal Investigator:

Technical Advisor for DOE:
Status: Operational

R. L. Mann

C. H. Atkinson

\section{OBJECTIVE}

The DOE Well Test Facility, consisting of two vehicles, will provide a deep well instrumentation and investigation system to monitor and evaluate the productive potential of all types of wells.

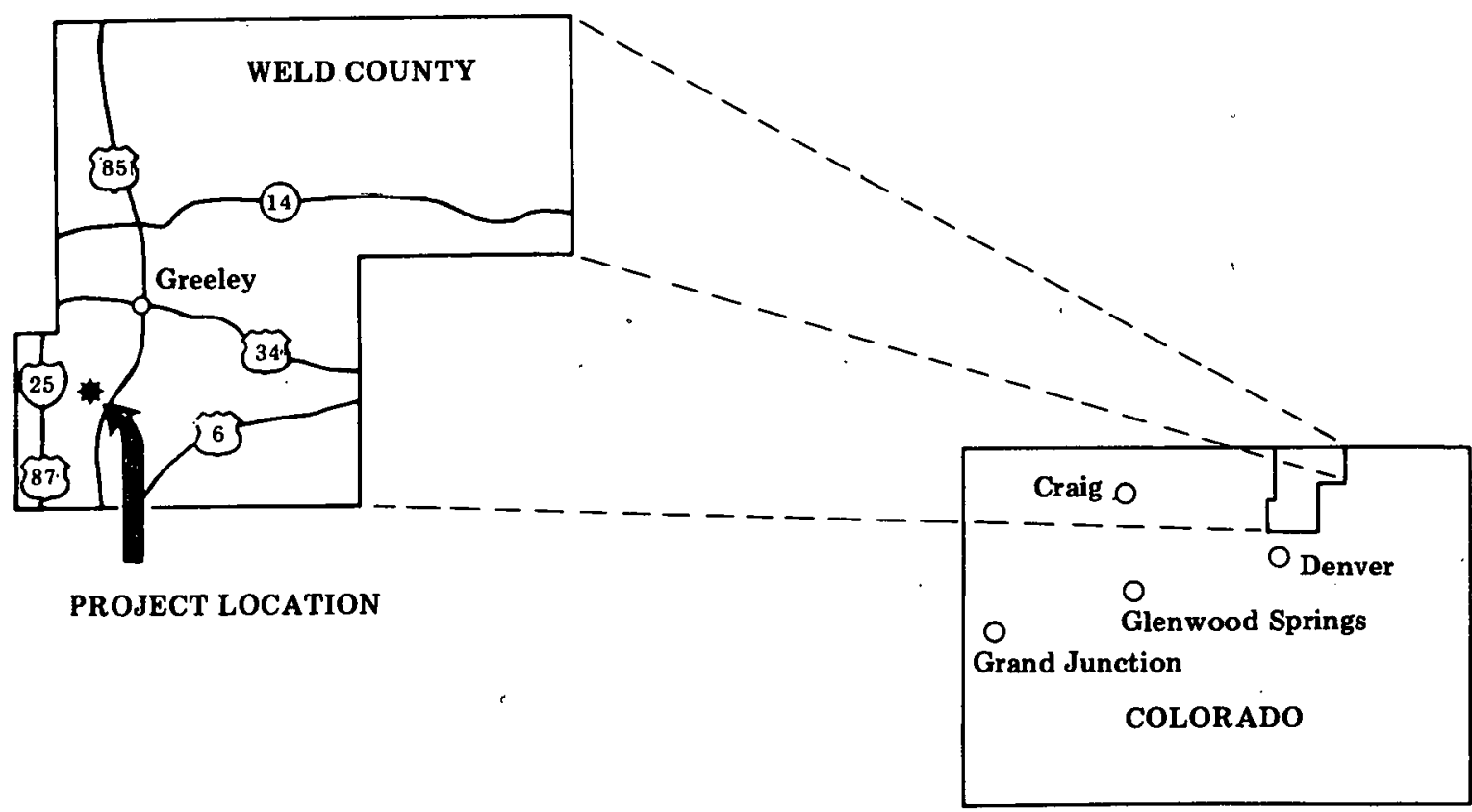




\section{DOE WELL TEST FACILITY}

The DOE Well Test Facility continued to provide support for the joint DOE/CIG cyclic dry gas injection experiment on two wells, Miller No. 1 and Sprague No. 1, Wattenberg Field, Colorado.

The test facility, located at the Miller No. 1 site, collected data during a drawdown test in late January. Several factors prohibited maintaining constant flow conditions during the drawdown. Table 5-2 is an abbreviated list of the drawdown data, and Figure 5-1 presents a graphical representation of the data. The flow rate was calculated using a standard orifice meter run, while bottom-hole pressure and temperature were measured with an H.P. pressure transducer and a GO temperature probe, respectively.
Miller No. 1 was shut in on January 30 and bottom-hole data were gathered continually during February. This buildup will continue through mid-March, when a modified isochronol test will be performed.

Table $5-3$ is an abbreviated list of the data gathered during the buildup. Figure $5-2$ is a log-log plot of the Miller bottom-hole pressure data. A slope of 1 is shown, which indicates afterflow or wellbore storage that seems to occur for about the first two hours. The slope of $1 / 2$ indicates linear flow, presumably through the fracture, and appears to continue for at least the first 100 hours.

The data will be analyzed and compared to previous test data. 
Table 5-2 Miller No. 1 Drawdown Data, January 18 - 30, 1980

\begin{tabular}{|c|c|c|}
\hline $\begin{array}{l}\text { Time } \\
\text { (Hrs) }\end{array}$ & $\begin{array}{c}\text { Flow } \\
\text { (MCFD) }\end{array}$ & $\begin{array}{c}\text { Bottomhole } \\
\text { Pressure } \\
\text { (psia) }\end{array}$ \\
\hline 0.00000 & 487.434 & $1,579.92$ \\
\hline 0.16667 & 515.999 & $1,565.65$ \\
\hline 0.33334 & 524.110 & $1,556.35$ \\
\hline 0.50000 & 507.867 & $1,548.55$ \\
\hline 0.66667 & 497.444 & $1,542.23$ \\
\hline 0.83334 & 493.484 & $1,536.81$ \\
\hline 1.00000 & 486.536 & $1,531.88$ \\
\hline 1.33334 & 488.736 & $1,523.00$ \\
\hline 1.50000 & 489.744 & $1,518.64$ \\
\hline 1.83334 & 507.775 & $1,509.83$ \\
\hline 2.00000 & 501.919 & $1,505.71$ \\
\hline 2.33334 & 507.900 & $1,497.90$ \\
\hline 2.50000 & 499.451 & $1,494.38$ \\
\hline 2.83334 & 430.627 & $1,490.45$ \\
\hline 3.00000 & 396.727 & $1,489.47$ \\
\hline 3.33334 & 363.086 & $1,499.78$ \\
\hline 3.50000 & 384.986 & $1,492.21$ \\
\hline 3.83334 & 289.071 & $1,497.67$ \\
\hline 4.00000 & 325.288 & $1,495.92$ \\
\hline 4.50000 & 257.310 & $1,494.43$ \\
\hline 5.01667 & 271.599 & $1,491.86$ \\
\hline 5.53334 & 336.482 & $1,487.37$ \\
\hline 10.60000 & 370.217 & $1,455.19$ \\
\hline 16.10000 & 311.377 & $1,427.77$ \\
\hline 21.10000 & 347.165 & $1,399.04$ \\
\hline 26.10000 & 299.182 & $1,383.22$ \\
\hline 31.10000 & 293.196 & $1,362.76$ \\
\hline 36.10000 & 363.774 & $1,349.32$ \\
\hline 41.60000 & 342.706 & $1,326.91$ \\
\hline 51.60000 & 311.885 & $1,314.41$ \\
\hline 64.60001 & 319.293 & $1,255.42$ \\
\hline 74.60001 & 488.456 & $1,179.84$ \\
\hline 84.60001 & 581.933 & $1,065.77$ \\
\hline 99.10000 & 541.742 & $1,016.93$ \\
\hline 104.10000 & 529.060 & 979.64 \\
\hline 114.60000 & 501.063 & 918.76 \\
\hline 124.60000 & 842.076 & 657.84 \\
\hline 135.10000 & 824.231 & 427.91 \\
\hline 150.60000 & 693.759 & 210.27 \\
\hline 166.10000 & 586.610 & 171.08 \\
\hline 176.10000 & 549.934 & 160.08 \\
\hline 191.60000 & 495.489 & 150.48 \\
\hline 201.60000 & 474.605 & 152.25 \\
\hline 245.60000 & 587.263 & 203.17 \\
\hline 250.60000 & 433.303 & 166.27 \\
\hline 261.10000 & 359.722 & 144.68 \\
\hline 271.10000 & 314.892 & 144.13 \\
\hline 281.60000 & 262.350 & 145.72 \\
\hline 287.73330 & 280.724 & 139.17 \\
\hline
\end{tabular}



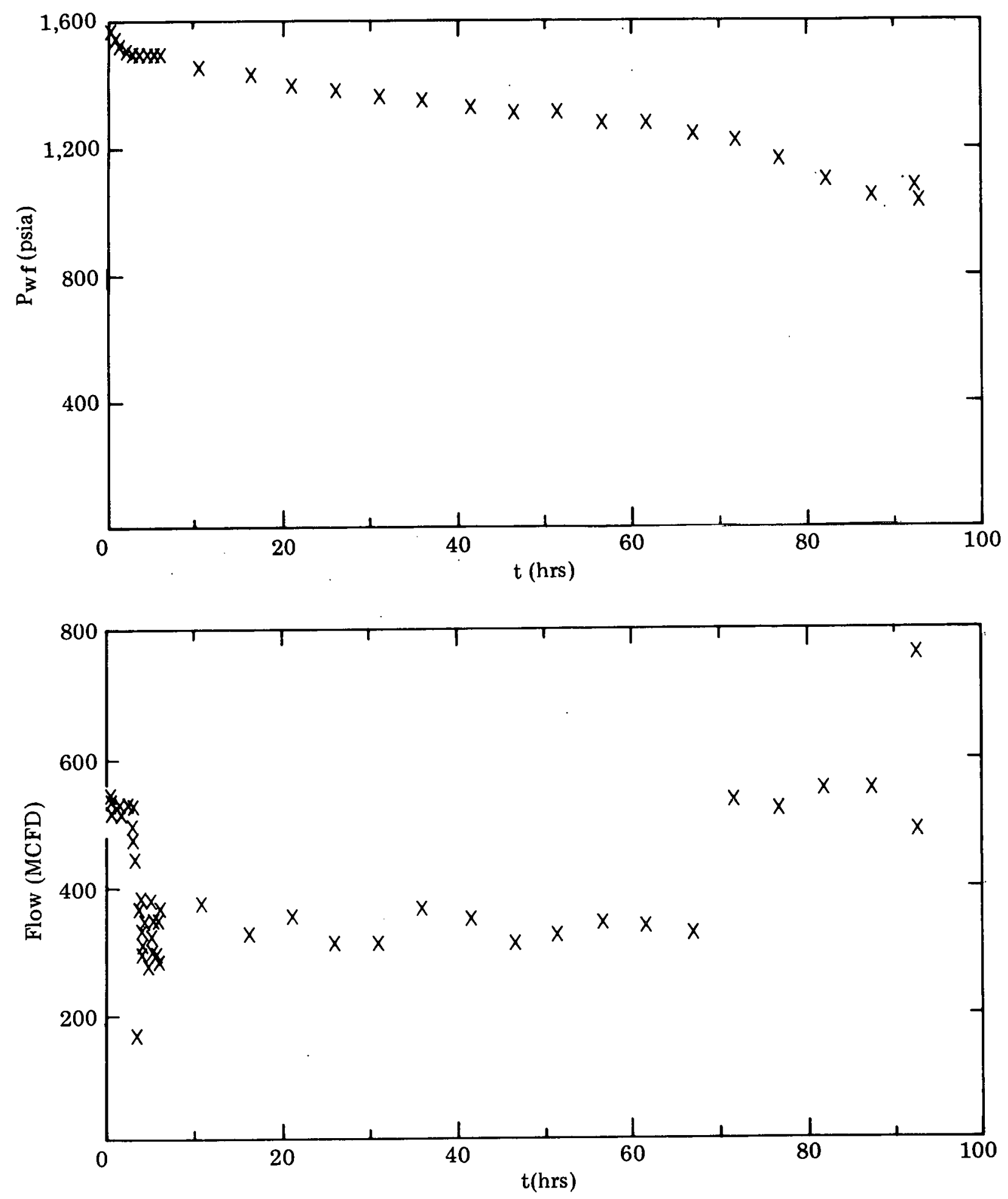

Figure 5-1 Pressure and Flow Data from Miller No. 1 Drawdown Test 
Table 5-3 Miller No. 1 Bottomhole Pressure Buildup Test, January 30 - February 29, 1980

\begin{tabular}{|c|c|c|c|}
\hline $\begin{array}{c}\text { Shut In } \\
\text { Time } \\
\text { (hrs) }\end{array}$ & $\begin{array}{c}\text { Pressure } \\
\text { (psi) }\end{array}$ & $\begin{array}{c}\text { Shut In } \\
\text { Time } \\
\text { (hrs) }\end{array}$ & $\begin{array}{c}\text { Pressure } \\
\text { (psi) }\end{array}$ \\
\hline .04 & 151.4 & 18.17 & 646.8 \\
\hline .08 & 162.8 & 20.33 & 668.4 \\
\hline .13 & 173.5 & 24.50 & 705.1 \\
\hline .19 & 183.6 & 27.50 & 728.3 \\
\hline .28 & 193.9 & 30.50 & 749.2 \\
\hline .41 & 205.4 & 34.50 & 774.1 \\
\hline .53 & 216.0 & 36.50 & 785.4 \\
\hline .63 & 226.1 & 40.50 & 806.6 \\
\hline .76 & 237.0 & 45.50 & 830.4 \\
\hline .83 & 247.4 & 50.50 & 851.9 \\
\hline .94 & 257.5 & 56.50 & 874.8 \\
\hline 1.10 & 267.7 & 63.00 & 896.8 \\
\hline 1.43 & 288.4 & 70.00 & 917.9 \\
\hline 1.59 & 298.4 & 74.00 & 929.1 \\
\hline 1.78 & 308.7 & 82.50 & 950.5 \\
\hline 2.17 & 328.8 & 87.00 & 960.8 \\
\hline 2.62 & 349.4 & 93.55 & 981.8 \\
\hline 2.86 & 359.6 & 117.55 & $1,004.0$ \\
\hline 3.40 & 380.4 & 157.55 & $1,046.0$ \\
\hline 3.98 & 400.7 & 189.55 & $1,078.0$ \\
\hline 4.30 & 410.9 & 209.55 & $1,099.0$ \\
\hline 4.98 & 431.2 & 233.55 & $1,119.0$ \\
\hline 5.73 & 451.5 & 262.55 & $1,140.0$ \\
\hline 6.58 & 472.1 & 279.55 & $1,150.0$ \\
\hline 7.03 & 482.2 & 297.55 & $1,160.0$ \\
\hline 7.50 & 492.2 & 337.55 & $1,181.0$ \\
\hline 7.98 & 502.2 & 385.20 & $1,201.0$ \\
\hline 9.17 & 524.6 & 413.20 & $1,212.0$ \\
\hline 9.83 & 536.4 & 443.20 & $1,222.0$ \\
\hline 10.50 & 547.5 & 485.26 & $1,232.0$ \\
\hline 13.50 & 591.7 & 519.26 & $1,242.0$ \\
\hline 15.17 & 612.9 & 559.26 & $1,252.0$ \\
\hline
\end{tabular}


$\stackrel{\infty}{\infty}$

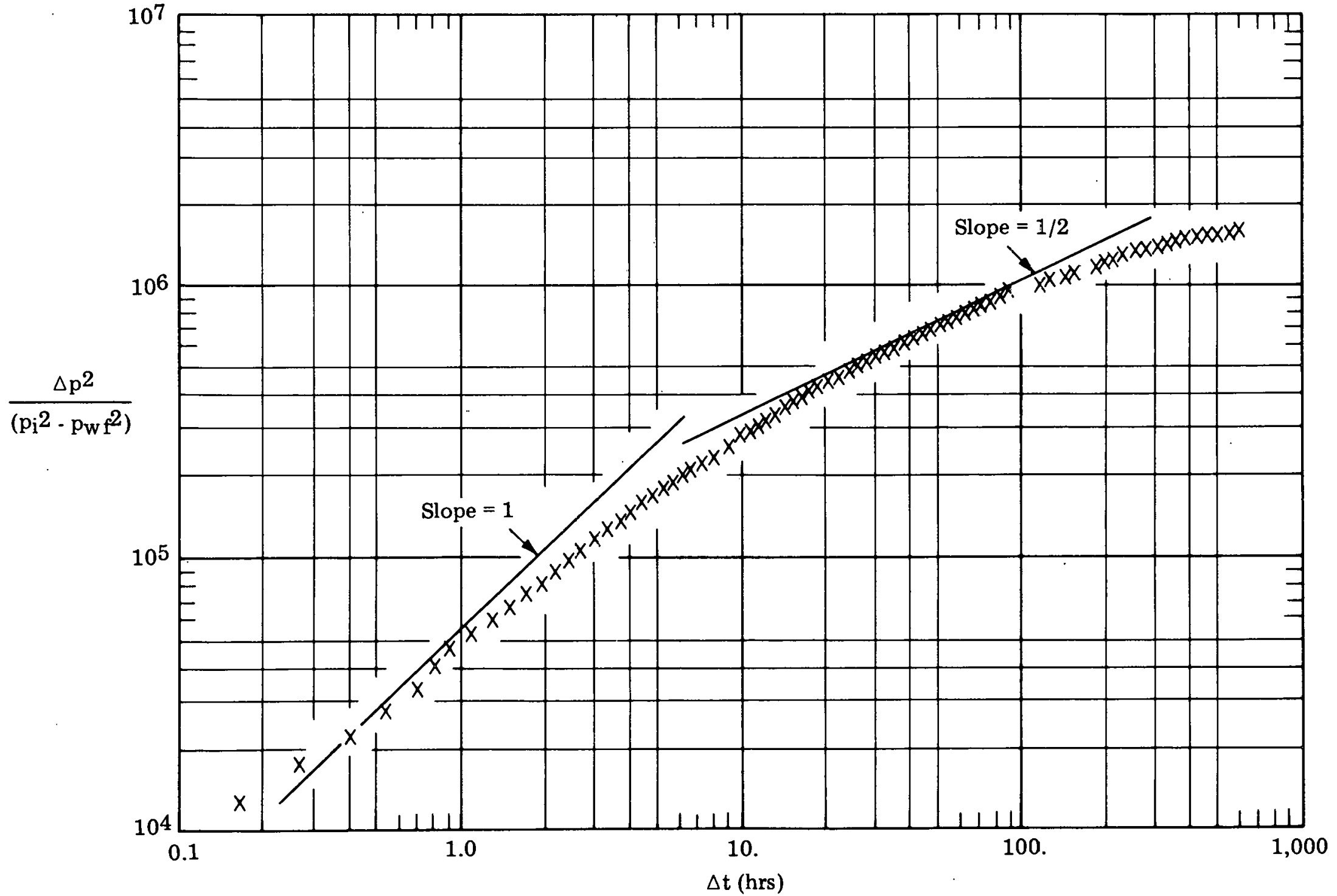

Figure 5-2 Miller No. 1 Pressure Buildup Data, January 30 - February 29, 1980 
NATURAL BUTTES UNIT, UINTAH COUNTY, DE-AC08-76 ET 12060 UTAH MASSIVE HYDRAULIC FRACTURING

\section{DEMONSTRATION}

Gas Producing Enterprises, Inc.

Status: Active

Subsidiary of Coastal States Gas Co.

Houston, Texas

Contract Date:

July 1,1976

Anticipated Completion:

March 31, 1980

Total Project Cost (estimated):

DOE $\ldots \ldots \ldots \ldots \ldots \ldots \ldots \ldots \ldots 2,827,000$

Industry (prior costs) $\ldots \ldots \ldots \ldots \ldots \quad 1,881,000$

Industry (new costs) $\ldots \ldots \ldots \ldots \ldots \ldots \quad 3,051,000$

Total . . . . . . . . . . $\overline{\$ 7,759,000}$

Principal Investigator:

Technical Project Officer for DOE:

W. E. Spencer

C. H. Atkinson

\section{OBJECTIVE}

To evaluate the effectiveness of massive hydraulic fracturing for stimulating natural gas production from thick, deep sandstone reservoirs having low-permeability.

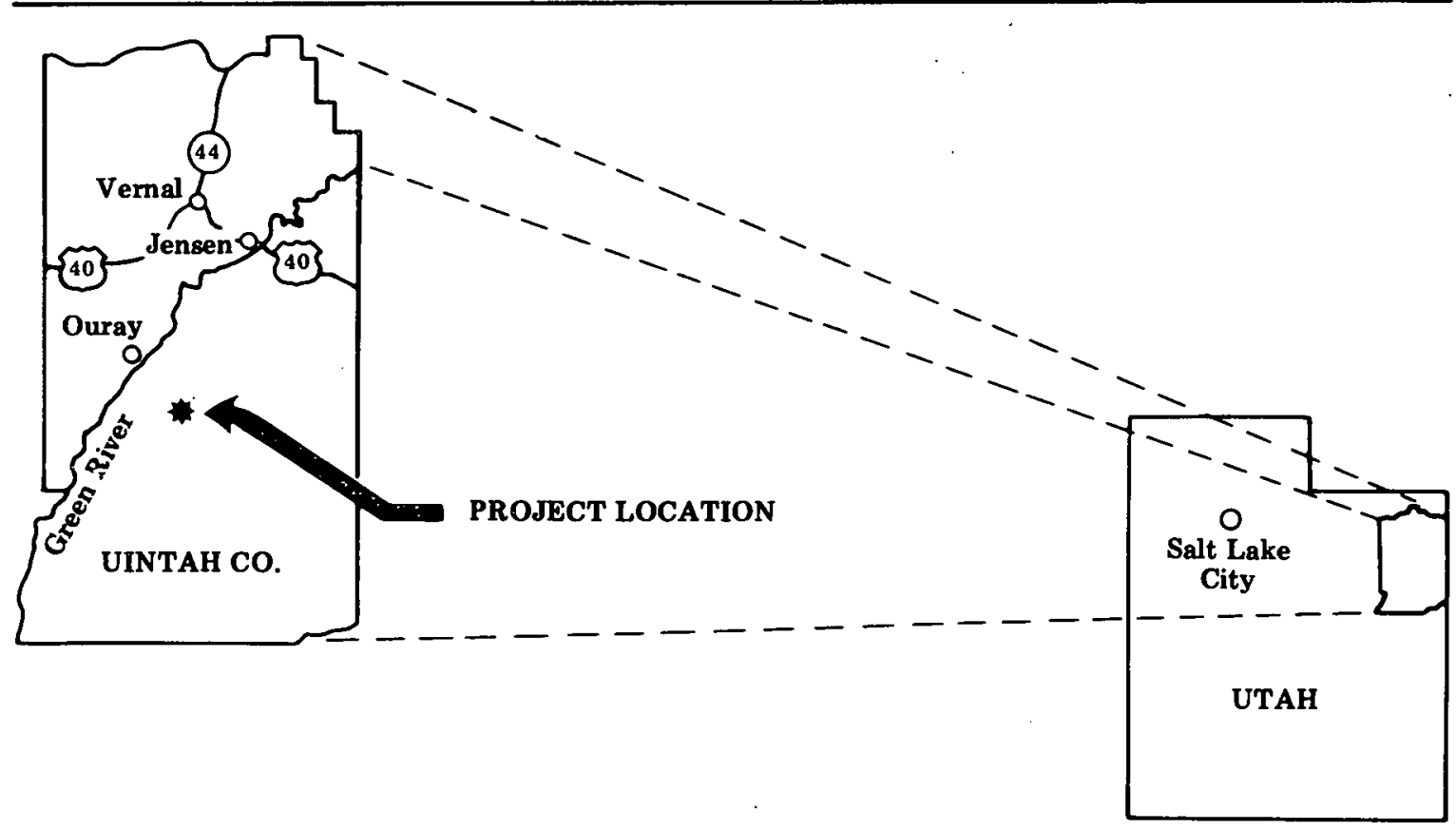




\section{GAS PRODUCING ENTERPRISES}

GPE's Natural Buttes Unit Nos. 9, 14, 18 and 20 flowed to sales during February with a total 51,960 MCF. NBU Nos. 19 and 22 were shut in. Figures 5-3 through 5-8 show the production histories of these wells.

A comprehensive final report is in preparation. 


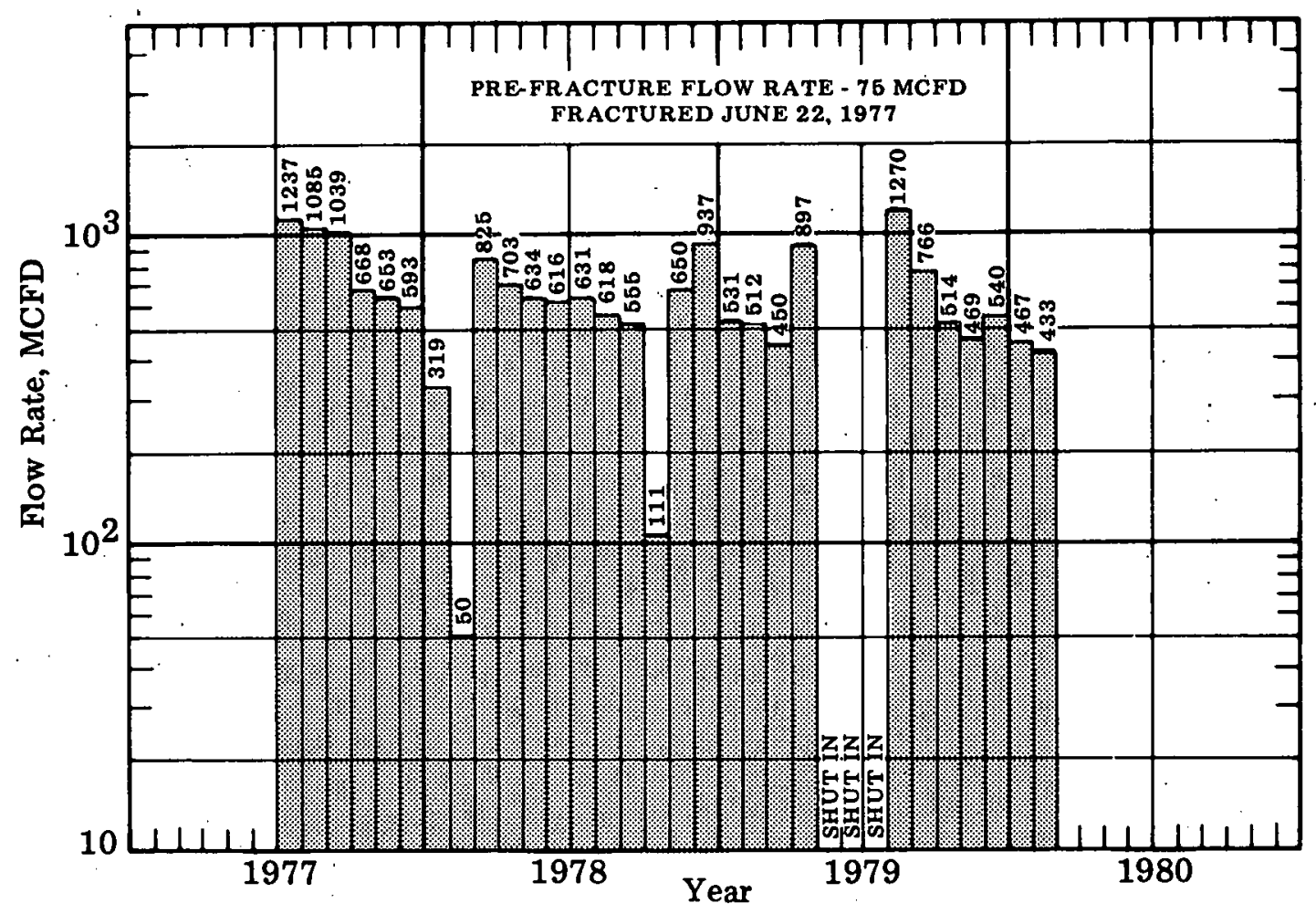

Figure 5-3 Flow Rate Performance of Natural Buttes No. 9 Well

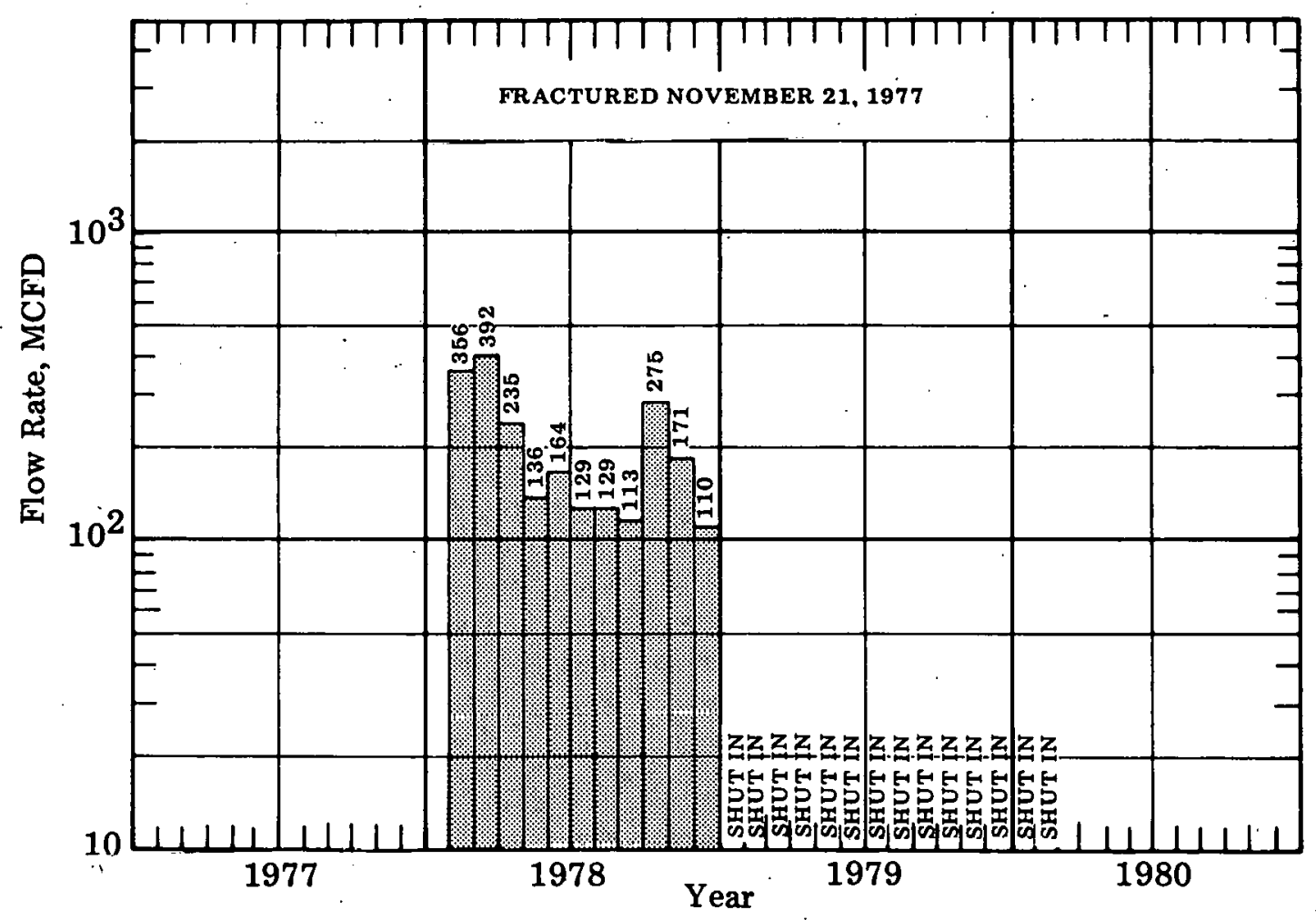

Figure 5-4 Flow Rate Performance of Natural Buttes No. 14 Well 


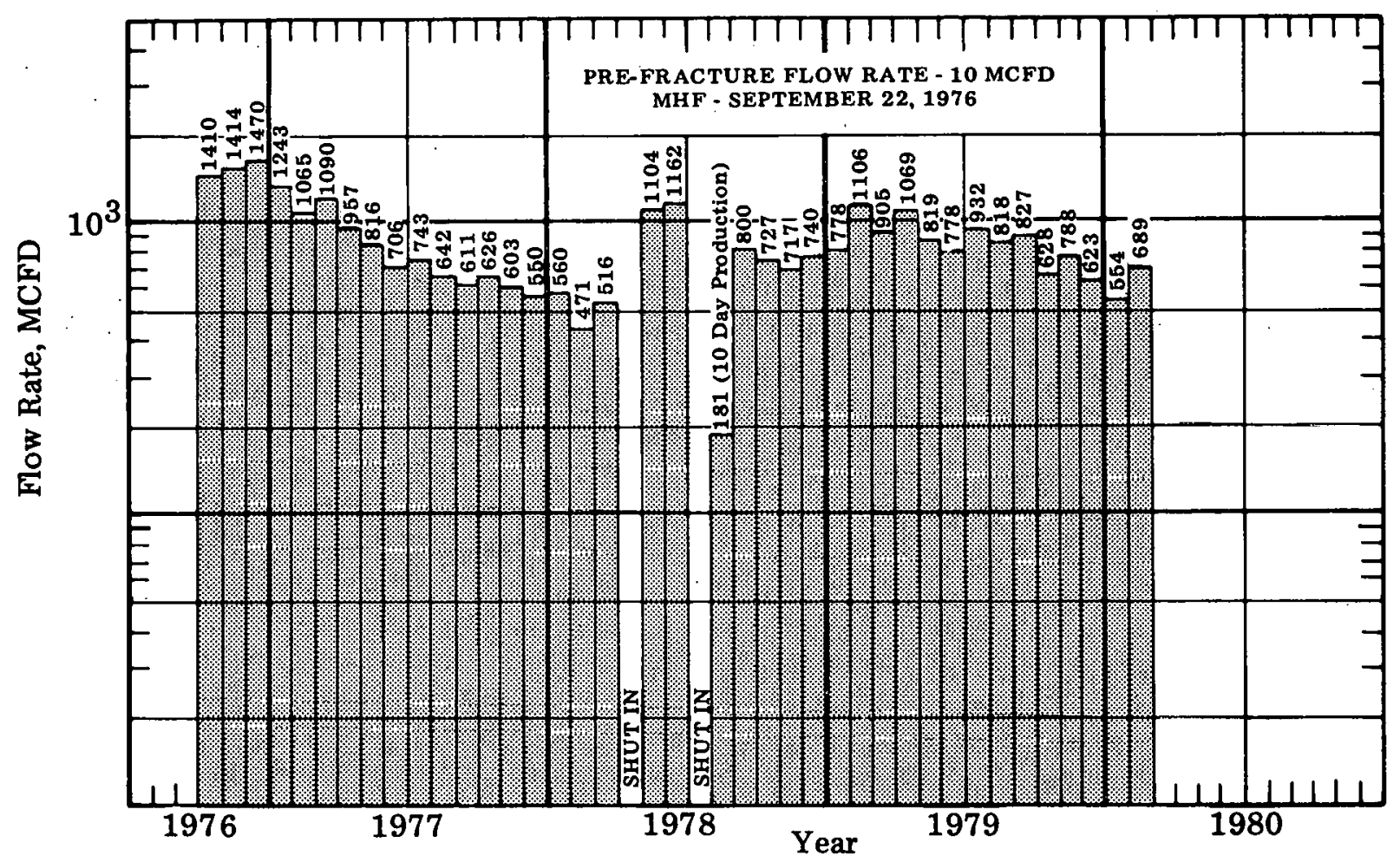

Figure 5-5 Flow Rate Performance of Natural Buttes No. 18 Well

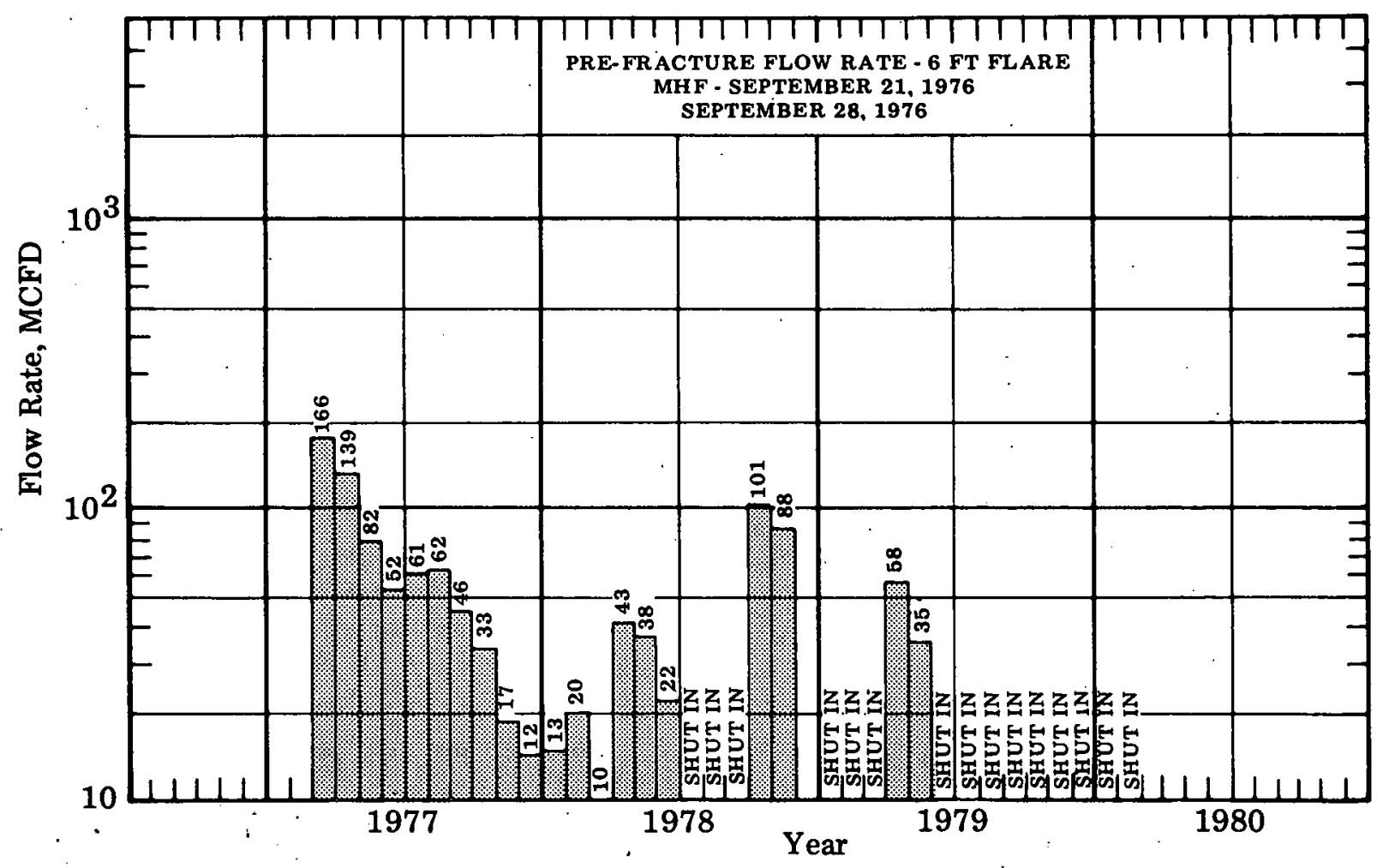

Figure 5-6 Flow Rate Performance of Natural Buttes No. 19 Well 


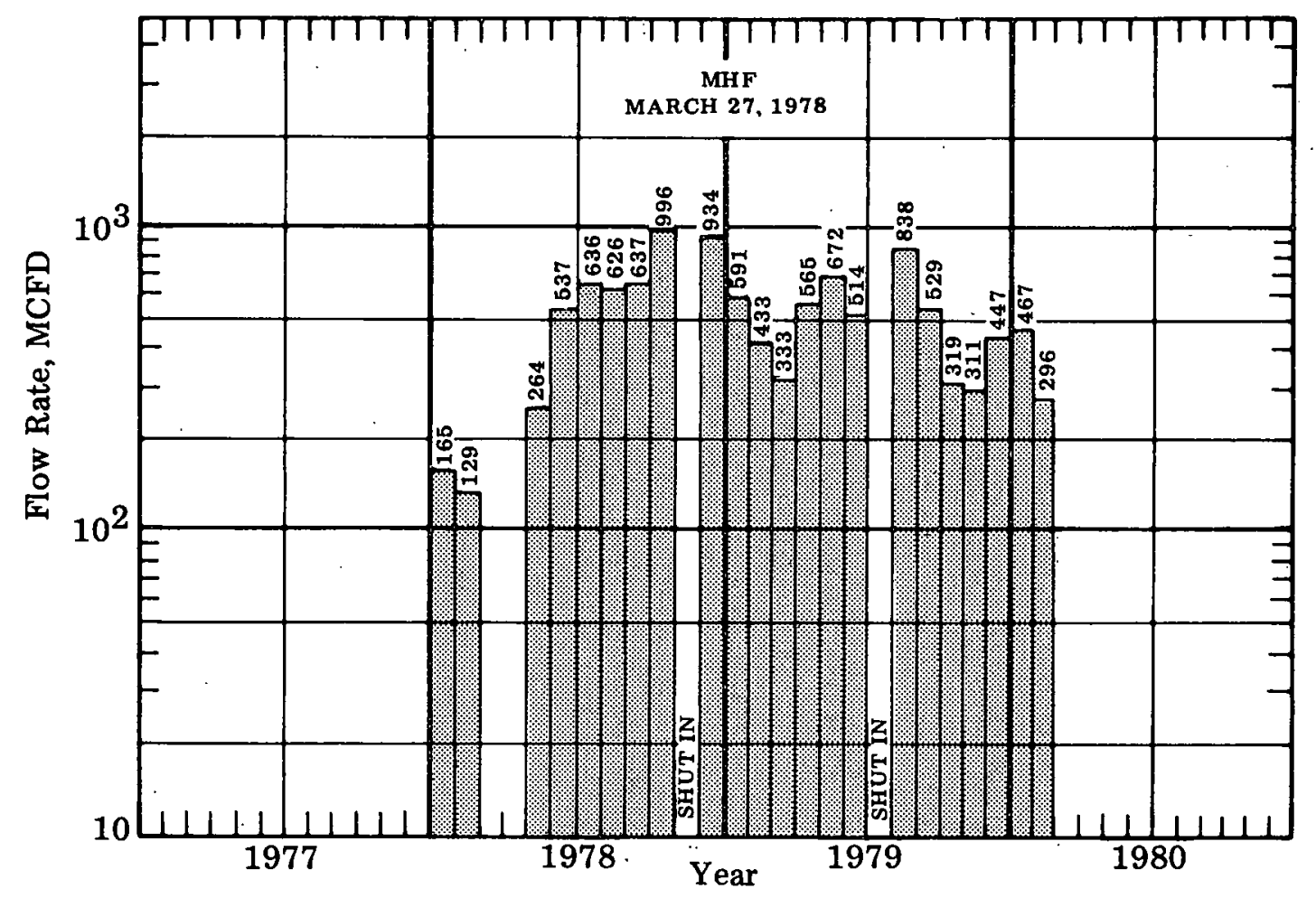

Figure 5-7 Flow Rate Performance of Natural Buttes No. 20 Well

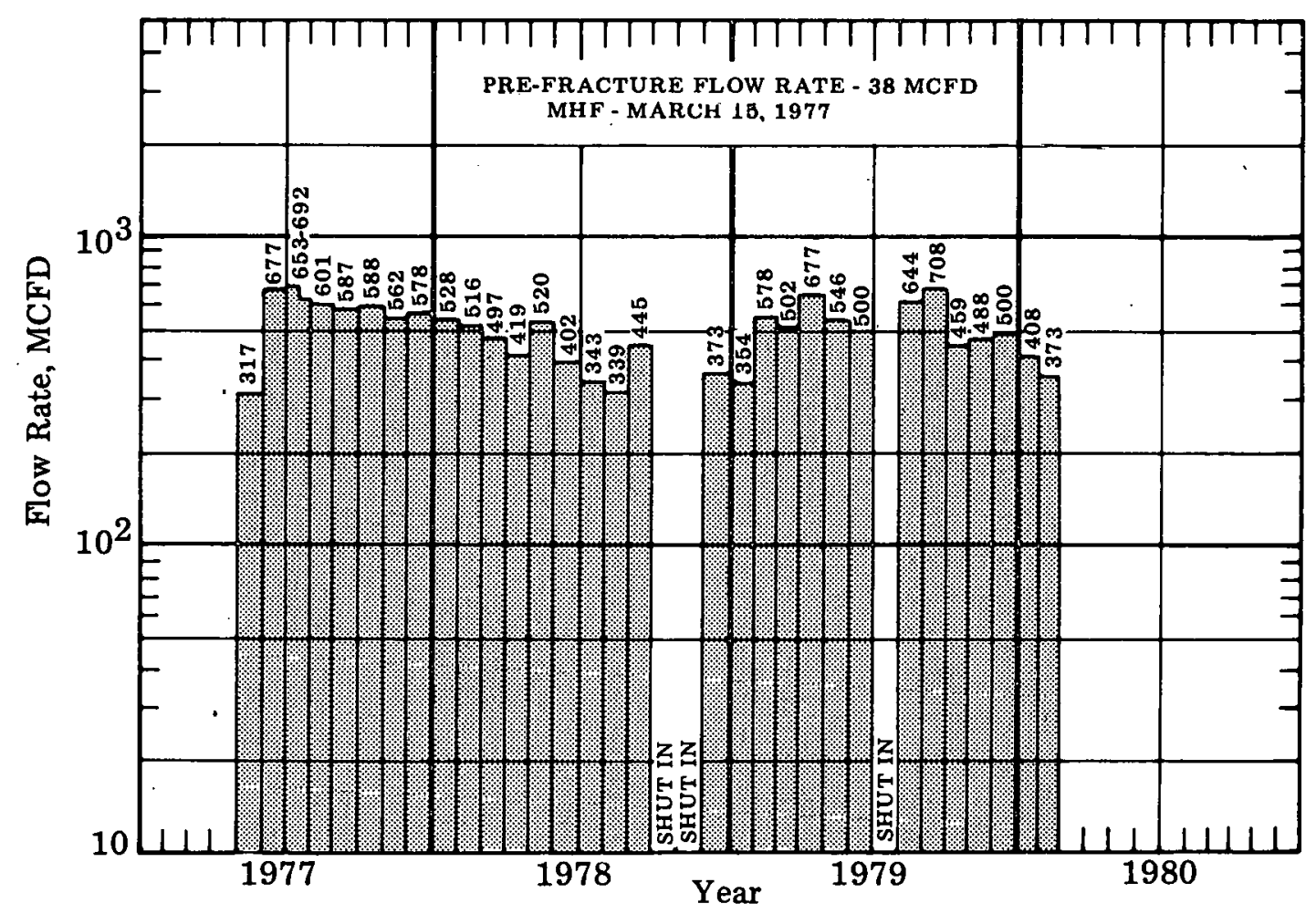

Figure 5-8 Flow Rate Performance of Natural Buttes No. 22 Well 
FALLON-NORTH PERSONVILLE FIELD,

DE - AC08 - 78ET12150 TEXAS, MASSIVE HYDRAULIC FRACTURING

\section{DEMONSTRATION}

Mitchell Energy Corporation

Status: Active

Houston, Texas

Contract Date:

Anticipated Completion:

March 15, 1978

April 30, 1979 (Extension being processed)

Total Project Cost (estimated):

DOE . . . . . . . . . . . . . $\$ \$ 553,771$

Industry $\ldots \ldots \ldots \ldots \ldots \ldots \ldots, 1,074,550$

Total. . . . . . . . . . . . $\$ 1,628,321$

Principal Investigator:

F. D. Covey

Technical Project Officer for DOE:

C. H. Atkinson

\section{OBJECTIVE}

To test massive hydraulic fracturing in the Cotton Valley Limestone Formation.

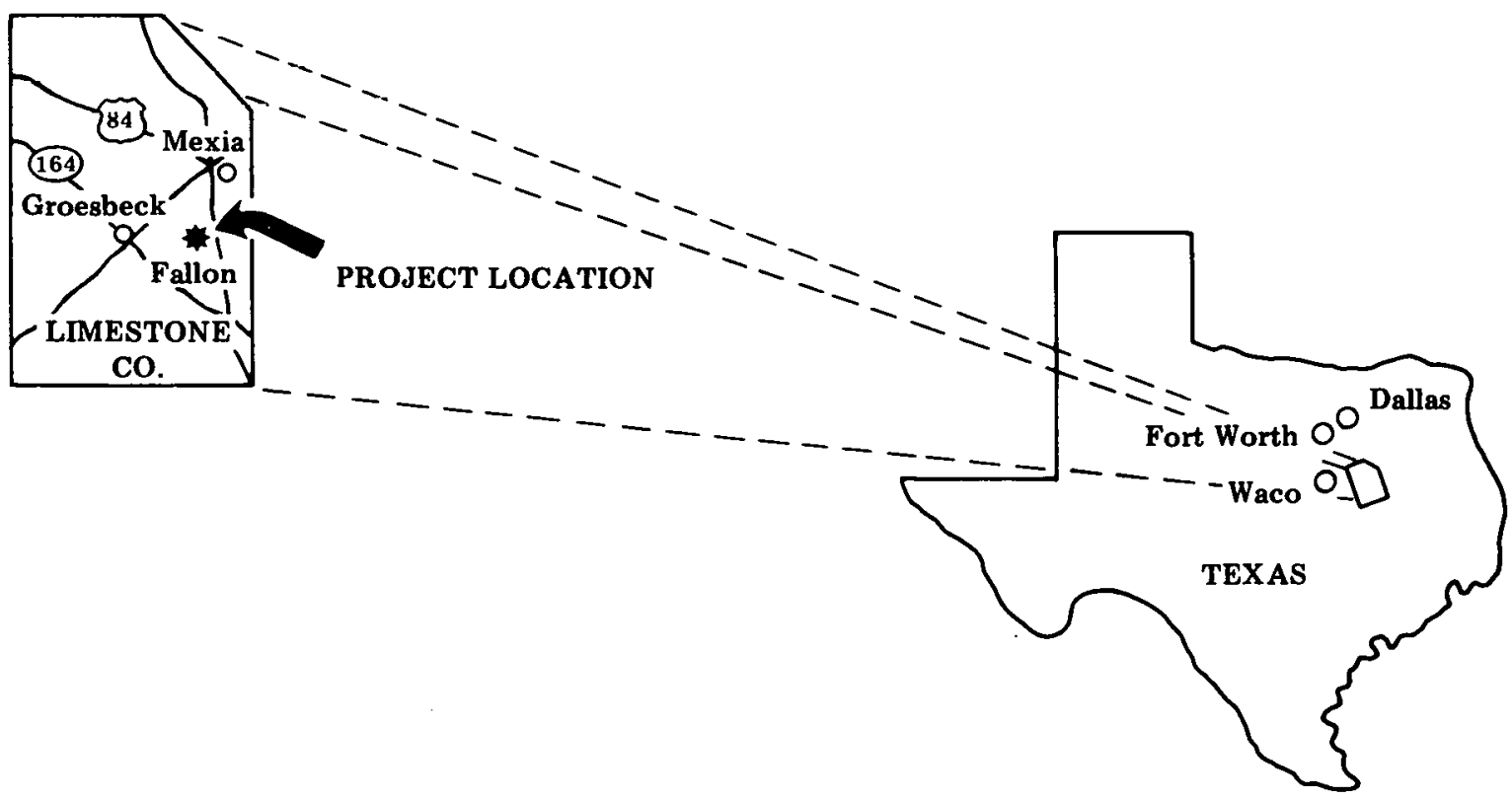


MITCHELL ENERGY CORPORATION

The final report is in preparation. 


\section{NEVADA TEST SITE \\ NYE COUNTY, NEVADA \\ MINEBACK TESTING}

Sandia Laboratories

Status: Active

Albuquerque, New Mexico

Principal Investigator:

D. A. Northrop

\section{OBJECTIVE}

To develop an understanding of the fracturing process for stimulation and thereby improve the production of natural gas from low-permeability reservoirs. This will be accomplished by conducting controlled fracture experiments which are accessible by mineback for direct observation and evaluation.

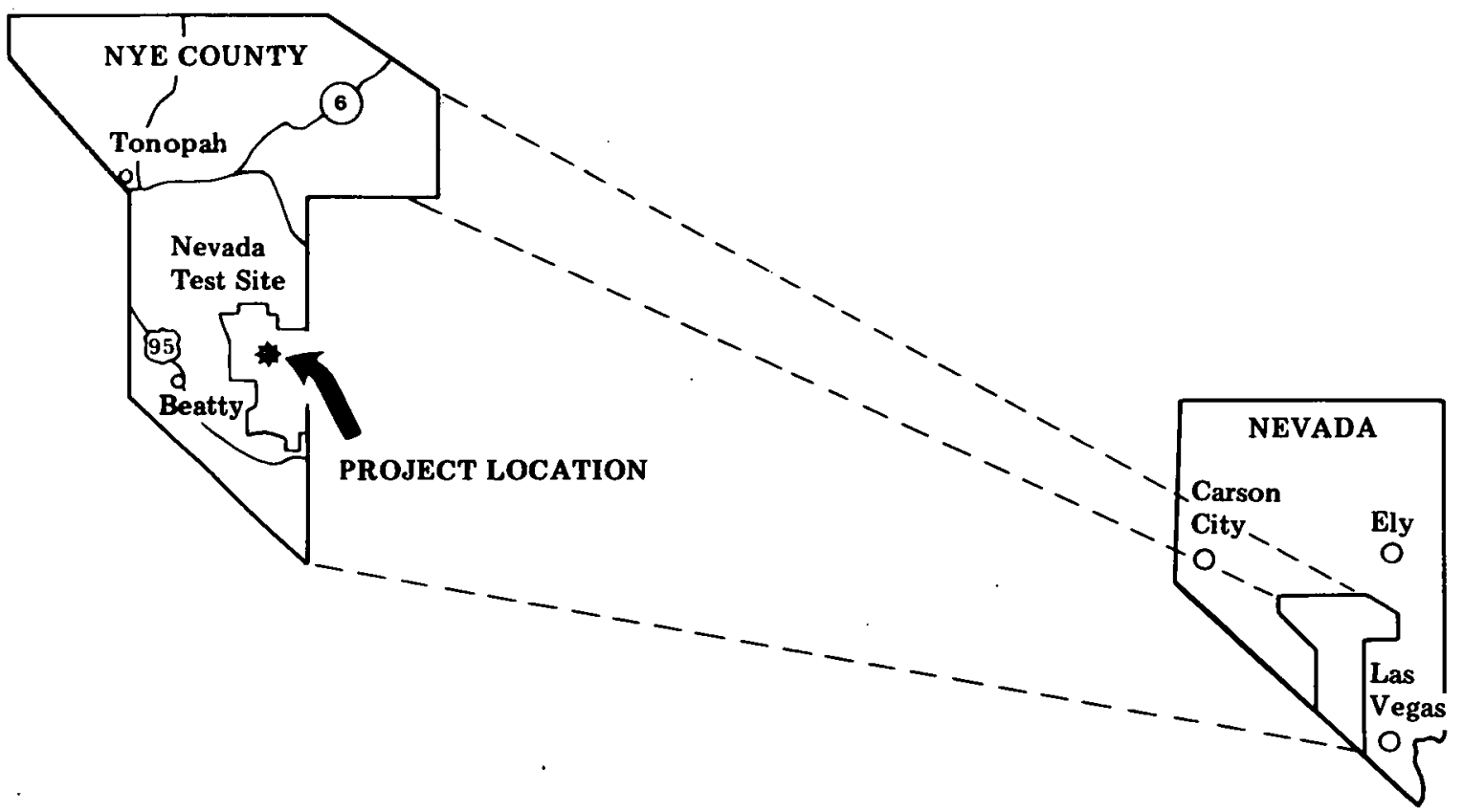


SANDIA - MINEBACK

\section{Hydraulic Fracturing Containment Experiments}

Hole No. 6 Formation Interface Fracture Experiment

Twenty-six coreholes have been completed including EV6.34 in February, shown in Figure 5-9. Approximately three holes will be drilled 20 to $40 \mathrm{ft}$ below the mineback drift to determine crack widths in the center of the fracture. There will be an attempt to draw width contours with this data, which will aid in the post-treatment analysis and understanding of the causes of the observed fracture geometry.

\section{Interface Test Series}

The Interface Test Series, a set of small hydraulic fracturing experiments conducted at the Nevada Test Site (NTS) to examine the factors which affect containment, has now been completed. These experiments showed that a material property difference is not an important factor in containing hydraulic fractures. However, it was evident that in situ stresses offer a predominant influence on fracture propagation, and regions of higher in situ stress will arrest fracture growth, and consequently contain a fracture.

A paper titled "In Situ Stresses: The Predominant Influence on Hydraulic Fracture Containment" is currently being prepared for the 1980 SPE/DOE Symposium on Unconventional Gas Recovery, May 18-21, in Pittsburgh, Pennsylvania. This paper integrates the above results with the results of previous experiments to show that a variation in the minimum principal in situ stress, whether due to faults, stratigraphy or tectonics, is the only factor which will have a significant effect on containment.

\section{Proppant Experiments}

A site is being prepared for proppant experiments and an in-tunnel pump system is under construction which will pump high viscosity gels with concentrations of sand up to $3 \mathrm{lbs}$ per gallon. These experiments are expected to commence in June.

\section{Rock Mechanics}

A field and laboratory program was recently initiated to determine the extent of influence of residual stress on hydraulic fracture growth in tuff at NTS. Residual strains in a large block of ashfall tuff from G-tunnel, containing a grout-filled fracture, have been measured in the laboratory by a double overcoring technique. Residual stresses calculated from these measurements show that the minimum horizontal principal compressive stress is within $10^{\circ}$ of being perpendicular to the hydraulic fracture plane. The stress magnitude difference of the principal residual stresses is $0.86 \mathrm{MPa}$ (compressive). The next step in this program will be overcoring measurements at several horizons adjacent to mined-back hydraulic fractures in G-tunnel to determine the in situ stress. The core will then be removed and overcored to obtain only the residual strain.

\section{Schedule Status}

Figure 5-10 is a milestone chart showing Sandia Mineback progress within the WGSP. 


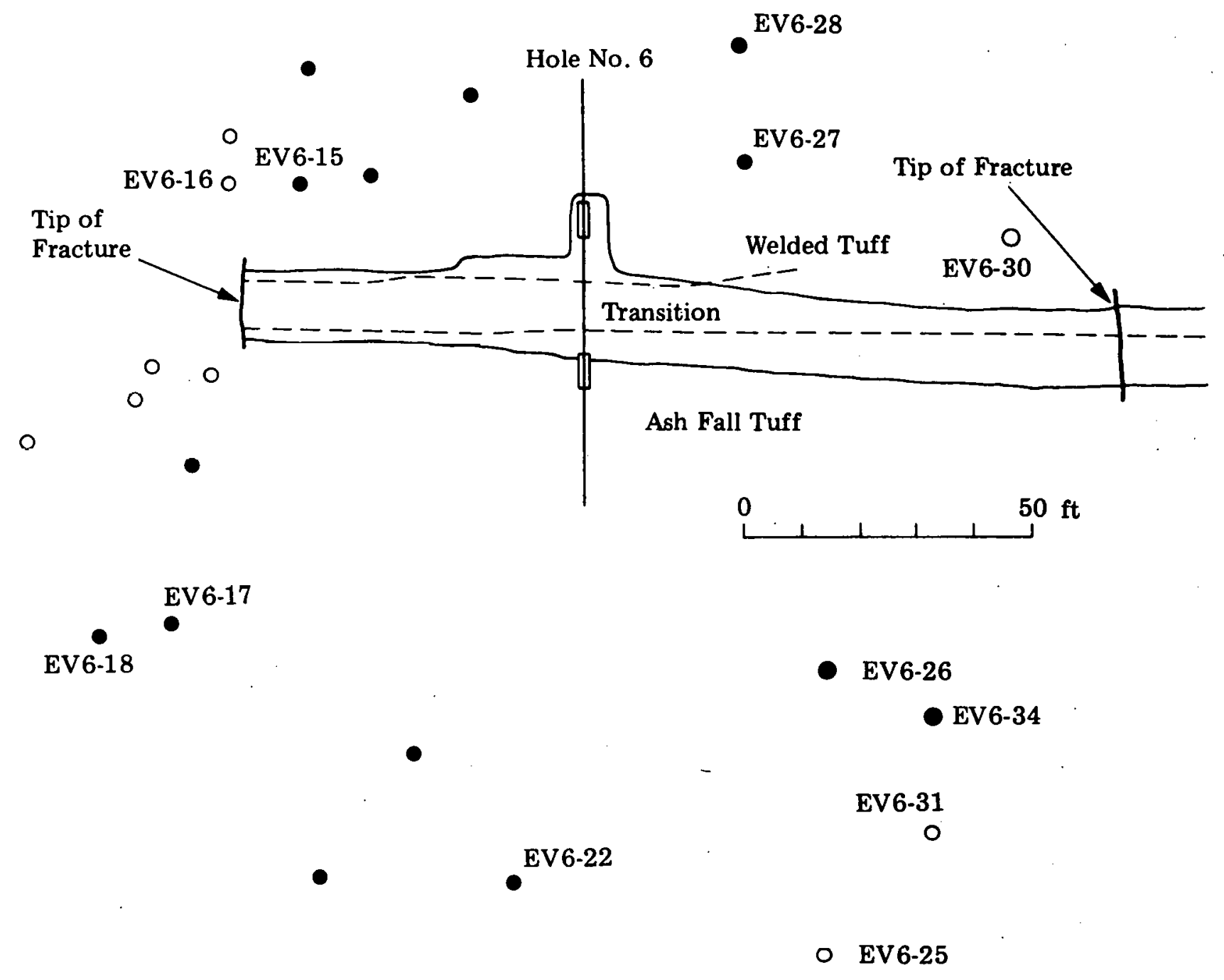

EV6-23

O EV6-24 $^{\text {EV }}$

Located Grout
No Grout

Figure 5-9 Experimental Coring Program of Hole No. 6 Fractures 


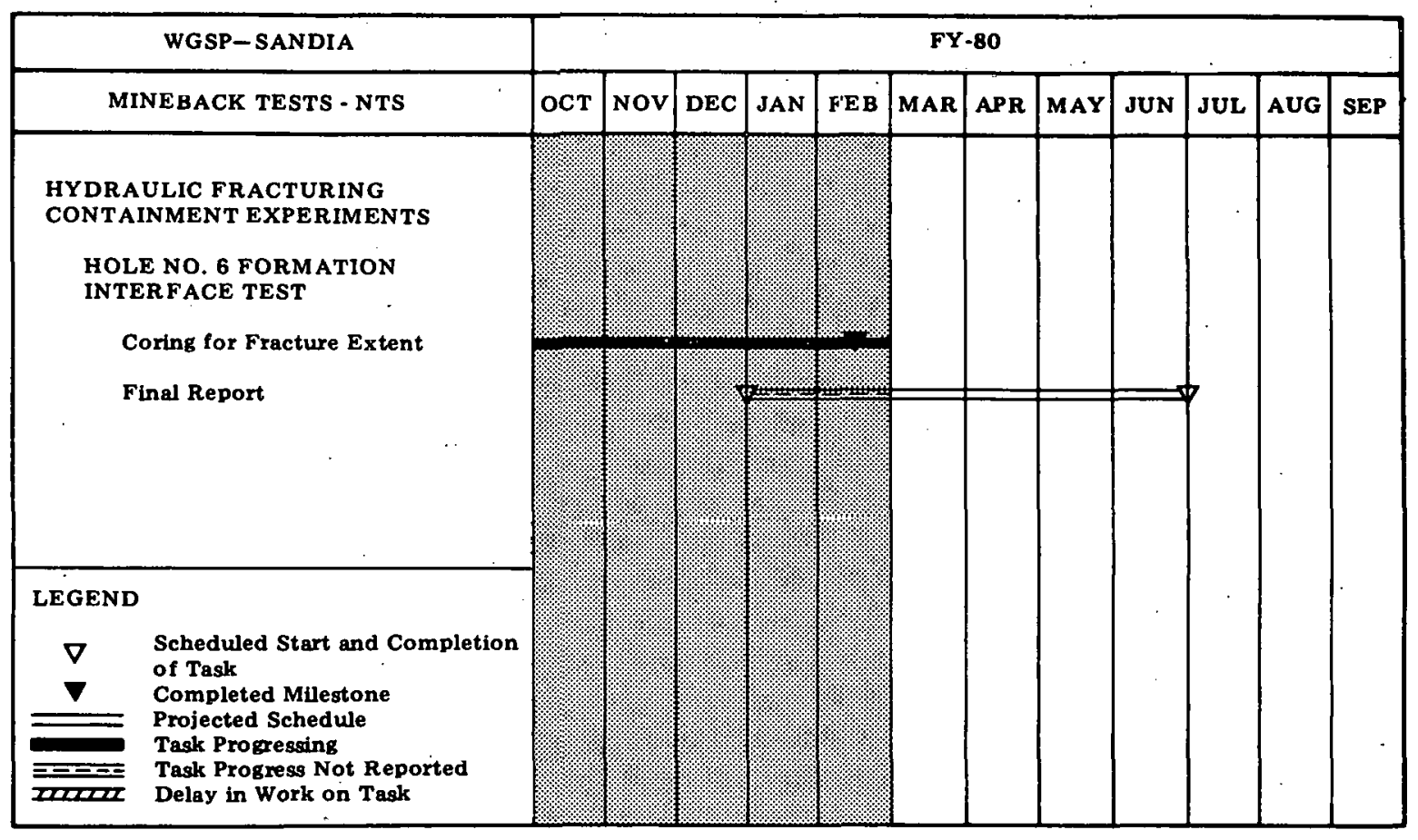

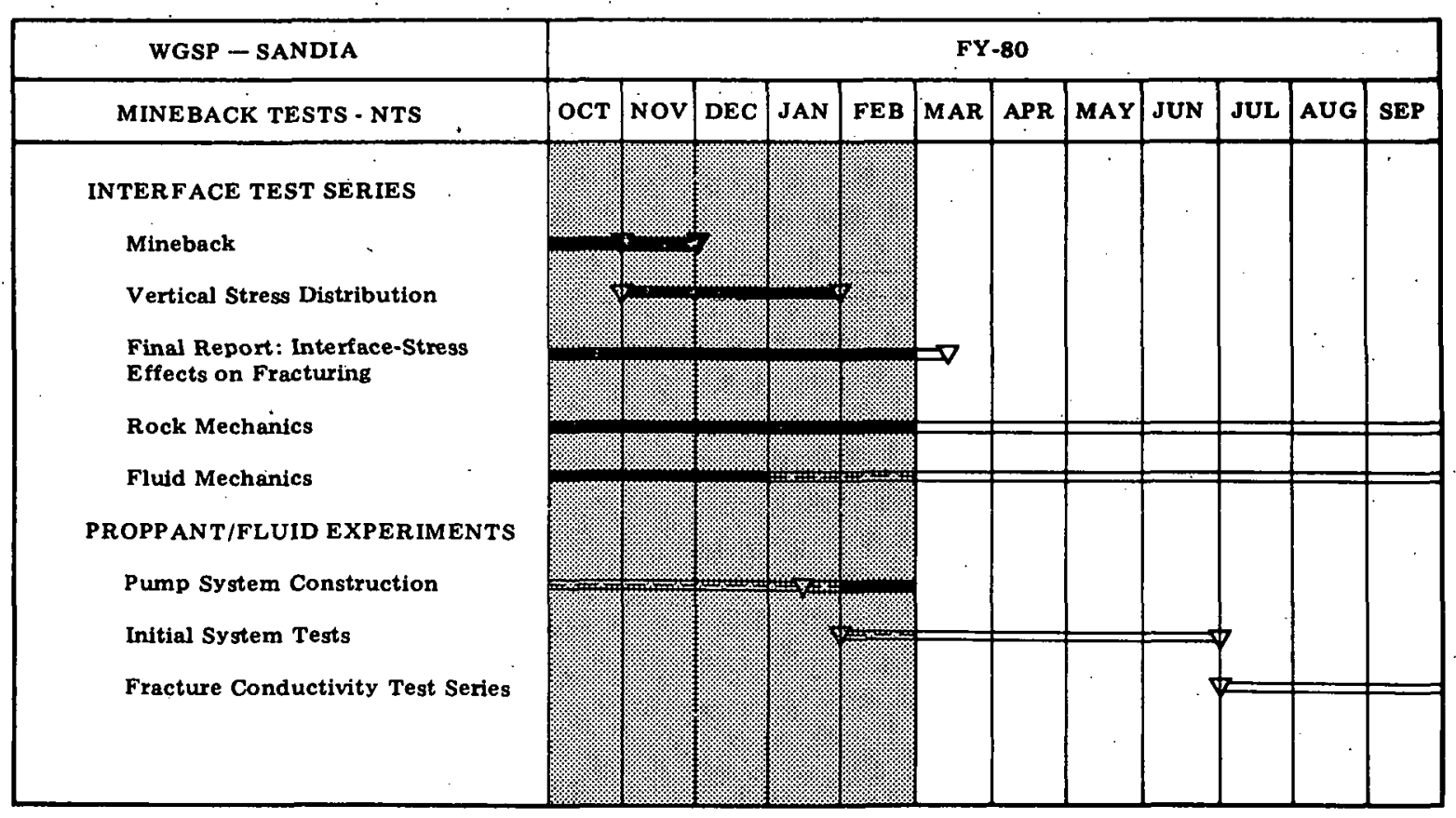

Figure 5-10 Milestone Chart-Sandia Mineback 
